\title{
Theoretical and computational models of biological ion channels
}

\author{
Benoît Roux*, Toby Allen, Simon Bernèche and Wonpil Im \\ Department of Biochemistry, Weill Medical College of Cornell University, I 300 York Avenue, New York, \\ NY 1002I, USA
}

\begin{abstract}
The goal of this review is to establish a broad and rigorous theoretical framework to describe ion permeation through biological channels. This framework is developed in the context of atomic models on the basis of the statistical mechanical projection-operator formalism of Mori and Zwanzig. The review is divided into two main parts. The first part introduces the fundamental concepts needed to construct a hierarchy of dynamical models at different level of approximation. In particular, the potential of mean force (PMF) as a configuration-dependent free energy is introduced, and its significance concerning equilibrium and non-equilibrium phenomena is discussed. In addition, fundamental aspects of membrane electrostatics, with a particular emphasis on the influence of the transmembrane potential, as well as important computational techniques for extracting essential information from all-atom molecular dynamics (MD) simulations are described and discussed. The first part of the review provides a theoretical formalism to 'translate' the information from the atomic structure into the familiar language of phenomenological models of ion permeation. The second part is aimed at reviewing and contrasting results obtained in recent computational studies of three very different channels: the gramicidin A (gA) channel, which is a narrow one-ion pore (at moderate concentration), the KcsA channel from Streptomyces lividans, which is a narrow multi-ion pore, and the outer membrane matrix porin $\mathrm{F}(\mathrm{OmpF})$ from Escherichia coli, which is a trimer of three $\beta$-barrel subunits each forming wide aqueous multi-ion pores. Comparison with experiments demonstrates that current computational models are approaching semi-quantitative accuracy and are able to provide significant insight into the microscopic mechanisms of ion conduction and selectivity. We conclude that all-atom MD with explicit water molecules can represent important structural features of complex biological channels accurately, including such features as the location of ion-binding sites along the permeation pathway. We finally discuss the broader issue of the validity of ion permeation models and an outlook to the future.
\end{abstract}

\section{Introduction 17}

\section{Dynamics of many-body systems 19}
2.I Effective dynamics of reduced systems 21
2.2 The constraint of thermodynamic equilibrium 24
2.3 Mean-field theories 25

\section{Solvation free energy and electrostatics 27}

3.1 Microscopic view of the Born model 27

3.2 Ion-lon interactions in bulk solution 29

* Author for correspondence.

Tel.: 212-746-6018; Fax: 212-746-4843; E-mail: benoit.roux@med.cornell.edu 
3.3 Continuum electrostatics and the PB equation 29

3.4 Limitations of continuum dielectric models 32

3.5 The dielectric barrier 33

3.6 The transmembrane potential and the PB-V equation 35

\section{Statistical mechanical equilibrium theory $\mathbf{4 0}$}

4.I Multi-ion PMF 40

4.2 Equilibrium probabilities of occupancy 43

4.3 Coupling to the membrane potential 44

4.4 lonic selectivity 48

4.5 Reduction to a one-dimensional (ID) free-energy profile 49

\section{From MD to $I-V$ : a practical guide $\mathbf{5 0}$}

5.I Extracting the essential ingredients from MD 5।

5.I.I Channel conductance from equilibrium and non-equilibrium MD 5 I

5.1.2 PMF techniques 52

5.1.3 Friction and diffusion coefficient techniques 53

5.I.4 About computational times 55

5.2 lon permeation models 56

5.2.I The ID-NP electrodiffusion theory 56

5.2.2 Discrete-state Markov chains 57

5.2.3 The GCMC/BD algorithm 58

5.2.4 PNP electrodiffusion theory 62

\section{Computational studies of ion channels $\mathbf{6 3}$}

6.1 Computational studies of gA 65

6.1.I Free-energy surface for $\mathrm{K}^{+}$permeation 66

6.1.2 Mean-force decomposition 69

6.1.3 Cation-binding sites 69

6.1.4 Channel conductance 70

6.1.5 Selectivity 72

6.2 Computational studies of KcsA 72

6.2.I Multi-ion free-energy surface and cation-binding sites 73

6.2.2 Channel conductance 74

6.2.3 Mechanism of ion conduction 77

6.2.4 Selectivity 78

6.3 Computational studies of OmpF 79

6.3.I The need to compare the different level of approximations 79

6.3.2 Equilibrium protein fluctuations and ion distribution 80

6.3.3 Non-equilibrium ion fluxes 80

6.3.4 Reversal potential and selectivity 84

6.4 Successes and limitations 87

6.4.I Channel structure 87

6.4.2 lon-binding sites 87

6.4.3 Ion conduction 88

6.4.4 Ion selectivity 89

7. Conclusion 90

\section{Acknowledgments 93}

\section{References 93}




\section{Introduction}

Ever since the early days of electrophysiology, the development of theoretical models of ion channels has contributed to a better understanding and interpretation of experimental data (Hille, 2001). Although the recent progress in the determination of three-dimensional (3D) structures of biological ion channels provides a wealth of information (Ketchem et al. 1997; Chang et al. 1998; Doyle et al. 1998; Schirmer, 1998; Morais-Cabral et al. 2001; Wang et al. 2001; Zhou et al. 2001a,b), theoretical considerations are necessary for elucidating the (hidden) microscopic factors governing ion conduction and selectivity at the atomic level. The combination of atomic resolution structures plus highly sophisticated computational approaches offers the possibility of a virtual route for interpreting and relating a channel structure to its function. But to achieve this, it is necessary to establish a rigorous language enabling us to 'translate' the information from the atomic structure into the observed function. Addressing questions about ion permeation and selectivity through molecular pores in a meaningful way is, however, difficult because it requires an ability to calculate ion fluxes with quantitative accuracy. At the present time, these questions cannot be addressed with a unique approach and a wide variety of computational methods contribute to refine our understanding of ion channels (Kuyucak et al. 2001; Tieleman et al. 2001b; Chung \& Kuyucak, 2002; Roux, 2002b).

Arguably, all-atom molecular dynamics (MD) represents the most detailed approach to study complex biomolecular systems. It consists in constructing an atomic model of the macromolecular system, representing the microscopic forces with a potential function, and integrating Newton's classical equation ' $F=M A$ ' to generate a trajectory (for a recent review of simulation methods, see chapters 1-4 in Becker et al. 2001). The result is literally a 'simulation' of the dynamical motions of all the atoms as a function of time. With the availability of potential energy functions for proteins and lipids, as well as fast and reliable numerical algorithms, current MD methodologies have reached the point where one can generate trajectories of realistic atomic models of complex biological-channel membrane systems. In recent years, MD simulations with explicit membranes have been used extensively to study an increasingly large number of ion channels; gramicidin (gA) (Woolf \& Roux, 1997; Chiu et al. 1999a; Roux, 2002a; Allen et al. 2003a, b), alamethicin (Alm) (Tieleman et al. 1999, 2001a; Smith \& Sansom, 2002), the transmembrane domain of the influenza virus (M2) (Forrest et al. 2000; Zhong et al. 2000), OmpF porin (Suenaga et al. 1998; Tieleman \& Berendsen, 1998; Im \& Roux, 2002a), the mechanosensitive channel MscL (Elmore \& Dougherty, 2001; Gullingsrud et al. 2001; Colombo et al. 2003; Gullingsrud \& Schulten, 2003), and $\mathrm{K}^{+}$channels (Allen et al. 1999, 2000; Guidoni et al. 1999, 2000; Åqvist \& Luzhkov, 2000; Bernèche \& Roux, 2000, 2001; Capener et al. 2000; Luzhkov \& Åqvist, 2000, 2001a, b; Shrivastava \& Sansom, 2000; Biggin et al. 2001; Crouzy et al. 2001; Burykin et al. 2002, 2003).

Simple MD trajectories, however, are somewhat limited in their ability to quantitatively characterize complex biomolecular systems. Nonetheless their scope can be extended considerably with advanced computational techniques that will be the focus of this review, such as

Abbreviations: Alm, alamethicin; BD, Brownian dynamics; DPPC, dipalmitoyl phosphatidylcholine; DMPC, dimyristoyl phosphatidylcholine; EMF, electromotive force; ERT, Eyring Rate Theory; FEP, free energy perturbation; gA, gramicidin A; GCMC, Grand Canonical Monte Carlo; GLE, generalized Langevin equation; HNC, hypernetted chain; LE, Langevin equation; MD, molecular dynamics; MSA, mean-spherical approximation; MSD, mean square displacement; NMR, nuclear magnetic resonance; PB, Poisson-Boltzmann; PB-V, Poisson-Boltzmann voltage; PMF, potential of mean force; PNP, Poisson-Nernst-Planck; PY, Percus-Yevick; WHAM, weighted histogram analysis method. 
free energy perturbation (FEP) (Allen et al. 1999, 2000; Åqvist \& Luzhkov, 2000; Luzhkov \& Åqvist, 2000, 2001a; Bernèche \& Roux, 2001) and umbrella sampling (Bernèche \& Roux, 2001; Crouzy et al. 2001; Allen et al. 2003b), or by the introduction of external forces applied on the system (Suenaga et al. 1998; Crozier et al. 2001a, b; Gullingsrud et al. 2001; Tieleman et al. 2001a; Yang et al. 2003). FEP calculations use simulations generated with an unphysically altered potential function; thermodynamic integration is then performed to compute the free-energy changes between different states of the system. Umbrella sampling calculations use simulations generated in the presence of an imposed biasing potential to enhance configurational sampling; the effect of this bias is then removed in post-analysis to compute the unbiased potential of mean force (PMF) of the system (Becker et al. 2001). It is also possible to monitor the dynamical motions in the presence of imposed external forces to reproduce some aspect of the environment such as membrane surface tension (Gullingsrud et al. 2001), or transmembrane voltage (Suenaga et al. 1998; Crozier et al. 2001a, b; Tieleman et al. 2001a; Yang et al. 2003). The latter is of particular importance for simulations of ion channels.

In addition, approaches that are simpler and computationally less expensive than all-atom MD are very important tools in studies of ion channels. In particular, macroscopic continuum electrostatic calculations, in which the polar solvent is represented as a structureless dielectric medium, can help reveal the dominant energetic factors related to ion permeation and, thus, serve to illustrate fundamental principles in a particularly clear fashion (Roux, 1999a; Roux et al. 2000). Brownian dynamics (BD), which consists in integrating stochastic equation of motions describing the displacement of the ions with some effective potential function, generally calculated on the basis of a continuum electrostatic approximation (Cooper et al. 1985; Chung et al. 1998), is also an attractive computational approach for simulating the permeation process over long time-scales without having to treat all the solvent molecules explicitly (Schirmer \& Phale, 1999; Im et al. 2000; Allen \& Chung, 2001; Im \& Roux, 2001; Mashl et al. 2001; Phale et al. 2001; Burykin et al. 2002, 2003). Lastly, there are continuum electrodiffusion theories, such as Poisson-Nernst-Planck (PNP), which attempt to represent average ion fluxes directly in terms of concentration gradient and average electric field (Onsager, 1926, 1927; Schuss et al. 2001).

Which approach is best to use may depend on the microscopic detail of a particular channel, the specific questions being asked, and the available computational resources. There is, however, a lack of consensus concerning the relative merits and limitations of the various approaches, and their range of validity is often a matter of debate (Levitt, 1999; McClesky, 1999; Miller, 1999; Nonner et al. 1999; Roux, 1999b). Biological ion channels are macromolecular many-body systems. From this point of view, it is not surprising that such complexity calls for a hierarchy of inter-related computational approaches, corresponding to different levels of approximations. The existence of so many different approaches does, however, generate some confusion. Sometimes, it even becomes difficult to distinguish important fundamental conceptual differences from simple technical variations in computational schemes and procedures. Theoretical models, at any level, are approximations and have intrinsic limitations. The result from any computation is not meaningful if the underlying theory is taken beyond its range of validity. Comparing and relating the different approaches is difficult, however, partly because of the lack of a unified formal language.

One of the main goals of this review is to formulate a broad and general theoretical framework to describe ion permeation. It is our hope that such a framework will help clarify the correspondence between the various approaches that currently are used in computational studies of ion channels. The review is divided into two main parts. The first part (sections 2-5) is mostly 
concerned with elaborating and establishing the fundamental concepts, laying the foundation for a unified discussion of the various approaches used in theoretical studies of ion channels. The second part (sections 6-7) is aimed at reviewing and contrasting the most important results obtained in recent theoretical studies of three very different channels: the gA channel, the KcsA $\mathrm{K}^{+}$channel from Streptomyces lividans, and the outer membrane matrix porin $\mathrm{F}(\mathrm{OmpF})$ from Escherichia coli. It is our hope that the basic elements discussed in the first part of the review provide a rigorous theoretical framework to describe ion permeation through biological channels, while the practical cases discussed in the second part can help illustrate the most important concepts.

In Section 2, we first review and highlight some essential concepts concerning the dynamics of many-body systems and the theoretical approaches that enable one to construct a hierarchy of dynamical models, going from all-atom MD to BD. In particular, the PMF as a configurationdependent free energy will be first introduced, and its significance regarding equilibrium and non-equilibrium phenomena will be discussed. In Section 3, we briefly review the main elements of solvation free energy in liquid water, including a discussion of the Born model and ion-ion interactions at the microscopic level. Then, we review some basic concepts of membrane electrostatics, with a particular emphasis on the influence of the transmembrane potential. In Section 4, a rigorous statistical mechanical theory of the equilibrium properties of ion channels is formulated and the concept of multi-ion PMF is established from first principles. Finally, the most important computational approaches for extracting essential ingredients from all-atom MD to incorporate those into framework theories for simulating non-equilibrium ion flow are discussed in Section 5. In particular, a number of useful computational techniques for calculating the PMF as well as the diffusion coefficient on the basis of all-atom MD simulations are briefly reviewed, and important approaches such as Grand Canonical Monte Carlo (GCMC)/BD simulations and PNP are exposed. The formal and technical part of the review (sections 2-5) is then followed, in Section 6, by an overview of the results obtained in theoretical studies of the gA channel, which is a narrow 1-ion pore (at moderately low ion concentrations), the KcsA channel, which is a narrow multi-ion pore, and OmpF porin, which is a trimer of three $\beta$-barrel subunits each forming wide aqueous multi-ion pores. The molecular structure of these channels is shown in Fig. 1. Contrasting the properties of these three very different channels helps illustrate important principles. In particular, the gA and KcsA channels exemplify important characteristics of a narrow pore with tight interactions between the permeating ion and the protein, while KcsA and $\mathrm{OmpF}$ reveal the role of ion-ion interactions in multi-ion channels. Furthermore, OmpF illustrates the influence of counterions and electrostatic screening in a wide aqueous pore. The main strength and limitations of current models are discussed in. The review is concluded in Section 7 with a broad discussion of the validity of ion permeation models and an outlook to the future.

\section{Dynamics of many-body systems}

In $\mathrm{MD}$, a trajectory of the system is generated by integrating numerically Newton's classical equation of motion,

$m_{i} \ddot{\mathbf{r}}_{i}=\mathbf{F}_{i}$,

where $m_{i}, \mathbf{r}_{i}$, and $\ddot{\mathbf{r}}_{i}$ are the mass, position, and acceleration of the $i$ th atom respectively, and the microscopic force acting on atom $i$ is

$\mathbf{F}_{i}=-\nabla_{i} U$, 
(a)
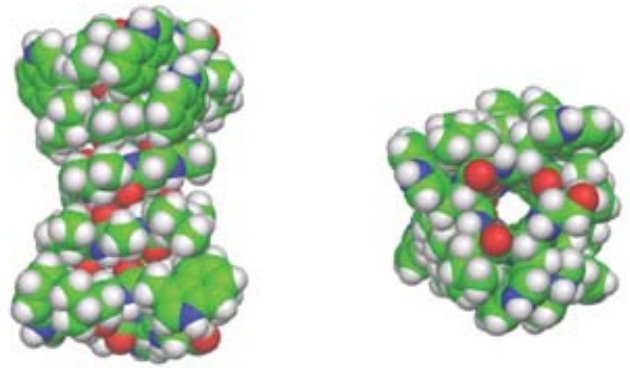

(b)
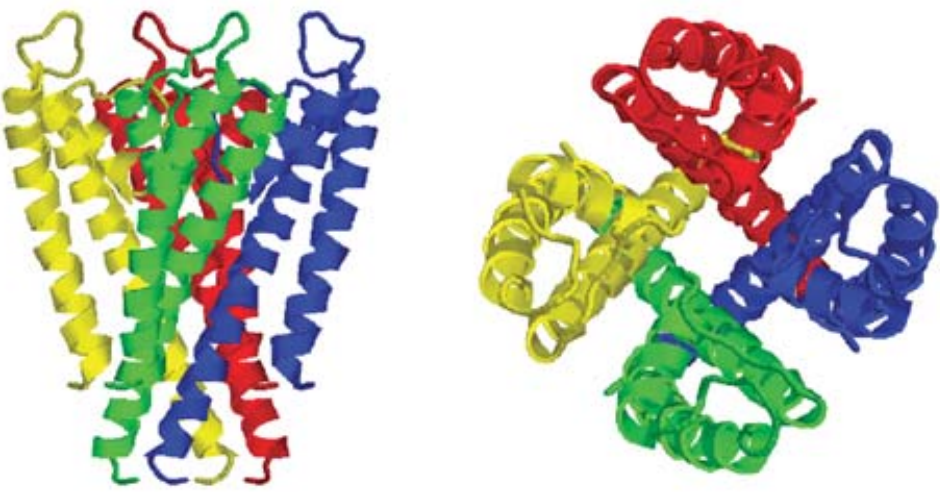

(c)
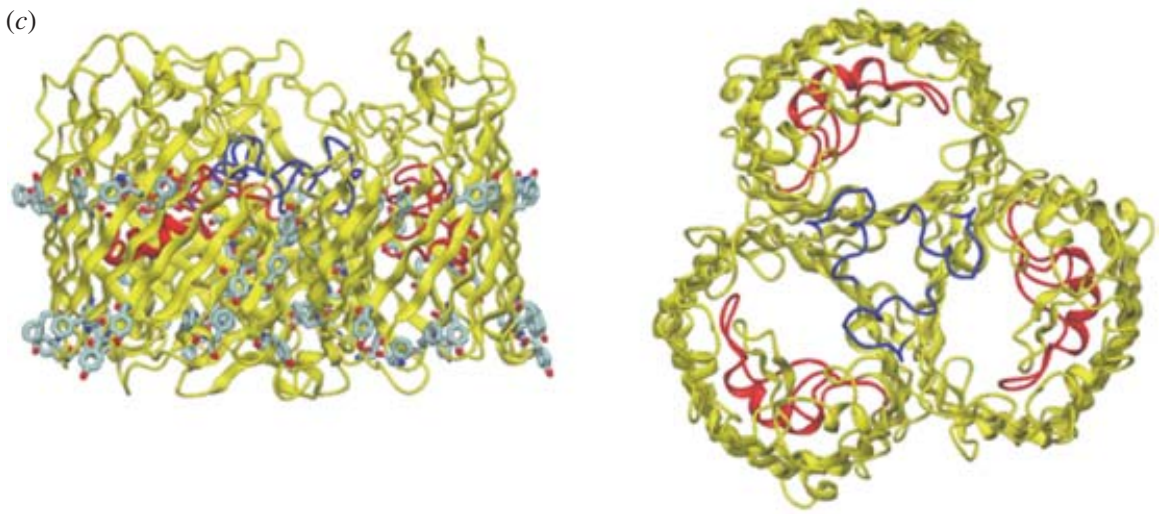

Fig. 1. (a) Gramicidin channel from Townsley et al. (2001). (b) KcsA channel from Zhou et al. (2001b). (c) OmpF porin from Cowan et al. (1992). The three channels are not drawn to scale.

where $U$ is the potential function of the system. For computational efficiency, the potential function used in biomolecular simulations are normally constructed from simple analytical functions (Becker et al. 2001), though it is important to keep in mind that $U$ is meant to represent the quantum-mechanical Born-Oppenheimer energy surface of the molecular system. All-atom MD provides a detailed structural and dynamical atomic model of the biomolecular system of interest. Some examples of ion channel systems simulated by MD are shown in Fig. 2. In those studies, the microscopic system includes explicitly the water molecules, the ions, as well as the phospholipid membrane bilayer. Typically, such all-atom microscopic models may include several tens of thousands of particles. Once such a system has been properly equilibrated, average properties can be calculated directly from the trajectory of the atoms. 
(a)

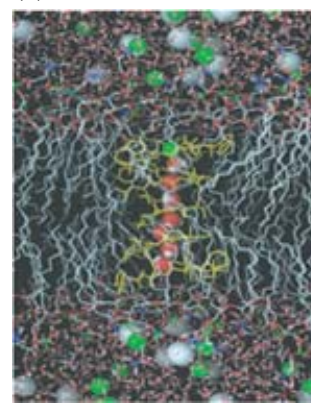

(b)

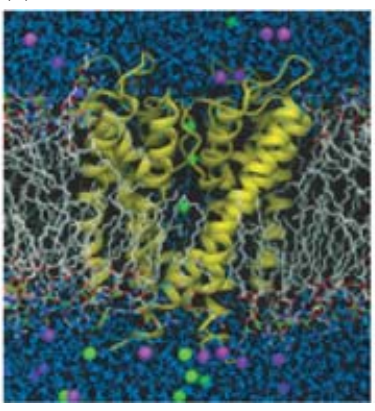

(c)

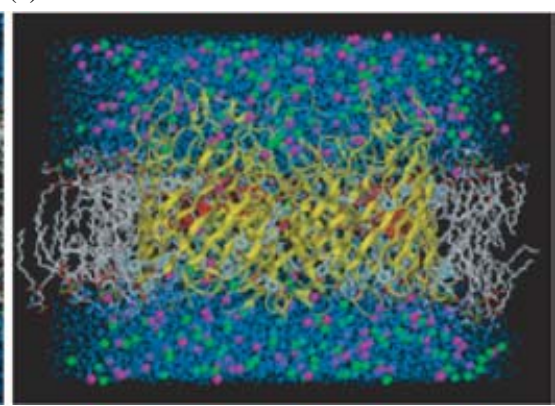

Fig. 2. Molecular graphics representation of the atomic models simulated with all-atom molecular dynamics (a) Gramicidin A in a DMPC bilayer (Allen et al. 2003a). (b) KcsA K $\mathrm{K}^{+}$channel in DPPC phosphilipid membrane bathed by a $150 \mathrm{~mm} \mathrm{KCl}$ aqueous salt solution. (c) Molecular graphics view of OmpF porin embedded in a DMPC membrane in an aqueous solution of $1 \mathrm{M}[\mathrm{KCl}]$ salt (the $\mathrm{K}^{+}$ions are magenta and the $\mathrm{Cl}^{-}$ions are green). For clarity, some lipid molecules were removed from the front view. The three channels are not drawn to scale.

The most striking drawback of all-atom MD is that the trajectory of a large number of atoms must be calculated, although only a small number among them (e.g. the permeating ions in the channel) might be truly relevant to understand the function that one wishes to investigate. It is often tempting to try to decrease the size of the simulated system to 'bare essentials', but this may be at the risk of altering important microscopic factors, and ultimately undermining the value and significance of an atomic model. Unavoidably, a large amount of what is being simulated is not necessarily of immediate interest in such 'brute force' calculations, but it is essential in order to have a valuable microscopic model. An attractive approach would be to focus only on the dynamics of the most relevant variables, i.e. the ions in the neighborhood of the channel, and yet retain the ability to represent all their microscopic properties (structural and dynamical) correctly. Certainly, such an approach might serve as a guide to design useful approximations and plan efficient computational strategies aimed at reducing the computer time required to simulate ion permeation. Gains in computational efficiency is not, however, the only motivation for adopting this perspective on ion permeation. After all, there is a steady increase in computer power, and relatively soon, one should be able to simulate ion permeation through biological channels directly with all-atom MD. Nevertheless, even when the computational time will no longer be a limiting factor, it would be difficult to extract fundamental principles and develop a rigorous perspective on ion permeation solely based on atomic trajectories. Therefore, the ultimate value of formulating a dynamical theory focused on the most relevant degrees of freedom should be to provide a rigorous conceptual framework for representing the wide range of equilibrium and non-equilibrium phenomena taking place in the complex molecular system that are ion channels.

\section{I Effective dynamics of reduced systems}

The problem of representing the dynamics of a reduced set of degrees of freedom, itself part of a large complex system, is a subject that has been extensively explored in statistical mechanical theories over the last 50 years. One of the most powerful approaches to tackle this problem is the projection operator theory, which was originally introduced by Zwanzig (1961) and Mori (1965). The general idea consists in 'projecting out' uninteresting degrees of freedom, in order to 
develop an effective (but physically correct) dynamical scheme for representing the time evolution of the most 'relevant' degrees of freedom realistically (Zwanzig, 2001). Typically, the subset of coordinates that are treated explicitly evolves according to some effective, nondeterministic (stochastic) dynamics, which incorporates the influence of the rest of the system implicitly. In contrast, the classical MD trajectory of the all-atom system, which is calculated according to Newton's classical law of motion Eq. (1), is deterministic (because all the degrees of freedom are included explicitly). Within certain limits discussed below, the projection operator theory is formally exact. It provides a rigorous framework to construct approximate models of ion permeation in a simple and physically appealing fashion.

To illustrate the main principles involved in the effective dynamics of reduced systems, we consider a dense liquid solution. Let us choose the position of a subset of 'tagged' particles, $\left\{\mathbf{r}_{1}, \mathbf{r}_{2}, \ldots\right\}$, as the most 'relevant' coordinates. Based on projection operator theory, the dynamics of these particles evolves according to a generalized Langevin Equation (GLE) (Zwanzig, 1965; Mazur \& Oppenheim, 1970, 1982; Adelman, 1980)

$m_{i} \ddot{\mathbf{r}}_{i}(t)=\mathcal{F}_{i}-\int_{0}^{t} \mathrm{~d} t^{\prime} M_{i}\left(t-t^{\prime}\right) \dot{\mathbf{r}}_{i}\left(t^{\prime}\right)+\mathbf{f}_{i}(t)$.

The GLE has some similarity with Newton's classical equation of motion, Eq. (1), but there are also some important differences. In particular, the force acting on the $i$ th particle is not calculated from the potential energy $U$, but from the effective potential $\mathcal{W}$,

$\mathcal{F}_{i}\left(\mathbf{r}_{1}, \mathbf{r}_{2}, \ldots\right)=-\nabla_{i} \mathcal{W}\left(\mathbf{r}_{1}, \mathbf{r}_{2}, \ldots\right)$.

While the potential energy $U$ depends on the position of all the atoms in the system, it should be stressed that the effective potential $\mathcal{W}$, and the effective force $\mathcal{F}_{i}$, depend only on the subset of relevant coordinates $\left\{\mathbf{r}_{1}, \mathbf{r}_{2}, \ldots\right\}$. The significance of $\mathcal{W}$ will be examined further below. There are also two additional terms, not normally present in Newton's equation. First, there is a convolution in the time domain of the particle's velocity with a memory function, $M(t)$. On average, this term gives rise to a dragging force opposing the movement of the particles. Lastly, there is also a time-dependent external force, $\mathbf{f}_{i}(t)$, acting on the reduced system. It can be shown that, $\mathbf{f}_{i}(t)$ is completely uncorrelated with the time-course of the set of variables $\left\{\mathbf{r}_{1}(t), \mathbf{r}_{2}(t), \ldots\right\}$, i.e. it behaves as an external stochastic random force. Two important properties of this random force are $\left\langle\mathbf{f}_{i}(t)\right\rangle=0$ and $\left\langle\mathbf{f}_{i}(t) \cdot \mathbf{f}_{i}(0)\right\rangle=3 k_{\mathrm{B}} T M_{i}(t)$, where $k_{\mathrm{B}}$ is Boltzmann's constant and $T$ is the temperature; the amplitude of $\mathbf{f}_{i}$ is typically assumed to be Gaussian-distributed (Zwanzig, 1965; Mazur \& Oppenheim, 1970, 1982; Adelman, 1980). The relationship between the random force and the memory function is called the 'fluctuation-dissipation theorem' (Kubo, 1966). Both the dissipative force arising from the dragging and the external random time-dependent force incorporate the influence of impacts and collisions with those other particles that are not treated explicitly.

A guiding rule to choose the set of relevant variables is that it should explicitly include all the slowest processes that could possibly take place in the system. In dense molecular bulk media, the memory function associated with a tagged particle typically decays very rapidly compared to all other time-scales in the system (Mazur \& Oppenheim, 1970, 1982; Adelman, 1980). Because proteins are fairly rigid, the memory function for an ion inside a membrane channel may be expected to decay quite rapidly. An example of a memory function of an ion in a model of the gA channel is shown in Fig. 3. It decays to zero in $\sim 0.5$ ps. The Markov assumption consists in making the approximation that the memory function decays instantaneously compared to all 


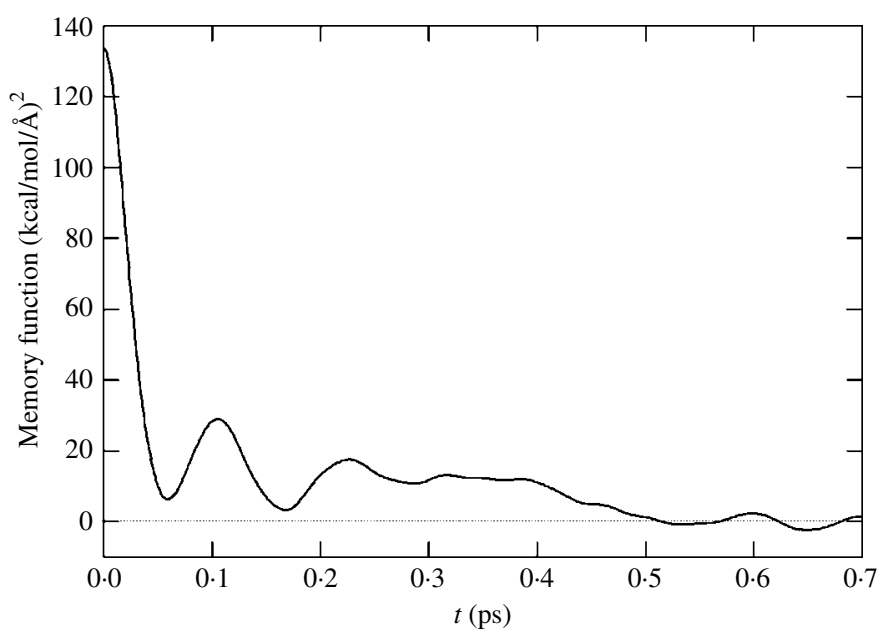

Fig. 3. Memory function of a $\mathrm{Na}^{+}$ion in a model of the gramicidin A channel. The memory function was calculated as a force-force correlation function (Roux \& Karplus, 1991a).

relevant time-scales,

$$
\begin{aligned}
-\int_{0}^{t} \mathrm{~d} t^{\prime} M_{i}\left(t-t^{\prime}\right) \dot{\mathbf{r}}_{i}\left(t^{\prime}\right) & \approx-\dot{\mathbf{r}}_{i}(t) \int_{0}^{\infty} \mathrm{d} t M_{i}\left(t-t^{\prime}\right) \\
& \approx-\gamma_{i} \dot{\mathbf{r}}_{i}(t),
\end{aligned}
$$

where $\gamma_{i}$ is the friction coefficient

$$
\begin{aligned}
\gamma_{i} & =\int_{0}^{\infty} \mathrm{d} t M_{i}(t) \\
& =\frac{1}{3 k_{\mathrm{B}} T} \int_{0}^{\infty} \mathrm{d} t\left\langle\mathbf{f}_{i}(t) \cdot \mathbf{f}_{i}(0)\right\rangle .
\end{aligned}
$$

This assumption leads to the classical Langevin Equation (LE) (Chandrasekar, 1943; McQuarrie, 1976)

$m_{i} \ddot{\mathbf{r}}_{i}(t)=\mathcal{F}_{i}-\gamma_{i} \dot{\mathbf{r}}_{i}(t)+\mathbf{f}_{i}(t)$,

where $\mathbf{f}_{i}(t)$ is a random Gaussian force obeying the fluctuation-dissipation theorem $\left\langle\mathbf{f}_{i}(t) \cdot \mathbf{f}_{i}(0)\right\rangle$ $=6 \gamma_{i} k_{\mathrm{B}} T \delta(t)$. If the friction coefficient $\gamma_{i}$ is large, the motions become overdamped and the inertial term, $\mathrm{m}_{\mathbf{i}} \ddot{\mathbf{i}}_{i}(t)$, may be neglected. This approximation yields the BD equation (Chandrasekar, 1943)

$\dot{\mathbf{r}}_{i}(t)=\frac{D_{i}}{k_{\mathrm{B}} T} \mathcal{F}_{i}+\zeta_{i}(t)$

where $\mathrm{D}_{i}=k_{\mathrm{B}} T / \gamma_{i}$ is the diffusion coefficient of the tagged particles, and $\zeta_{i}(t)$ is a random Gaussian noise with $\left\langle\xi_{i}(t) \cdot \zeta_{i}(0)\right\rangle=6 D_{i} \delta(t)$. It may be noted that the mass of the diffusing particle does not appear in the non-inertial high friction limit. This regime, which is sometimes referred to as 'low Reynolds number' (Purcell, 1977), is somewhat counter-intuitive compared to our own experience in the macroscopic world. For example, ions never undergo large 'ballistic' displacements in a dense liquid or inside a channel. 


\subsection{The constraint of thermodynamic equilibrium}

A common ingredient in all the reduced dynamical models described above is the effective potential $\mathcal{W}$, which is used to compute the effective forces $\mathcal{F}_{i}$, in Eq. (4). Remarkably, it can be shown that the equilibrium properties of all such effective dynamical system are independent of the character of the dissipative and fluctuating forces included in the model (as long as these forces correctly obey the fluctuation-dissipation theorem). In spite of important differences in the time-dependent properties displayed by the various stochastic effective dynamics (GLE, LE, or $\mathrm{BD})$, ultimately, the probability distribution of the coordinates $\left\{\mathbf{r}_{1}, \mathbf{r}_{2}, \ldots\right\}$ is given by

$P_{\text {eq }}\left(\mathbf{r}_{1}, \mathbf{r}_{2}, \ldots\right) \propto \mathrm{e}^{-\mathcal{W}\left(\mathbf{r}_{1}, \mathbf{r}_{2}, \ldots\right) / k_{\mathrm{B}} T}$

(note that the temperature $T$ appearing here entered the stochastic dynamics only via the relation between the amplitude of the dissipative and fluctuating forces). For example, the timedependent probability distribution associated with the stochastic trajectories generated from BD as in Eq. (8) evolves according to the multi-dimensional Smoluchowski diffusion equation (Smoluchowski, 1916; Chandrasekar, 1943),

$\frac{\partial P\left(\mathbf{r}_{1}, \mathbf{r}_{2}, \ldots, t\right)}{\partial t}=-\sum_{i} \boldsymbol{\nabla}_{i} \cdot\left[-D_{i} \boldsymbol{\nabla}_{i} P\left(\mathbf{r}_{1}, \mathbf{r}_{2}, \ldots, t\right)-\frac{D_{i}}{k_{\mathrm{B}} T} \boldsymbol{\nabla}_{i} \mathcal{W}\left(\mathbf{r}_{1}, \mathbf{r}_{2}, \ldots\right) P\left(\mathbf{r}_{1}, \mathbf{r}_{2}, \ldots, t\right)\right]$

and it is easy to show that the equilibrium distribution $P_{\text {eq }}$ in Eq. (9) yields a time-independent solution to Eq. (10).

$\mathcal{W}$ determines all the underlying equilibrium structural properties arising from the stochastic dynamics of the reduced system. For this reason, it is the most crucial ingredient in constructing meaningful models of reduced systems (GLE, LE or BD). Therefore, an important constraint on $\mathcal{W}$ is that averages resulting from the stochastic dynamics of the reduced effective system be equal to the average resulting from the all-atom MD simulation, for any observable quantity $Q$, depending on the configuration of the tagged particles. But is this even possible in principle? Let us examine this question in more detail. In the reduced system, such an average is expressed as,

$$
\langle Q\rangle_{(\mathrm{red})}=\frac{\int \mathrm{d} \mathbf{r}_{1} \int \mathrm{d} \mathbf{r}_{2} \cdots Q\left(\mathbf{r}_{1}, \mathbf{r}_{2}, \ldots\right) \mathrm{e}^{-\mathcal{W}\left(\mathbf{r}_{1}, \mathbf{r}_{2}, \ldots\right) / k_{\mathrm{B}} T}}{\int \mathrm{d} \mathbf{r}_{1} \int \mathrm{d} \mathbf{r}_{2} \cdots \mathrm{e}^{-\mathcal{W}\left(\mathbf{r}_{1}, \mathbf{r}_{2}, \ldots\right) / k_{\mathrm{B}} T}},
$$

and involves only the relevant coordinates $\left\{\mathbf{r}_{1}, \mathbf{r}_{2}, \ldots\right\}$. In contrast, the corresponding average in the all-atom system is

$$
\langle Q\rangle_{(\text {all })}=\frac{\int \mathrm{d} \mathbf{r}_{1} \int \mathrm{d} \mathbf{r}_{2} \cdots \int \mathrm{d} \mathbf{X} Q\left(\mathbf{r}_{1}, \mathbf{r}_{2}, \ldots\right) \mathrm{e}^{-U\left(\mathbf{r}_{1}, \mathbf{r}_{2}, \ldots, \mathbf{X}\right) / k_{\mathrm{B}} T}}{\int \mathrm{d} \mathbf{r}_{1} \int \mathrm{d} \mathbf{r}_{2} \cdots \int \mathrm{d} \mathbf{X} \mathrm{e}^{-U\left(\mathbf{r}_{1}, \mathbf{r}_{2}, \ldots, \mathbf{X}\right) / k_{\mathrm{B}} T}},
$$

where $\mathbf{X}$ represents all the remaining degrees of freedom in the system others than the coordinates $\left\{\mathbf{r}_{1}, \mathbf{r}_{2}, \ldots\right\}$. For example, $\mathbf{X}$ could include all the solvent molecules, the protein channel and the phospholipid bilayer. The two expressions in Eqs. (11) and (12) for the average will be exactly the same if, and only if, $\mathcal{W}$ is constructed according to

$\mathrm{e}^{-\mathcal{W}\left(\mathbf{r}_{1}, \mathbf{r}_{2}, \ldots\right) / k_{\mathrm{B}} T}=C \int \mathrm{d} \mathbf{X} \mathrm{e}^{-U\left(\mathbf{r}_{1}, \mathbf{r}_{2}, \ldots, \mathbf{X}\right) / k_{\mathrm{B}} T}$,

where $C$ is an arbitrary constant. Obviously, $\mathcal{W}$ does not correspond to some average of the microscopic potential, i.e. $\mathcal{W} \neq\langle U\rangle$. An important property of $\mathcal{W}$ and its relationship to the 
all-atom system is highlighted by considering the derivative with respect to a coordinate $\mathbf{r}_{i}$,

$$
\begin{aligned}
\mathcal{F}_{i}\left(\mathbf{r}_{1}, \mathbf{r}_{2}, \ldots\right) & =-\nabla_{i} \mathcal{W}\left(\mathbf{r}_{1}, \mathbf{r}_{2}, \ldots\right) \\
& =\frac{\int \mathrm{d} \mathbf{X}-\nabla_{i} U\left(\mathbf{r}_{1}, \mathbf{r}_{2}, \ldots, \mathbf{X}\right) \mathrm{e}^{-U\left(\mathbf{r}_{1}, \mathbf{r}_{2}, \ldots, \mathbf{X}\right) / k_{\mathrm{B}} T}}{\int \mathrm{d} \mathbf{X} \mathrm{e}^{-U\left(\mathbf{r}_{1}, \mathbf{r}_{2}, \ldots, \mathbf{X}\right) / k_{\mathrm{B}} T}} \\
& =-\left\langle\boldsymbol{\nabla}_{i} U\right\rangle_{\left(\mathbf{r}_{1}, \mathbf{r}_{2}, \ldots\right)} \\
& =\left\langle\mathbf{F}_{i}\right\rangle_{\left(\mathbf{r}_{1}, \mathbf{r}_{2}, \ldots\right)} .
\end{aligned}
$$

This demonstrates that the effective force calculated from the gradient of $\mathcal{W}$ evaluated at $\left\{\mathbf{r}_{1}, \mathbf{r}_{2}, \ldots\right\}$ is equal to the total average force acting on the tagged particles when they are in this configuration in the all-atom system, i.e. the effect of all other atoms is included implicitly in $\mathcal{W}$ ! Because the effective potential ' $\mathcal{W}$ ' corresponds to a reversible thermodynamic 'work' done by the average force, it is called the PMF. The concept of PMF was first introduced by Kirkwood (1935) to describe distribution functions in liquids. The structure of $\mathcal{W}$ in Eq. (13) is similar to that of a configuration-dependent free energy. This is expressed in a particularly clear fashion by introducing a dimensionless thermodynamic coupling parameter $\lambda$, varying between 0 and 1 , such that $U(\lambda=1)$ is the normal potential energy and $U(\lambda=0)$ is the potential energy of a reference system in which all interactions involving the subset of tagged particles has been 'turned off' (Kirkwood, 1935). According to Eq. (13), the PMF can be written as the ratio of configurational integrals,

$\mathrm{e}^{-\mathcal{W}\left(\mathbf{r}_{1}, \mathbf{r}_{2}, \ldots\right) / k_{\mathrm{B}} T}=\frac{\int \mathrm{d} \mathbf{X} \mathrm{e}^{-U\left(\mathbf{r}_{1}, \mathbf{r}_{2}, \ldots, \mathrm{X} ; \lambda=1\right) / k_{\mathrm{B}} T}}{\int \mathrm{d} \mathbf{X} \mathrm{e}^{-U\left(\mathbf{r}_{1}, \mathbf{r}_{2}, \ldots, \mathrm{X} ; \lambda=0\right) / k_{\mathrm{B}} T}}$,

or equivalently, as a thermodynamic integration (Kirkwood, 1935),

$\mathcal{W}\left(\mathbf{r}_{1}, \mathbf{r}_{2}, \ldots\right)=\int_{0}^{1} \mathrm{~d} \lambda\left\langle\frac{\partial U}{\partial \lambda}\right\rangle_{(\lambda)}$,

where the subscript $\lambda$ on the bracket indicates that the average is taken with the potential energy $U(\lambda)$. It may be noted that the mass of the particles does not appear in Eqs. (15)-(16). As a consequence, the PMF is a thermodynamic quantity independent of time-scales. Because it governs all the underlying equilibrium properties in any effective dynamical model (GLE, LE, $\mathrm{BD})$, a rigorous formulation of equilibrium PMFs is a crucial first step in the formulation of a non-equilibrium transport theory. The following two sections will be entirely dedicated to this subject. Prior to this, we will briefly review the basis of mean-field theories of diffusion and how they relate to the important constraint of thermodynamic equilibrium.

\subsection{Mean-field theories}

Considering the dynamics of a many-body system from the perspective of a diffusion equation is attractive because, rather than having to extract the properties of the system from time averages taken over stochastic BD trajectories, one deals directly with probability densities and fluxes. However, the probability density of a many-body system, $P\left(\mathbf{r}_{1}, \mathbf{r}_{2}, \ldots ; t\right)$, evolves according the Smoluchowski Eq. (10). Such a multi-dimensional differential equation is virtually impossible to solve, even with today's computers. There is, however, a family of theoretical approaches that attempt to follow a similar route. To reduce the dimensionality of the many-body problem, these approaches consider only the non-equilibrium steady-state flux 
density of the particles

$\mathbf{J}(\mathbf{r})=-D\left[\nabla \rho(\mathbf{r})+\frac{\rho(\mathbf{r})}{k_{\mathrm{B}} T} \nabla \mathcal{W}^{\mathrm{eff}}(\mathbf{r})\right]$,

where $\rho(\mathbf{r})$ is the particle density, and $\mathcal{W}^{\text {eff }}(\mathbf{r})$ is some effective potential (Calef $\&$ Wolynes, 1983). This expression is similar to the flux describing the diffusion of a single particle diffusing in a potential $\mathcal{W}^{\text {eff }}(\mathbf{r})$. In effect, Eq. (17) turns the many-body problem into a pseudo-single-particle diffusion problem. This implies that the effective potential incorporates implicitly the influence of all the particles in the system. To obtain a closed set of equations, one must express the effective potential in terms of the average density,

$\mathcal{W}^{(\text {eff })}(\mathbf{r})=\mathbf{F}(\mathbf{r} ;[\rho(\mathbf{r}, t)])$

This means that $\mathcal{W}^{(\mathrm{eff})}(\mathbf{r})$ must be calculated using the number density $\rho(\mathbf{r}, t)$ as an input through the functional $\mathbf{F}$ (a function of a function). As the density depends on the effective potential, via Eq. (17), and the effective potential depends on the density, via Eq. (18), the two set of equations must be solved self-consistently. Different mean-field theories, varying in their level of sophistication, can be obtained by choosing different forms to approximate the effective potential in terms of the particle density $\rho(\mathrm{r})$. The approximation used in Eq. (18) to obtain a closed set of equations is usually referred to as a 'closure' [e.g. mean-spherical approximation (MSA), hypernetted chain (HNC), Percus-Yevick (PY)]. In application to ion channels, the most familiar example of such mean-field approaches is perhaps the Poisson-Nernst-Planck (PNP) theory (Kurnikova et al. 1999; Cardenas et al. 2000; Hollerbach et al. 2000, 2002; Schuss et al. 2001; Gillespie et al. 2002; Im \& Roux, 2002a; Kournanov et al. 2003; Mamonov et al. 2003), which originated from the electrodiffusion theory proposed by Onsager $(1926,1927)$.

Under equilibrium conditions (i.e. with no net flux), all the properties of any mean-field theory are directly determined by the choice of functional. There is a vast experience, going back to Debye \& Hückel (1923), Kirkwood (1935), Percus \& Yevick (1958), and Lebowitz \& Percus (1966) in the engineering of suitable functionals to represent the equilibrium structure of liquids. There have been numerous computational studies aimed at examining the ability of different functionals to accurately describe the equilibrium properties of a fluid, in the bulk phase (Fries \& Patey, 1985; Kusalik \& Patey, 1988), near a hard wall (Torrie et al. 1988; Berard \& Patey, 1992), near a complex molecular solute (Beglov \& Roux, 1996, 1997; Cortis et al. 1997; Kovalenko \& Hirata, 1998; Du et al. 1999), inside a pore (Beglov \& Roux, 1995). Excellent textbooks reviewing this subject are by McQuarrie (1976) and Hansen \& McDonald (1986). The ultimate assessment of the accuracy and usefulness of different closures is provided by comparison with so-called 'exact results' from computer simulations (Hansen \& McDonald, 1986). Years of experience with this subject have shown that several aspects of the structures of complex liquids can be reproduced with quantitative accuracy by all-atom MD (Hansen \& McDonald, 1986; Allen \& Tildesley, 1989) but are only represented qualitatively, even by the most sophisticated meanfield integral equation theories. There exists no exact closed-form functional expression for the effective potential of a many-body system and an intrinsic limitation of this family of approaches is that the underlying equilibrium properties have unavoidably an approximate mean-field character. The impact of this approximation on non-equilibrium properties is unknown. We will return to PNP electrodiffusion theory in Section 5.2.4 and its application to ion permeation through OmpF porin in Section 6.3.3. 


\section{Solvation free energy and electrostatics}

The previous section established that the PMF, which governs the effective dynamics of a reduced set of particles, is equivalent to a configuration-dependent solvation free energy. Therefore, one can expect that a key quantity in ion permeation will be some sort of multi-ion PMF, although Section 4 will further clarify this concept considerably. In liquid water, the importance of electrostatic interactions essentially dictates the magnitude of the solvation free energy of charged species. The electrostatic contribution to the solvation free energy of biomolecular solutes is often modeled on the basis of classical continuum electrostatics (Honig \& Nicholls, 1995). This approximation, in which the polar solvent is represented as a structureless continuum dielectric medium, was originally pioneered by Born (1920) to calculate the hydration free energy of spherical ions. Although the classical continuum approximation breaks down at length-scales of a few angstroms, it remains an exceedingly useful approach to formulate many factors concerned with long-range electrostatic effects that play a crucial role on ion permeation. In the following, we will use a continuum electrostatic approximation to establish some fundamental results concerning the importance of the low dielectric barrier presented by the lipid membrane and the transmembrane potential.

\section{I Microscopic view of the Born model}

According to the Born model (1920), the electrostatic contribution to the solvation free energy of an ion in liquid water is

$\Delta G_{\text {elec }}=\frac{1}{2} \frac{q^{2}}{R_{\text {ion }}}\left(\frac{1}{\varepsilon_{\mathrm{w}}}-1\right)$

where $q$ is the charge of the ion, $R_{\mathrm{ion}}$ its radius, and $\varepsilon_{\mathrm{w}}$ is the macroscopic dielectric constant of liquid water. A simple application of the Born model indicate that the electrostatic contribution to the solvation free energy of a small ion is on the order of $100 \mathrm{kcal} / \mathrm{mol}$, in qualitative agreement with experiments (Noyes, 1962). The success of the Born model demonstrates that the solvation free energy of an ion in water is largely dominated by electrostatic interactions arising from the polarization of the high dielectric solvent. The Born model was later extended by Kirkwood (1934) and Onsager (1936) for the treatment of arbitrary charge distributions inside a spherical cavity. Nowadays, the treatment of solutes of arbitrary shape is possible with the use of powerful computers and numerical methods (see Section 3.3 below).

To gain insight into the significance of the continuum electrostatic approximation, it is helpful to examine how the Born model arises at the microscopic level (Rashin \& Honig, 1985; Hirata et al. 1988; Jayaram et al. 1989; Roux et al. 1990). The electrostatic solvation free energy can be calculated as the reversible work needed to charge-up an ion in the solvent, according to Kirkwood's thermodynamics integration (Kirkwood, 1935),

$\Delta G_{\text {elec }}=\int_{0}^{q} \mathrm{~d} q^{\prime}\left\langle\frac{\partial U}{\partial q}\right\rangle_{\left(q^{\prime}\right)}$,

where $U$ is the potential energy of the system (comprising one ion surrounded by solvent molecules). The ion interacts with all the charges in the solvent molecules and the charging free energy can be written as

$\Delta G_{\text {elec }}=\int_{0}^{q} \mathrm{~d} q^{\prime} \int \mathrm{d} \mathbf{r} \frac{1}{r}\left\langle\rho_{\text {elec }}\left(r ; q^{\prime}\right)\right\rangle$, 


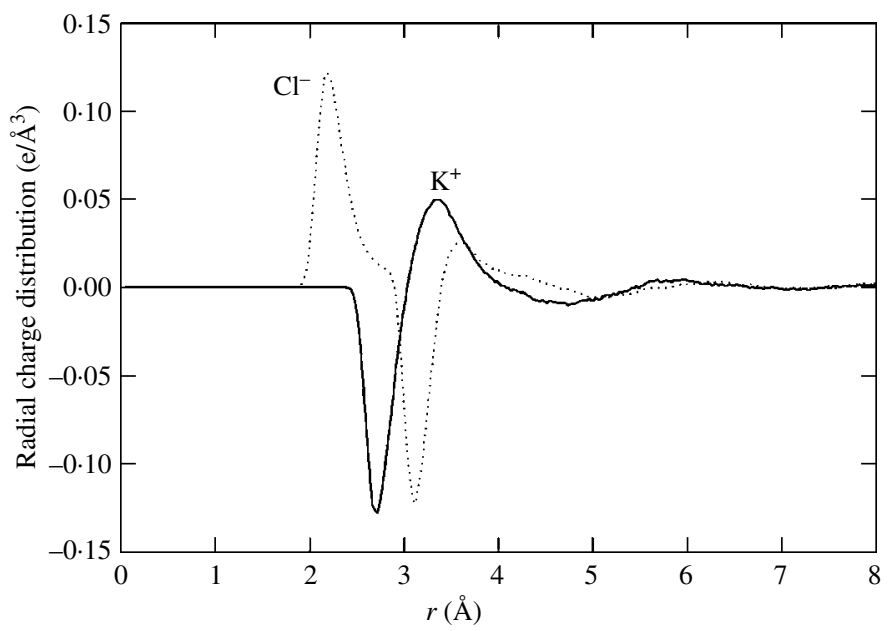

Fig. 4. Radial distribution of the solvent charge around a $\mathrm{K}^{+}$and a $\mathrm{Cl}^{-}$in bulk water. Around $\mathrm{K}^{+}$(solid line), the solvent charge distribution is dominated by the large negative peak at $r=2.6 \AA$ arising from the electronegative oxygen of the surrounding water molecules. Around $\mathrm{Cl}^{-}$(dotted line), the solvent charge distribution is dominated by the large positive at $r=2 \cdot 2 \AA$ peak arising from the electropositive hydrogens of the surrounding water molecules; the peak at $r=3.7 \mathrm{~A}$ arises from the second hydrogen in a water molecule. Details of the molecular dynamics simulations are given in Roux (1996).

where the quantity $\left\langle\rho_{\text {elec }}\left(r ; q^{\prime}\right)\right\rangle$ is the average charge distribution function of the solvent around the ion of charge $q^{\prime}$. Typically, the solvent charge distribution function is sharply peaked at some distance $r=R_{\mathrm{ion}}$, which corresponds to the radius of the ion, and nearly zero everywhere else. Examples of the solvent charge distributions around $\mathrm{K}^{+}$and $\mathrm{Cl}^{-}$calculated from $\mathrm{MD}$ simulations are shown in Fig. 4. A reasonable approximation to derive a closed form expression for $\Delta G_{\text {elec }}$ in Eq. (21) is to substitute the slowly varying integrand $1 / r$ by its value at the dominant peak of the solvent charge distribution $1 / R_{\mathrm{ion}}$, yielding,

$\Delta G_{\text {elec }} \approx \int_{0}^{q} \mathrm{~d} q^{\prime} \frac{1}{R_{\text {ion }}} \int \mathrm{d} \mathbf{r}\left\langle\rho_{\text {elec }}\left(r ; q^{\prime}\right)\right\rangle$.

The remaining integral over the solvent charge distribution function can be evaluated in closed form. The total electrostatic potential at large distance is $q^{\prime} / \varepsilon_{\mathrm{w}} r$. By virtue of Gauss's theorem, this implies that the total charge enclosed by a spherical surface of radius $r$ is $q^{\prime} / \varepsilon_{\mathrm{w}}$. This corresponds to the charge of the ion, $q^{\prime}$, plus the charge arising from the solvent polarization, $q^{\prime}\left(1 / \varepsilon_{\mathrm{w}}-1\right)$. It follows that the total charge from the solvent obeys exactly the sum rule,

$\int \mathrm{d} \mathbf{r}\left\langle\rho_{\text {elec }}\left(r ; q^{\prime}\right)\right\rangle=q^{\prime}\left(\frac{1}{\varepsilon_{\mathrm{w}}}-1\right)$.

The Born model of ion solvation given in Eq. (19) is obtained after substitution of this expression in Eq. (22) followed by an integration over the charge $q^{\prime}$ yielding $\frac{1}{2} q^{2}$. In reality, the solvent charge distribution function is distributed over a microscopic region of space of finite dimension (see Fig. 4). This shows that the optimal value for the Born radius $R_{\text {ion }}$, which corresponds to the peak in $\left\langle\rho_{\text {elec }}\left(r ; q^{\prime}\right)\right\rangle$, is not a property of the ion alone. Born radii are effective semi-phenomenological parameters depending on both the charge of the ion and the atomic structure of the solvent molecules (Roux et al. 1990). They are not equivalent to the Pauling ionic 
radii, which were deduced from analysis of salt crystals (Pauling, 1960), but empirical parameters that must be adjusted to yield the solvation free energy accurately. These parameters are typically not transferable from one environment to another.

\section{2 lon-ion interactions in bulk solution}

As discussed above, the Born model is able to capture the dominant contributions to the solvation free energy of a single ion in liquid water. What is, then, the character of the interactions between ions in bulk water and how do they relate to the simple laws of classical continuum electrostatics? From the perspective of statistical mechanics, the water-mediated ion-ion effective interactions should be represented by a PMF, which is related to the pair correlation function (Nienhuis \& Deutch, 1972; Chandler, 1977; Pettitt \& Rossky, 1986). At a large distance $r$, the ion-ion PMF has the following asymptotic form (Chandler, 1977; Pettitt \& Rossky, 1986)

$\lim _{r \rightarrow \infty} \mathcal{W}_{\text {bulk }}^{(i j)}(r) \rightarrow \frac{q_{i} q_{j}}{\varepsilon_{\mathrm{w}} r}$,

where $q_{i}$ and $q_{j}$ are the charge of the ions, and $\varepsilon_{\mathrm{w}}$ is the dielectric constant of bulk water. Therefore, at large distances the ion-ion PMF does behave as a simple coulomb interaction shielded by the dielectric constant of water, in accord with classical electrostatics (Jackson, 1962). However, the PMF between two small ions is not represented accurately by this form at all distances. At small distances, there are important structural packing effects caused by the granularity and the finite size of the water molecules (Pettitt \& Rossky, 1986; Guàrdia \& Pardró, 1991; Llano-Restrepo \& Chapman, 1994). For example, the effective interactions between $\mathrm{K}^{+}$ and $\mathrm{Cl}^{-}$in liquid water shown in Fig. 5 displays damped oscillations of considerable complexity. It is customary to express the ion-ion PMF as (Pettitt \& Rossky, 1986)

$\mathcal{W}_{\text {bulk }}^{(i j)}(r)=w_{\text {st }}^{(i j)}(r)+\frac{q_{i} q_{j}}{\varepsilon_{\mathrm{w}} r}$,

where $w_{\mathrm{sr}}(r)$ represents some short-range solvent-mediated ion-ion interaction. Such potentials may be useful approximations to simulate ionic solution with many ions (Im et al. 2000; Moy et al. 2000),

$\mathcal{W}_{\text {bulk }}^{(i j)}\left(\mathbf{r}_{1}, \mathbf{r}_{2}, \ldots\right)=\sum_{i<j} w_{\mathrm{sr}}^{(i j)}\left(\left|\mathbf{r}_{i}-\mathbf{r}_{j}\right|\right)+\frac{q_{i} q_{j}}{\varepsilon_{\mathrm{w}}\left|\mathbf{r}_{i}-\mathbf{r}_{j}\right|}$.

If a simple Lennard-Jones 6-12 potential is taken to represent the short-range interaction potential, then the model is equivalent to the restricted primitive model with soft core, which has been extensively used in numerous statistical mechanical studies of ionic solutions (Ramanathan \& Friedman, 1971; Ermak, 1975; Wood \& Friedman, 1987; Jardat et al. 1999).

\subsection{Continuum electrostatics and the PB equation}

We have reviewed some basic aspects of ion solvation and solvent-mediated ion-ion interactions in bulk solution and highlighted the fact that the solvation free energy of polar systems in water is often dominated by electrostatics. The treatment of complex molecular systems of arbitrary geometries with classical continuum electrostatic methods follows essentially the same principles. 

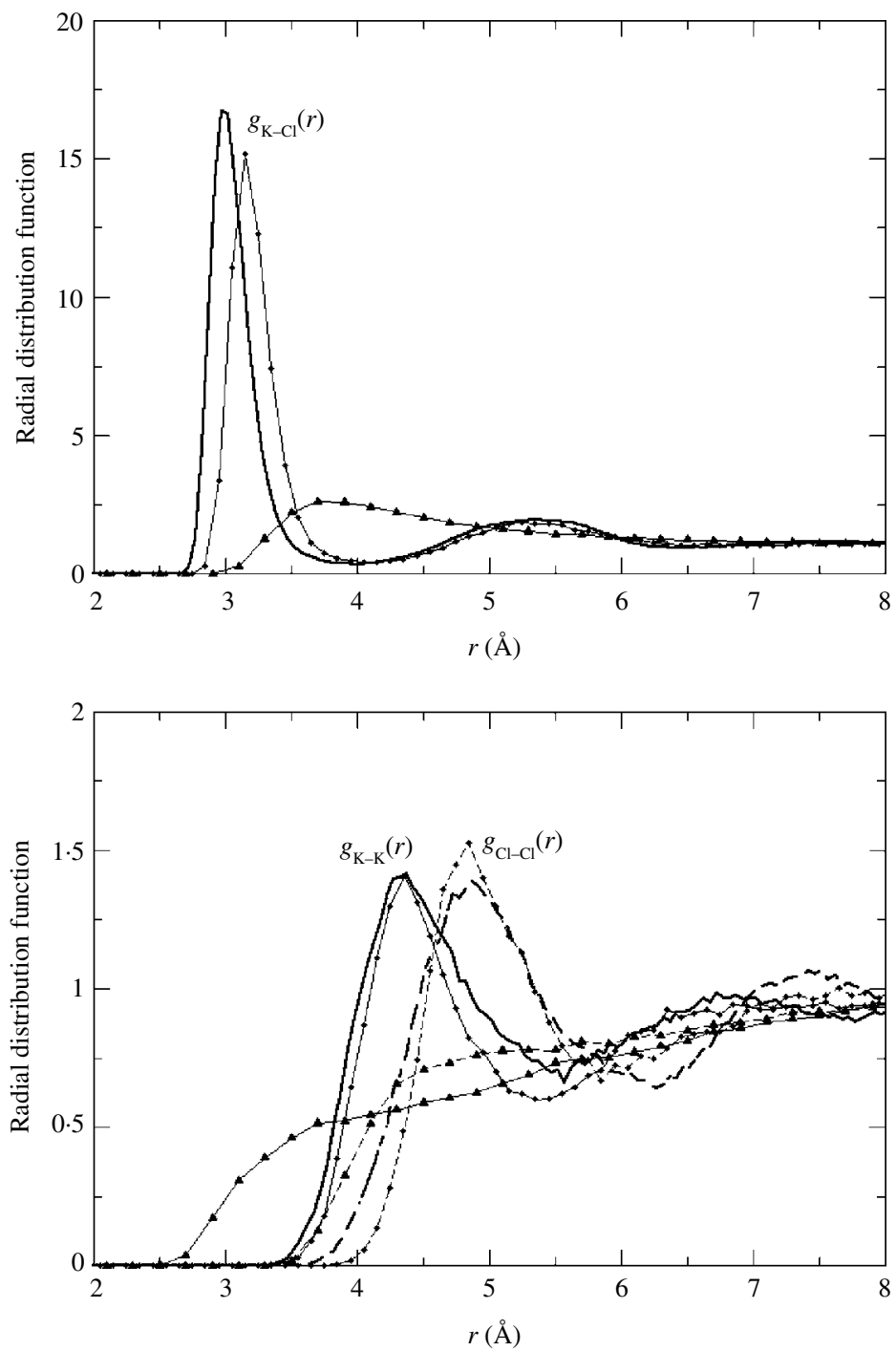

Fig. 5. Ion-ion radial distribution functions from molecular dynamics (thick lines), Brownian dynamics (BD) simulation with short-range ion-ion interaction $w_{\text {sr }}$ (with circles), BD with the primitive model (with triangles).

It is made possible by mapping the problem onto a discrete grid and using finite-difference relaxation numerical algorithms to solve the PB equation (Warwicker \& Watson, 1982a; Klapper et al. 1986). To clarify the microscopic basis of the PB equation, let us consider a molecular solute immersed in an aqueous salt solution. Let us collectively designate the coordinates $\left\{\mathbf{r}_{1}, \ldots, \mathbf{r}_{p}, \ldots\right\}$ of the fixed solute as $\mathbf{X}_{\mathrm{p}}$, where each atom $p$, located at $\mathbf{r}_{p}$ carries a partial charge $q_{p}$. It is assumed that the average spatial density of the mobile ions in the aqueous regions around the microscopic solute depends only on the local electrostatic potential $\phi(\mathbf{r})$

$\left\langle\rho_{\alpha}(\mathbf{r})\right\rangle=\bar{\rho}_{\alpha} \mathrm{e}^{-q_{\alpha} \phi(\mathbf{r}) / k_{\mathrm{B}} T}$, 
where $\alpha$ refers to a specific type of mobile ion, and $\bar{\rho}_{\alpha}$ is its number density in the bulk solution. Such an approximation, which was pioneered by Gouy (1910), Chapman (1913), and Debye \& Hückel (1923), is expected to be valid at moderately low ionic concentration when core-core interactions between the mobile ions may be neglected. The total space-dependent charge density from the mobile ions is

$$
\left\langle\rho_{\text {ions }}(\mathbf{r})\right\rangle=\mathrm{e}^{-U_{\text {core }}(\mathbf{r}) / k_{\mathrm{B}} T} \sum_{\alpha} q_{\alpha} \bar{\rho}_{\alpha} \mathrm{e}^{-q_{\alpha} \phi(\mathbf{r}) / k_{\mathrm{B}} T},
$$

where $U_{\text {core }}(\mathbf{r})$ is a core-repulsive potential excluding the ions from the interior of the solute. The nonlinear form of the $\mathrm{PB}$ equation is obtained when $\left\langle\rho_{\text {ions }}(\mathbf{r})\right\rangle$ is inserted explicitly in the Poisson equation for macroscopic media (Jackson, 1962),

$\boldsymbol{\nabla} \cdot[\varepsilon(\mathbf{r}) \nabla \phi(\mathbf{r})]=-4 \pi\left(\rho_{\mathrm{p}}(\mathbf{r})+\sum_{\alpha} q_{\alpha} \bar{\rho}_{\alpha} \mathrm{e}^{-U_{\text {core }}(\mathrm{r}) / k_{\mathrm{B}} T} \mathrm{e}^{-q_{\alpha} \phi(\mathbf{r}) / k_{\mathrm{B}} T}\right)$,

where $\varepsilon(\mathbf{r})$ is the position-dependent dielectric constant at point $\mathbf{r}$, and $\rho_{\mathrm{p}}(\mathbf{r})$ is the charge density of the solute

$\rho_{\mathrm{p}}(\mathbf{r})=\sum_{p} q_{p} \delta\left(\mathbf{r}-\mathbf{r}_{p}\right)$.

Linearization of Eq. (29) with respect to the potential $\phi$ yields the familiar Debye-Hückel approximation or linearized PB equation (Debye \& Hückel, 1923; Fowler \& Guggenheim, 1939)

$\boldsymbol{\nabla} \cdot[\varepsilon(\mathbf{r}) \nabla \phi(\mathbf{r})]-\bar{\kappa}^{2}(\mathbf{r}) \phi(\mathbf{r})=-4 \pi \rho_{\mathrm{p}}(\mathbf{r})$,

where $\bar{\kappa}^{2}(\mathbf{r})$ is the space-dependent screening factor which varies from zero, in the solventexcluded regions, to $4 \pi \sum_{\alpha} q_{\alpha}^{2} \bar{\rho}_{\alpha} / k_{\mathrm{B}} T$, in the bulk solvent. The electrostatic contribution to the solvation free energy is calculated from

$\Delta G_{\text {elec }}\left(\mathbf{X}_{\mathrm{p}}\right)=\frac{1}{2} \sum_{p} q_{p} \phi_{\mathrm{rf}}\left(\mathbf{r}_{p}\right)$

where

$\phi_{\text {rf }} \equiv \phi_{\text {syst }}-\phi_{\text {vac }}$

is the reaction field; $\phi_{\text {syst }}$ is the electrostatic potential in the full system, calculated from PB Eq. (29) with the complex dielectric boundaries defined from the non-uniform dielectric constant $\varepsilon(\mathbf{r})$, and $\phi_{\text {vac }}$ is the electrostatic potential calculated in vacuum with a uniform dielectric constant of 1 .

Several programs are available for computing the electrostatic potential using this approach, e.g. DelPhi (Klapper et al. 1986; Gilson et al. 1987), UHBD (Davis et al. 1991), and the PBEQ module (Nina et al. 1997; Im et al. 1998) incorporated in the simulation program CHARMM (Brooks et al. 1983). As shown by comparisons to simulations with explicit water molecules, classical continuum electrostatic approximations are remarkably successful in reproducing the electrostatic contribution to the solvation free energy of small solutes (Jean-Charles et al. 1991; Sitkoff et al. 1994), amino acids (Nina et al. 1997), and nucleic acids (Banavali \& Roux, 2002). 
Furthermore, the PB equation (linear and nonlinear) is a particularly simple and powerful approach to address questions about the influence of salt on complex biological systems (Sharp \& Honig, 1995; Misra \& Honig, 1996; Misra et al. 1998). Excellent reviews of classical electrostatics are, for example, Partenskii \& Jordan (1992b), Honig \& Nicholls (1995), and Warshel \& Papazyan (1998).

The total solvation free energy of a molecular system may be expressed as the reversible work for its complete materialization into the bulk solvent in a step-by-step process (Roux \& Simonson, 1999). In particular, one can envision the solvation free energy of a solute as the total reversible work needed to first create a neutral van der Waals cavity into the solvent, and subsequently switch-on the electrostatic interactions between the solute and the solvent molecules,

$\Delta G=\Delta G_{\mathrm{np}}+\Delta G_{\mathrm{elec}}$.

While the electrostatic contribution is calculated via Eq. (32) as described above, the non-polar contribution is generally approximated in terms of the solvent-accessible surface area (SASA), $\mathcal{S}$,

$\Delta G_{\mathrm{np}}=\gamma_{\mathrm{s}} \mathcal{S}$

where $\gamma_{\mathrm{s}}$ is the surface tension of the solvent. Both contributions depend on the configuration of the solute.

In the case of simple monatomic ions, the total solvation free energy, $\Delta G$, is equivalent to the intrinsic excess chemical potential $\Delta \bar{\mu}$. Including all contributions, the solvation free energy of an ion in a salt solution may be approximated as

$\Delta \bar{\mu}=\gamma_{\mathrm{s}} 4 \pi R_{\mathrm{ion}}^{2}+\frac{q^{2}}{2 R_{\mathrm{ion}}}\left(\frac{1}{\varepsilon_{\mathrm{w}}}-1\right)-\frac{q^{2} \kappa}{2 \varepsilon_{\mathrm{w}}\left(1+\kappa R_{\mathrm{ion}}\right)}$

(for the derivation see McQuarrie, 1976). For the sake of simplicity, a unique radius $R_{\text {ion }}$ was assumed in Eq. (36). However, the parameterization of an accurate continuum electrostatic model requires the development of optimal sets of radii to construct the cavity as well as the space-dependent functions $\bar{\kappa}^{2}(\mathbf{r})$ and $\varepsilon(\mathbf{r})$. As illustrated in the microscopic derivation of the Born model, the dielectric boundary is closely related to the nearest density peak in the solute-solvent distribution function (Roux et al. 1990). Different schemes for obtaining atomic radii optimizing to reproduce the solvation free energy of a collection of molecules have been suggested (Sitkoff et al. 1994; Nina et al. 1997). The spatial dependence of $\bar{\kappa}^{2}(\mathbf{r})$ is often assumed to be similar to that of $\varepsilon(\mathbf{r})$, though the region accessible to the mobile ions does not have to coincide precisely with the region accessible to high dielectric water. The atomic radii that are optimal for setting the protein-water dielectric interface may differ from the optimal atomic radii needed to set the ion-accessible region near the protein surface (Im \& Roux, 2002b).

\subsection{Limitations of continuum dielectric models}

Before going further, it is useful to expand a little more on the limitations of continuum dielectric models leading to Eq. (31). One important aspect of linear macroscopic continuum electrostatics is that the average polarization density $\mathbf{P}(\mathbf{r})$, at point $\mathbf{r}$, obeys a linear relation involving the local electrostatic field, $\mathbf{E}(\mathbf{r})$

$\mathbf{P}(\mathbf{r})=\left(\frac{\varepsilon(\mathbf{r})-1}{4 \pi}\right) \mathbf{E}(\mathbf{r})$ 
where $\mathbf{E}=-\boldsymbol{\nabla} \phi$ is the total electrostatic field. As a consequence, $\mathbf{P}(\mathbf{r})$ and $\mathbf{E}(\mathbf{r})$ are co-linear vectors. In typical application of the $\mathrm{PB}$ equation, the space-dependent dielectic $\varepsilon(\mathbf{r})$ is assigned a value of 80 in bulk water, of 2 in the lipid hydrocarbon, and a value between 2 and 10 for the protein (Honig \& Nicholls, 1995). It should be emphasized that a local constitutive relation, such as Eq. (37) is an approximation. A prerequisite to derive Eq. (37) from microscopic considerations is that the total field, $\mathbf{E}$, must be weak and vary only on a length-scale that is much longer than the correlation length of the liquid (Nienhuis \& Deutch, 1971; Chandler, 1977; Partenskii \& Jordan, 1992b; Beglov \& Roux, 1996). Generalizations of Eq. (37) often involve a spatial convolution with a non-local dielectric response function, $\varepsilon\left(\mathbf{r}, \mathbf{r}^{\prime}\right)$. Such non-locality should not be surprising. After all, the dielectric constant is a macroscopic concept, born out of intrinsically non-local electrostatic interactions. Generally, the dielectric response function is sharply peaked at $\mathbf{r}=\mathbf{r}^{\prime}$, and decays rapidly to zero when $\left|\mathbf{r}-\mathbf{r}^{\prime}\right|$ becomes larger than the microscopic correlation length of the liquid (typically a few $\AA$ for liquid water). Assuming that the dielectric response function is local is an approximation that is valid only to describe electrostatic features that are established over lengthscales that are larger than the correlation length of the liquid. For example, the electrostatic potential arising from the average solvent polarization in the neighborhood of a molecular solute may exhibit strong oscillations that are considerably more complex than those expected from a continuum approximation (Beglov \& Roux, 1996). Furthermore, additional phenomena related to non-linear dielectric saturation of the solvent can also take place when the local fields are too large (Warshel \& Åqvist, 1991; Beglov \& Roux, 1996). The concept of a local dielectric constant to represent the electrostatic response of a fluctuating macromolecule encounters fundamental difficulties, which further highlight the limitations of continuum approximations (Gilson \& Honig, 1986; King et al. 1991; Warshel \& Papazyan, 1998; Schutz \& Warshel, 2001; Simonson, 2003). While continuum electrostatics is a useful and valid approximation to describe broad features related at moderate and large lengthscales, it is worth keeping these considerations in mind when such an approximation is applied to microscopic systems.

\subsection{The dielectric barrier}

Cell membranes are supramolecular sheet-like structures formed by lipid molecules possessing long non-polar hydrocarbon chains attached to a polar moiety (Gennis, 1989). The hydrocarbon chains of the lipid molecules form a 20-30 $\AA$ non-polar (low dielectric) core at the center of the bilayer. A pioneering study by Parsegian (1969) showed that the presence of the low dielectric membrane has a great impact on ion permeation. He calculated the free energy of transfer of an ion to the center of a planar membrane using a continuum electrostatic approximation. In his model, the non-polar core formed by the hydrocarbon chains is approximated by a slab of featureless material having a dielectric constant of 2 while the aqueous solutions are represented by regions having a dielectric of 80 . The main conclusion was that there is a large energy barrier opposing the passage of an ion across the membrane. To illustrate this important concept, we calculated the free-energy profile of a $\mathrm{K}^{+}$across a low dielectric membrane. The result is shown in Fig. 6a. It is observed that the free energy to transfer an ion to the center of the membrane is $\sim 38 \mathrm{kcal} / \mathrm{mol}$. This large free-energy barrier, opposing the passage of an ion across the low dielectric membrane, is widely referred to as the 'dielectric barrier' (Parsegian, 1969). The dielectric barrier is a fundamental impediment to ion permeation across cell membranes. Ion channels are specialized proteins whose primary role is to help overcome the dielectric barrier 

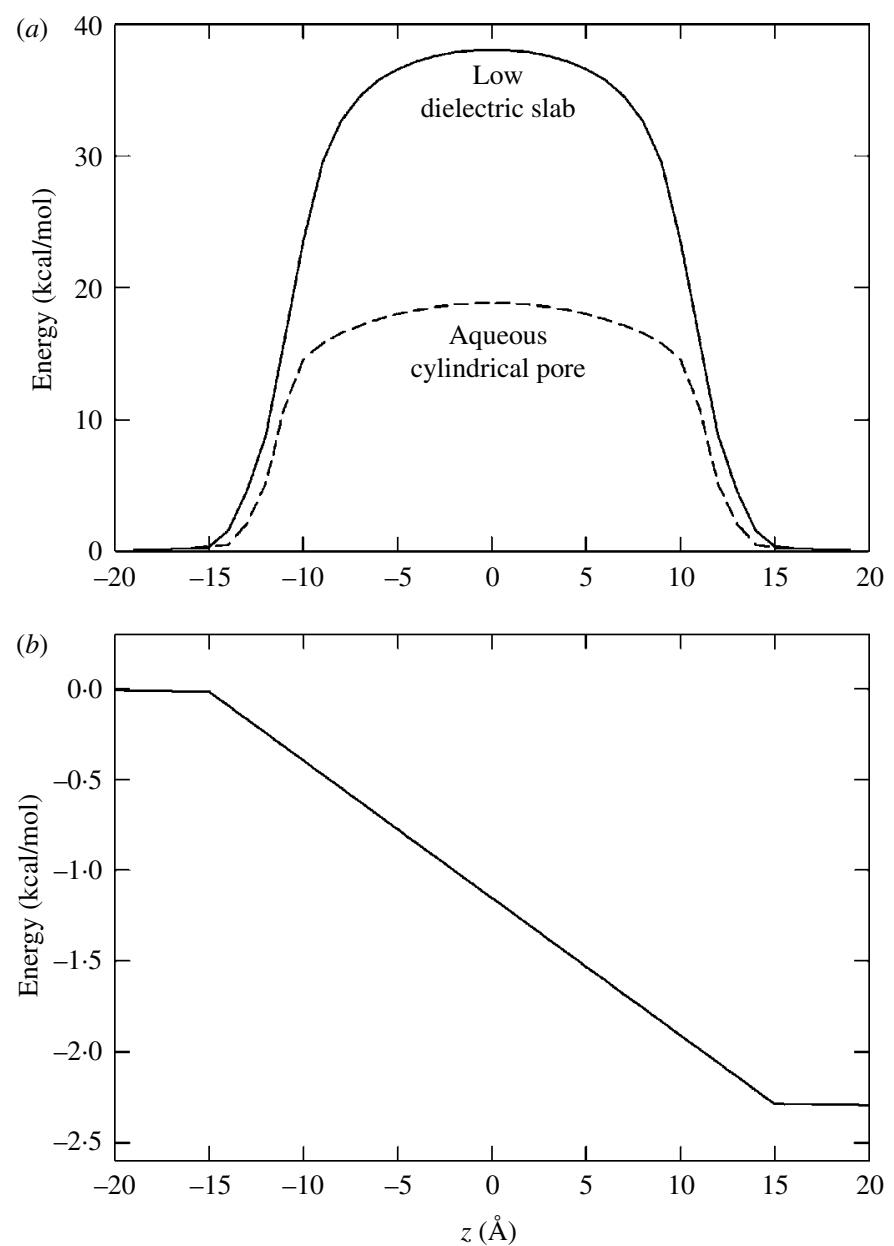

Fig. 6. Illustration of the important concepts in membrane electrostatics. (a) The dielectric barrier. The solid line is the electrostatic reaction-field free energy of a $\mathrm{K}^{+}$ion going across a $30 \AA$ membrane represented by a structureless continuum medium of dielectric 2 . The dashed line is the electrostatic reaction-field free energy of a $\mathrm{K}^{+}$ion going through a $3 \AA$ diameter cylindrical aqueous pore of dielectric constant 80 . The finite-difference calculations were done using the PBEQ module (Nina et al. 1997; Roux, 1997; Im et al. 1998) of the biomolecular simulation program CHARMM (Brooks et al. 1983). The total electrostatic potential was calculated at each point of the grid by solving the finite difference Poisson equation. No electrolyte was included in the bulk solution. (b) The transmembrane potential in the linear field approximation with a potential difference of $100 \mathrm{mV}$.

presented by the cell membrane. Using a continuum electrostatic model, Levitt (1978) and Jordan (1981) showed that even the presence of small pore-containing high-dielectric medium can help to reduce the dielectric barrier significantly. This important observation is illustrated in Fig. $6 a$ for cylindrical pore of $3 \AA$ radius. Although such a model cannot account for ionic selectivity, it highlights the importance of (high dielectric) water molecules along the permeation pathway. Since this early work, similar electrostatic calculations based on channel models with more complex shapes have been carried out (Jordan, 1984; Jordan \& Vayl, 1985). However, it is not possible to go beyond this without further structural information about ion channels at the atomic level. 


\subsection{The transmembrane potential and the PB-V equation}

The transmembrane potential is an important driving force governing the movement of charged species across the cell membrane. As first discussed by Nernst (1889), a membrane potential appears spontaneously between two ionic solutions separated by a semi-permeable membrane as the result of a balance between the entropic tendency to homogenize the system and the necessity to maintain local charge neutrality. The struggle between these two opposing forces takes place near the semi-permeable membrane, and the potential difference is the result of this interfacial phenomena. Alternatively, it is possible to impose a potential difference across an impermeable membrane in the laboratory using an external electromotive force and ionexchanging electrodes. Both situations correspond to molecular systems in thermodynamic equilibrium.

At the microscopic level the transmembrane potential arises from a small charge imbalance distributed in the neighborhood of the membrane-solution interface. The net charge per area for a transmembrane potential of $100 \mathrm{mV}$ corresponds roughly to only one atomic unit charge per surface of $130 \times 130 \AA^{2}$. Since a physiological salt concentration of $150 \mathrm{~mm}$ corresponds to approximately one cation-anion pair per cubic volume of $22 \times 22 \times 22 \AA^{3}$, the membrane potential arises from a strikingly small accumulation of net charge compared to the bulk ion density. As illustrated in Fig. 6b, the transmembrane potential has a particularly simple linear form in the case of a perfectly planar membrane. Because there are no charges in the low dielectric membrane, the potential is traditionally assumed to vary linearly across the membrane (constant field approximation). Therefore, the potential is constant on both sides of the membrane with a potential difference of $V_{\mathrm{mp}}$. A constant field such as shown in Fig. $6 b$ is, however, probably inaccurate in the case of wide aqueous pores of irregular shapes. It is thus necessary to develop a more general treatment of the membrane potential.

Let us consider a protein embedded in an impermeable membrane separating two salt solutions. The system is illustrated in Fig. 7. Let $\mathbf{X}_{\mathrm{p}}$ represent the configuration of the protein (a few ions and water molecules at fixed positions could also be included explicitly as part of the microscopic system). An external electromotive force (EMF), connected to sides I and II by two electrodes, gives rise to the membrane potential $V_{\mathrm{mp}}$. For simplicity, it is assumed that the electrodes are ideal and that one ionic species can be transported from one side to the other through the external electrical circuit. In a real system, the ionic species is not transported per se through the external circuit by the EMF. The electrical communication between the external circuit and the electrolyte is through electrode reactions of the type: $\mathrm{AgCl}+\mathrm{e}^{-} \leftrightharpoons \mathrm{Ag}+\mathrm{Cl}^{-}$, i.e. the chloride anion is chemiabsorbed at the surface of the electrode on one side, yielding one electron which is transported through the electric circuit, leading to the release of a chloride anion on the other side (Bockris \& Reddy, 1970). However, the exact detail of the electrode reaction is unimportant in the present development.

Assuming that the system is in thermodynamic equilibrium (there is no flux), the probability of any configuration is given according to the Boltzmann factor, $\exp \left[-U_{\text {tot }} / k_{\mathrm{B}} T\right]$, where the total potential energy in the system is $U_{\text {tot }}=U-Q_{\text {ext }} V_{\mathrm{mp}}$, where $U$ is the molecular potential energy (protein, membrane and salt solutions), and $-Q_{\text {ext }} V_{\mathrm{mp}}$ is the energy of the total net charge that has transited through the external EMF from side I to side II (there is a minus sign because the potential energy of a positive charge is decreasing as it travels from side I to side II through the EMF). Because any macroscopic charge imbalance in the bulk region would yield a prohibitively large energy, the net charge $Q_{\text {ext }}$ is very small and the salt solutions remain globally 


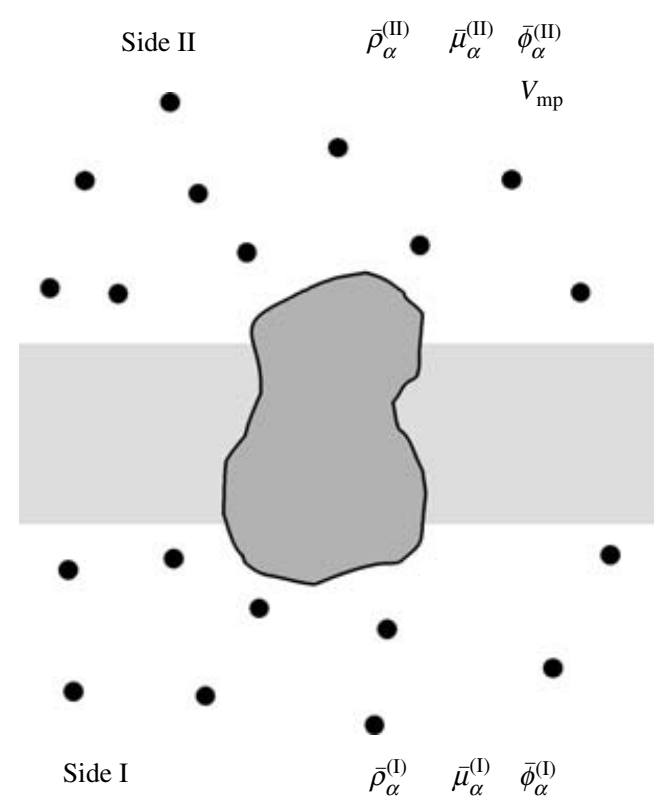

Fig. 7. Schematic representation of an intrinsic protein embedded in membrane under the influence of a transmembrane potential. The system is assumed to be in thermodynamic equilibrium. The transmembrane potential is 0 on side I and $V_{\mathrm{mp}}$ on side II. The density of the permeable ions, the excess chemical potential, and the average electrostatic potential, are respectively, $\bar{\rho}^{(\mathrm{S})}, \bar{\mu}^{(\mathrm{S})}$, and $\bar{\phi}^{(\mathrm{S})}$ on side $\mathrm{S}$ (I or II) of the membrane.

neutral. The bulk density of ions of type $\alpha$ is $\bar{\rho}_{\alpha}^{\mathrm{I}}$ and $\bar{\rho}_{\alpha}^{\mathrm{II}}$ on sides I and II of the membrane respectively. The equilibrium condition for the ionic species $\alpha$ transported by the EMF is

$\frac{\bar{\rho}_{\alpha}^{(\mathrm{I})}}{\overline{\boldsymbol{\rho}}_{a}^{(\mathrm{II})}}=\frac{\mathrm{e}^{-\bar{\mu}_{\alpha}^{(\mathrm{I})} / k_{\mathrm{B}} T}}{\mathrm{e}^{-\left(\bar{\mu}_{\alpha}^{(\mathrm{II})}-q_{\alpha} V_{\mathrm{mp}}\right) / k_{\mathrm{B}} T}}$,

where $\bar{\mu}_{\alpha}^{(\mathrm{S})}$ is the excess chemical potential on side $\mathrm{S}$. The excess chemical potential $\bar{\mu}_{\alpha}^{(\mathrm{S})}$ is composed of two contributions, the intrinsic excess chemical potential, $\Delta \bar{\mu}_{\alpha}^{(\mathrm{S})}$, arising from local interactions of the ion with the surrounding particles in the bulk (see above), and the interaction with the overall electrostatic potential in the bulk solution (far away from the membrane), $\bar{\phi}^{(\mathrm{S})}$,

$\bar{\mu}_{\alpha}^{(\mathrm{S})}=\Delta \bar{\mu}_{\alpha}^{(\mathrm{S})}+q_{\alpha} \bar{\phi}^{(\mathrm{S})}$

The electrostatic potential $\bar{\phi}^{(S)}$ results from the very small net charge imbalance carried by the EMF. In an electrolyte solution, this net charge is distributed at the interfacial boundaries. Equations (38) and (39) imply that the difference in the average electrostatic potential between sides I and II is

$\bar{\phi}^{(\mathrm{II})}-\bar{\phi}^{(\mathrm{I})}=V_{\mathrm{mp}}+\frac{k_{\mathrm{B}} T}{q_{\alpha}} \ln \left[\frac{\bar{\rho}_{\alpha}^{(\mathrm{I})}}{\bar{\rho}_{\alpha}^{(\mathrm{II})}}\right]+\frac{1}{q_{\alpha}}\left[\Delta \bar{\mu}_{\alpha}^{(\mathrm{I})}-\Delta \bar{\mu}_{\alpha}^{(\mathrm{II})}\right]$.

The intrinsic excess chemical potential $\Delta \bar{\mu}_{\alpha}^{(\mathrm{S})}$ depends on the ionic strength of the solution (see Section 3.3). Experimentally, one tries to control the composition of the solutions so that 
$\bar{\phi}^{(\mathrm{II})}-\bar{\phi}^{(\mathrm{I})} \approx V_{\mathrm{mp}}$. In the following, we will assume that those conditions are fulfilled and that the difference in electrostatic potential simply corresponds to the EMF.

We now focus our attention on the total electrostatic contribution to the free energy $G_{\text {elec }}$. This contribution may be calculated as the step-by-step reversible thermodynamic work required to first impose the membrane potential $V_{\mathrm{mp}}$, and then switch on the electrostatic interactions between the protein and its surrounding. Introducing the coupling parameter $\lambda$, varying between 0 and 1 to scale the protein charges, the electrostatic free energy may be expressed conveniently as

$$
\begin{aligned}
\Delta G_{\text {elec }}\left(\mathbf{X}_{\mathrm{p}}\right) & =\int_{0}^{V_{\mathrm{mp}}} \mathrm{d} V_{\mathrm{mp}}\left\langle\frac{\partial U_{\mathrm{tot}}}{\partial V_{\mathrm{mp}}}\right\rangle_{\left(V_{\mathrm{mp}}, \lambda=0\right)}+\int_{0}^{1} \mathrm{~d} \lambda\left\langle\frac{\partial U_{\mathrm{tot}}}{\partial \lambda}\right\rangle_{\left(V_{\mathrm{mp}}, \lambda\right)} \\
& =-\int_{0}^{V_{\mathrm{mp}}} \mathrm{d} V_{\mathrm{mp}}\left\langle Q_{\text {ext }}\right\rangle_{\left(V_{\mathrm{mp}}, \lambda=0\right)}+\int_{0}^{1} \mathrm{~d} \lambda \sum_{p} q_{p} \phi\left(\mathbf{r}_{p} ; V_{\mathrm{mp}}, \lambda\right),
\end{aligned}
$$

where $p$ indicates the protein charges, and $\phi\left(\mathbf{r} ; V_{\mathrm{mp}}, \lambda\right)$ is the total average electrostatic potential at point $\mathbf{r}$, with protein charges scaled by $\lambda$, and imposed membrane potential $V_{\mathrm{mp}}$. To calculate $\phi\left(\mathbf{r} ; V_{\mathrm{mp}}, \lambda\right)$, we use the Poisson equation for macroscopic media (Jackson, 1962)

$\boldsymbol{\nabla} \cdot[\varepsilon(\mathbf{r}) \nabla \phi(\mathbf{r})]=-4 \pi \lambda \rho_{p}(\mathbf{r})-4 \pi\left\langle\rho_{\text {ions }}(\mathbf{r})\right\rangle_{\left(V_{\mathrm{mp}}, \lambda\right)}$,

where $\varepsilon(\mathbf{r})$ is a position-dependent dielectric constant equal to $\varepsilon_{\mathrm{w}}, \varepsilon_{\mathrm{w}}$, and $\varepsilon_{\mathrm{p}}$ in the bulk water, membrane, and protein regions, respectively. The dielectric boundary of the protein corresponds to the molecular surface which may be determined using effective atomic radii (Nina et al. 1997), and $\lambda \rho_{\mathrm{p}}(\mathbf{r})$ and $\left\langle\rho_{\text {ions }}(\mathbf{r})\right\rangle_{\left(V_{\mathrm{mp}}, \lambda\right)}$ are the charge density associated with the protein and mobile counter ions respectively. As indicated by the subscript $\left(V_{\mathrm{mp}}, \lambda\right)$, the density of the mobile ions in the salt solution on both sides of the membrane varies as a function of the membrane potential $V_{\mathrm{mp}}$ and the coupling parameter $\lambda$ (in the following, the subscript on the density of mobile ions will be omitted to simplify the notation). To make further progress it is necessary to have a microscopic description of the ion-charge densities in the bulk solutions. The number density of ions of type $\alpha$ at $\mathbf{r}$ on side $\mathrm{S}$ is

$\left\langle\rho_{\alpha}(\mathbf{r})\right\rangle=\bar{\rho}_{\alpha}^{(\mathrm{S})} \mathrm{e}^{-\left(\mu_{\alpha}(\mathbf{r})-\bar{\mu}_{\alpha}^{(S)}\right) / k_{\mathrm{B}} T}$,

where $\mu_{\alpha}(\mathbf{r})$ is the excess chemical potential of ion of type $\alpha$ at point $\mathbf{r}$ (this is equivalent to the reversible work to insert an ion at point $\mathbf{r}$ ). Neglecting ion-ion short-range interactions, the chemical potential of an ion of type $\alpha$ at point $\mathbf{r}$ is

$\mu_{\alpha}^{(\mathrm{S})}(\mathbf{r})=U_{\text {core }}(\mathbf{r})+\Delta \bar{\mu}_{\alpha}^{(\mathrm{S})}+q_{\alpha} \phi(\mathbf{r})$

where $\phi(\mathbf{r})$ is the total average electrostatic potential at point $\mathbf{r}$, and $U_{\text {core }}(\mathbf{r})$ is the repulsive core overlap potential excluding the ions from the interior of the protein or the membrane. The total charge density of the mobile ions at any point $\mathbf{r}$ is

$\left\langle\rho_{\text {ions }}(\mathbf{r})\right\rangle= \begin{cases}\mathrm{e}^{-U_{\text {core }}(\mathbf{r}) / k_{\mathrm{B}} T} \sum_{\alpha} q_{\alpha} \bar{\rho}_{\alpha}^{(\mathrm{I})} \mathrm{e}^{-q_{\alpha}\left(\phi(\mathbf{r})-\bar{\phi}^{(\mathrm{I})}\right) / k_{\mathrm{B}} T} & \text { if } \mathbf{r} \text { is on side I } \\ \mathrm{e}^{-U_{\text {core }}(\mathbf{r}) / k_{\mathrm{B}} T} \sum_{\alpha} q_{\alpha} \bar{\rho}_{\alpha}^{(\mathrm{II})} \mathrm{e}^{-q_{\alpha}\left(\phi(\mathbf{r})-\bar{\phi}^{(\mathrm{II})}\right) / k_{\mathrm{B}} T} & \text { if } \mathbf{r} \text { is on side II. }\end{cases}$

This expression is a generalization of Eq. (28). For physiological systems (i.e. a membrane potential on the order of $100 \mathrm{mV}$ and a salt concentration of $150 \mathrm{~mm}$ ), the argument of the 
exponentials in Eq. (45) is expected to be small and the exponential functions may be linearized (i.e. $\exp (x) \approx 1+x$ ). Assuming that $\bar{\phi}^{(\mathrm{I})}=0$ and $\bar{\phi}^{(\mathrm{II})}=V_{\mathrm{mp}}$, one has after linearization

$\left\langle\rho_{\text {ions }}(\mathbf{r})\right\rangle=\left(\frac{-\bar{\kappa}^{2}(\mathbf{r})}{4 \pi}\right)\left[\phi(\mathbf{r})-V_{\mathrm{mp}} \Theta(\mathbf{r})\right]$,

where $\Theta(\mathbf{r})$ is a Heaviside step-function equal to 0 on side I and equal to 1 on side II, and

$\bar{\kappa}^{2}(\mathbf{r})= \begin{cases}\left(4 \pi / k_{\mathrm{B}} T\right) \mathrm{e}^{-U_{\text {core }}(\mathbf{r}) / k_{\mathrm{B}} T} \sum_{\alpha} q_{\alpha}^{2} \bar{\rho}_{\alpha}^{(\mathrm{I})} & \text { if } \mathbf{r} \text { is on side I } \\ \left(4 \pi / k_{\mathrm{B}} T\right) \mathrm{e}^{-U_{\text {core }}(\mathbf{r}) / k_{\mathrm{B}} T} \sum_{\alpha} q_{\alpha}^{2} \bar{\rho}_{\alpha}^{(\mathrm{II})} & \text { if } \mathbf{r} \text { is on side II, }\end{cases}$

which yields the modified PB equation for the total average electrostatic potential in the presence of a membrane potential $V_{\mathrm{mp}}$,

$\boldsymbol{\nabla} \cdot[\varepsilon(\mathbf{r}) \nabla \phi(\mathbf{r})]-\bar{\kappa}^{2}(\mathbf{r})\left[\phi(\mathbf{r})-V_{\mathrm{mp}} \Theta(\mathbf{r})\right]=-4 \pi \lambda \rho_{\mathrm{p}}(\mathbf{r})$.

We refer to this result as the linearized 'PB-V equation'. Closely related equations may be found in previous treatments of planar membranes (Läuger et al. 1967; Everitt \& Haydon, 1968; Walz et al. 1969). The PB-V Eq. (48) is equivalent to the standard PB Eq. (31) when $V_{\mathrm{mp}}=0$ and $\lambda=1$. The solution of the PB-V equation may be expressed formally as the sum of two separate terms

$\phi\left(\mathbf{r} ; V_{\mathrm{mp}}, \lambda\right)=V_{\mathrm{mp}} \phi_{\mathrm{mp}}(\mathbf{r})+\lambda \phi(\mathbf{r})$

with

$\phi_{\mathrm{mp}}(\mathbf{r})=-4 \pi \int \mathrm{d} \mathbf{r}^{\prime} G\left(\mathbf{r}, \mathbf{r}^{\prime}\right) \bar{\kappa}^{2}\left(\mathbf{r}^{\prime}\right) \Theta\left(\mathbf{r}^{\prime}\right)$

and

$\phi(\mathbf{r})=\int \mathrm{d} \mathbf{r}^{\prime} G\left(\mathbf{r}, \mathbf{r}^{\prime}\right) 4 \pi \rho_{\mathrm{p}}\left(\mathbf{r}^{\prime}\right)$,

where $G\left(\mathbf{r}, \mathbf{r}^{\prime}\right)$ is Green's function defined by

$\boldsymbol{\nabla} \cdot\left[\varepsilon(\mathbf{r}) \nabla G\left(\mathbf{r}, \mathbf{r}^{\prime}\right)\right]-\bar{\kappa}^{2}(\mathbf{r}) G\left(\mathbf{r}, \mathbf{r}^{\prime}\right)=-4 \pi \delta\left(\mathbf{r}-\mathbf{r}^{\prime}\right)$.

The function $\phi_{\mathrm{mp}}(\mathbf{r})$ multiplied by $V_{\mathrm{mp}}$ is the solution of Eq. (48) with $\lambda=0$, corresponding to the electrostatic potential due to the transmembrane voltage $V_{\mathrm{mp}}$ in the absence of the protein charges. The function $\phi_{\mathrm{mp}}$ is dimensionless with $0 \leqslant \phi_{\mathrm{mp}}(\mathbf{r}) \leqslant 1$ because of the properties of Eq. (48). It should be noted that $\phi_{\mathrm{mp}}(\mathbf{r})$ is independent of $V_{\mathrm{mp}}$ and of the protein charges (that formally are turned off when $\lambda=0$ ). However, it does depend on $\varepsilon(\mathbf{r})$ and $\bar{\kappa}^{2}(\mathbf{r})$. The function $\phi(\mathbf{r})$ is the solution of Eq. (48) with $V_{\mathrm{mp}}=0$, corresponding to the electrostatic potential due to the protein charges in the absence of any transmembrane voltage.

The electrostatic free energy of the microscopic system (relative to vacuum) is obtained from the reversible work required to build up the interactions in the system. The reversible work can be expressed as two successive thermodynamic integrations, first over $V_{\mathrm{mp}}$, and then over $\lambda$,

$\Delta G_{\text {elec }}\left(\mathbf{X}_{\mathrm{p}}\right)=\int_{0}^{V_{\mathrm{mp}}} \mathrm{d} V_{\mathrm{mp}} \int \mathrm{d} \mathbf{r} \Theta(\mathbf{r})\left\langle\rho_{\text {ions }}(\mathbf{r})\right\rangle_{\left(V_{\mathrm{mp}}, \lambda=0\right)}+\int_{0}^{1} \mathrm{~d} \lambda \sum_{p} q_{p}\left[V_{\mathrm{mp}} \phi_{\mathrm{mp}}\left(\mathbf{r}_{p}\right)+\lambda \phi_{\mathrm{rf}}\left(\mathbf{r}_{p}\right)\right]$, 
where $q_{p}$ and $\mathbf{r}_{p}$ are the protein charges and their position respectively, and $\phi_{\mathrm{rf}}$ is the reaction field defined as in Eq. (33). The complete result may be written in the form

$\Delta G_{\mathrm{elec}}\left(\mathbf{X}_{\mathrm{p}}\right)=\frac{1}{2} \mathcal{C} V_{\mathrm{mp}}^{2}+\left[\sum_{p} q_{p} \phi_{\mathrm{mp}}\left(\mathbf{r}_{p}\right)\right] V_{\mathrm{mp}}+\frac{1}{2} \sum_{p} q_{p} \phi_{\mathrm{rf}}\left(\mathbf{r}_{p}\right)$,

where $\mathcal{C}$ is the capacitance of the system (calculated with $\lambda=0$, in the absence of protein charges),

$\mathcal{C}=\int \mathrm{d} \mathbf{r} \Theta(\mathbf{r})\left(\frac{-\bar{\kappa}^{2}(\mathbf{r})}{4 \pi}\right)\left[\phi_{\mathrm{mp}}(\mathbf{r})-1\right]$.

The capacitance of the system depends very weakly on the configuration of the protein because $\varepsilon(\mathbf{r})$ and $\bar{\kappa}^{2}(\mathbf{r})$ may vary if the protein is not entirely embedded in the membrane region. In general, this contribution is negligible. The second term represents the interaction of the protein charges with the membrane potential. It may be expressed as $\mathcal{Q} V_{\text {mp }}$, where $\mathcal{Q}$ corresponds to an effective charge

$\mathcal{Q}=\sum_{p} q_{p} \phi_{\mathrm{mp}}\left(\mathbf{r}_{p}\right)$

The quantity $\phi_{\mathrm{mp}}\left(\mathbf{r}_{p}\right)$ represents the fraction of the membrane potential seen by each charge $q_{p}$. In the case of a perfectly planar system, the electric field across the membrane is constant and $\phi_{\mathrm{mp}}\left(\mathbf{r}_{p}\right)$ is simply the fraction of the membrane thickness. For this reason, it is often referred to as the 'electric distance' (Sigworth, 1993; Hille, 2001). This is illustrated in Fig. 6. More generally, the interaction of the protein charges with the membrane potential may be more complicated than the simple linear field if the shape of the protein-solution interface is irregular. The last term is independent of $V_{\mathrm{mp}}$ and corresponds to the self energy plus the reaction field contribution due to the solvent polarization and electrolyte shielding. This is sometime called the image interactions and electro-osmotic effect (Jordan et al. 1989). According to Eq. (34) the total configuration-dependent free energy $G\left(\mathbf{X}_{\mathrm{p}}\right)$ of the microscopic system can be written as

$G\left(\mathbf{X}_{\mathrm{p}}\right)=U_{\mathrm{p}}\left(\mathbf{X}_{\mathrm{p}}\right)+\Delta G_{\mathrm{np}}\left(\mathbf{X}_{\mathrm{p}}\right)+\Delta G_{\text {elec }}\left(\mathbf{X}_{\mathrm{p}}\right)$,

where $U_{\mathrm{p}}\left(\mathbf{X}_{\mathrm{p}}\right)$ is the internal potential energy of the system. The equilibrium probability of the system to be in the configuration $\mathbf{X}_{\mathrm{p}}$ is

$\mathcal{P}_{\text {eq }}\left(\mathbf{X}_{\mathrm{p}}\right)=\frac{\mathrm{e}^{-G\left(\mathbf{X}_{\mathrm{p}}\right) / k_{\mathrm{B}} T}}{\int \mathrm{d} \mathbf{X}_{\mathrm{p}}^{\prime} \mathrm{e}^{-G\left(\mathbf{X}_{\mathrm{p}}^{\prime}\right) / k_{\mathrm{B}} T}}$

which highlights the fact that the total configuration-dependent free energy, $G\left(\mathbf{X}_{\mathrm{p}}\right)$, is equivalent to a generalized macromolecular PMF. In analogy with Eq. (15), the total configuration-dependent free energy $G\left(\mathbf{X}_{\mathrm{p}}\right)$ can also be expressed as

$\mathrm{e}^{-G\left(\mathbf{X}_{\mathrm{p}}\right) / k_{\mathrm{B}} T}=\frac{\int \mathrm{d} \mathbf{X}^{\prime} \delta\left(\mathbf{X}_{\mathrm{p}}-\mathbf{X}_{\mathrm{p}}^{\prime}\right) \mathrm{e}^{-U_{\mathrm{tot}}\left(\mathbf{X}^{\prime}\right) / k_{\mathrm{B}} T}}{\int \mathrm{d} \mathbf{X}^{\prime} \delta\left(\mathbf{X}_{\mathrm{p}}-\mathbf{X}_{\mathrm{p}}^{\prime}\right) \mathrm{e}^{-U_{\mathrm{tot}}^{*}\left(\mathbf{X}^{\prime}\right) / k_{\mathrm{B}} T}}$,

where $U_{\text {tot }}^{*}$ corresponds to the potential energy of a reference system in which all interactions involving the microscopic subsystem have been turned off. This form will be useful in Section 4.3. 


\section{Statistical mechanical equilibrium theory}

The previous theoretical development provided a continuum approximation to the total free energy of a channel protein in a fixed configuration, embedded in a membrane and submitted to an applied transmembrane voltage. The expression for $G\left(\mathbf{X}_{\mathrm{p}}\right)$ remains valid, even if a number of ions and water molecules are considered as part of the microscopic system and treated explicitly, while the rest of the surrounding solvent is treated as a continuum. Nonetheless, important aspects of the molecular system have not been incorporated into the previous developments and further considerations are needed. In particular, the expression was developed in the context of approximations based on macroscopic continuum electrostatics. Even though some aspects of ions in aqueous solution (solvation free energy and ion-ion interaction) are well-described by continuum electrostatics, this approximation breaks down at length-scales shorter than 5-10. . The atomic nature of a flexible protein and the granularity of the water molecules should be taken into account for a meaningful representation of ion channels. Furthermore, several questions related to the properties of a pore system in open equilibrium with the bulk solutions need to be elucidated. For example, how many ions should partition into the pore region, and where should these ions bind in the pore is unresolved. In the following, we review the main elements of a statistical mechanical theory describing the equilibrium properties of ion channels initially formulated by Roux (1999a).

\section{I Multi-ion PMF}

Let us consider a selective ion channel embedded in a lipid membrane in equilibrium with surrounding aqueous salt solutions. The electrolyte solutions are not symmetric and there is a Nernst potential across the membrane. It is assumed that the channel is permeable to only one ionic species and remains in the open conducting state with no gating transitions; all other ions cannot pass through the channel or the membrane. It should be noted that ideal selectivity of the channel to one ionic species is a necessary condition for a true equilibrium situation to exist in the presence of asymmetric solutions.

The microscopic system is illustrated schematically in Fig. 8. The permeating ions can translocate from one side to the other, whereas the non-permeating ions cannot exchange from sides I and II. Since their number $N^{(\mathrm{S})}$ is fixed on each side S, their configurational integral is restricted to the side to which those ions are assigned. In contrast, the accessible configurational space of the permeating ions corresponds to the whole volume of the system. The total potential energy of the entire system is $U$ and $\mathbf{X}$ represents all the degrees of freedom in the system. More specifically, the coordinates of the $N$ permeable ions are indicated as $\left\{\mathbf{r}_{1}, \ldots, \mathbf{r}_{N}\right\}$.

To make progress, it is useful to define a 'pore' region from which all ions other than the permeating species are excluded, and a 'bulk' region which contains the electrolytic solutions. The step-functions $H_{\text {pore }}(\mathbf{r})$ and $H_{\text {bulk }}(\mathbf{r})$ are introduced such that $H_{\text {pore }}(\mathbf{r})=1$ when $\mathbf{r}$ is in the pore and 0 otherwise, and $H_{\mathrm{bulk}}(\mathbf{r})=1$ if $\mathbf{r}$ is in the bulk and 0 otherwise. By completeness, it follows that

$H_{\text {pore }}(\mathbf{r})+H_{\text {bulk }}(\mathbf{r}) \equiv 1$.

From this definition, it is possible to determine the total number of permeating ions inside the pore for any instantaneous configuration of the pore system. It is given by the discrete 


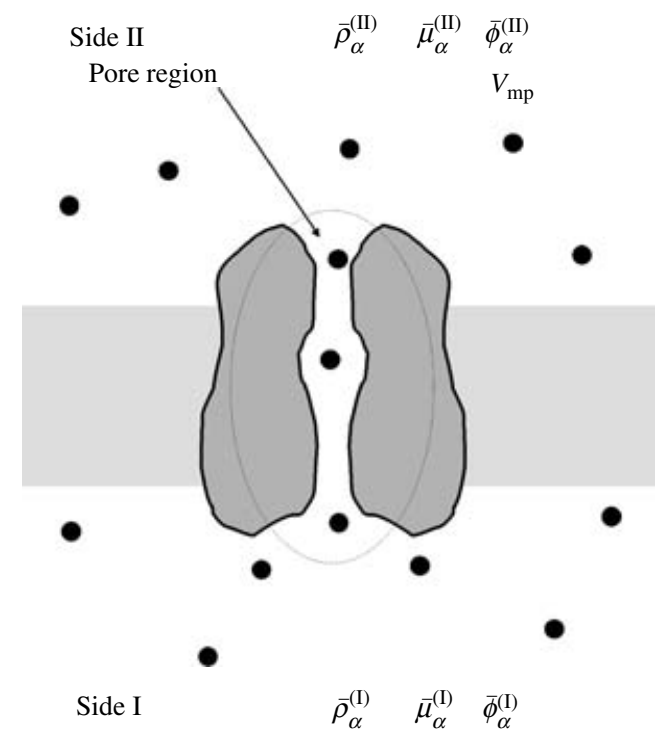

Fig. 8. Schematic representation of the ion channel-membrane system with asymmetrical solutions on sides I and II. The 'pore region', which corresponds to the ideally selective part of a channel, is highligted with a dashed line. The 'bulk region' corresponds to the remaining space in the system. As in Fig. 7, the transmembrane potential is 0 on side $\mathrm{I}$ and $V_{\mathrm{mp}}$ on side II. The system is assumed to be in thermodynamic equilibrium. The density of the permeable ions, the excess chemical potential, and the average electrostatic potential, are respectively, $\bar{\rho}^{(\mathrm{S})}, \bar{\mu}^{(\mathrm{S})}$ and $\bar{\phi}^{(\mathrm{S})}$ on side S (I or II) of the membrane. For any instantaneous configuration, it is possible to know the number of ions occupying the pore region.

function $n^{\prime}\left(\mathbf{r}_{1}, \mathbf{r}_{2}, \ldots, \mathbf{r}_{N}\right)$, defined as,

$n^{\prime}\left(\mathbf{r}_{1}, \mathbf{r}_{2}, \ldots, \mathbf{r}_{N}\right)=\sum_{i=1}^{N} H_{\text {pore }}\left(\mathbf{r}_{i}\right)$,

where $\mathbf{r}_{i}$ is the position of the $i$ th ion. The probability, $\mathcal{P}_{n}$, of having exactly $n$ ions inside the pore is calculated from the average,

$$
\begin{aligned}
\mathcal{P}_{n} & =\left\langle\delta_{n n^{\prime}}\right\rangle \\
& =\frac{\int \mathrm{d} \mathbf{X} \delta_{n, n^{\prime}} \mathrm{e}^{-U(\mathbf{X}) / k_{\mathrm{B}} T}}{\int \mathrm{d} \mathbf{X} \mathrm{e}^{-U(\mathbf{X}) / k_{\mathrm{B}} T}},
\end{aligned}
$$

where $\delta_{n n}^{\prime}$ is a Kroenecker function,

$\delta_{n n^{\prime}}= \begin{cases}1 & \text { if } n=n^{\prime}\left(\mathbf{r}_{1}, \mathbf{r}_{2}, \ldots, \mathbf{r}_{N}\right) \\ 0 & \text { otherwise }\end{cases}$

By construction, the probabilities $\mathcal{P}_{n}$ are normalized, i.e. $\Sigma_{n} \mathcal{P}_{n}=1$, via the completeness of the Kroenecker function. To determine the probabilities of occupancy, it is useful to consider the binding factor $\mathcal{B}_{n}$ corresponding to the ratio $\mathcal{P}_{n} / \mathcal{P}_{0}$. For $n=1$, this is

$\mathcal{B}_{1}=\frac{\int \mathrm{d} \mathbf{X} \delta_{1, n^{\prime}} \mathrm{e}^{-U(\mathbf{X}) / k_{\mathrm{B}} T}}{\int \mathrm{d} \mathbf{X} \delta_{0, n^{\prime}} \mathrm{e}^{-U(\mathbf{X}) / k_{\mathrm{B}} T}}$. 
Since the factor $\delta_{1, n}^{\prime}$ in the integrand is zero unless one of the $N$ ions is located inside the pore (only ion 1 could be in the pore, or only ion 2 , etc....), the expression may be re-written as

$\mathcal{B}_{1}=N \frac{\int \mathrm{d} \mathbf{X} H_{\text {pore }}\left(\mathbf{r}_{1}\right) H_{\text {bulk }}\left(\mathbf{r}_{2}\right) \cdots H_{\text {bulk }}\left(\mathbf{r}_{N}\right) \mathrm{e}^{-U(\mathbf{X}) / k_{\mathrm{B}} T}}{\int \mathrm{d} \mathbf{X} H_{\text {bulk }}\left(\mathbf{r}_{1}\right) H_{\text {bulk }}\left(\mathbf{r}_{2}\right) \cdots H_{\text {bulk }}\left(\mathbf{r}_{N}\right) \mathrm{e}^{-U(\mathbf{X}) / k_{\mathrm{B}} T}}$,

where the ion number 1 was chosen arbitrarily to occupy the pore. The pre-factor of $N$ is included to account for the multiple ways to obtain equivalent configurations. The 1 -ion binding factor may be expressed as

$\mathcal{B}_{1}=N \frac{\int \mathrm{d} \mathbf{r}_{1} H_{\text {pore }}\left(\mathbf{r}_{1}\right) \mathrm{e}^{-\mathcal{W}\left(\mathbf{r}_{1}\right) / k_{\mathrm{B}} T}}{\int \mathrm{d} \mathbf{r}_{1} H_{\text {bulk }}\left(\mathbf{r}_{1}\right) \mathrm{e}^{-\mathcal{W}\left(\mathbf{r}_{1}\right) / k_{\mathrm{B}} T}}$

where $\mathcal{W}\left(\mathbf{r}_{1}\right)$ is a PMF corresponding to the reversible thermodynamic work to adiabatically move one ion into the pore region (see Section 2.2 above). According to Eq. (66) the one-ion PMF is determined relative to an arbitrary offset constant. To have a simple relationship with the excess chemical potential of the ion in the bulk solution, we choose to define the PMF relative to a system with one non-interacting ion,

$\mathrm{e}^{-\mathcal{W}\left(\mathbf{r}_{1}\right) / k_{\mathrm{B}} T}=\frac{\int \mathrm{d} \mathbf{X}^{\prime} \delta\left(\mathbf{r}_{1}-\mathbf{r}_{1}^{\prime}\right) H_{\text {bulk }}\left(\mathbf{r}_{2}^{\prime}\right) \cdots H_{\text {bulk }}\left(\mathbf{r}_{N}^{\prime}\right) \mathrm{e}^{-U\left(\mathbf{X}^{\prime}\right) / k_{\mathrm{B}} T}}{\int \mathrm{d} \mathbf{X}^{\prime} \delta\left(\mathbf{r}_{1}-\mathbf{r}_{1}^{\prime}\right) H_{\text {bulk }}\left(\mathbf{r}_{2}^{\prime}\right) \cdots H_{\text {bulk }}\left(\mathbf{r}_{N}^{\prime}\right) \mathrm{e}^{-U\left(\mathbf{X}^{\prime} ; 1^{*}\right) / k_{\mathrm{B}} T}}$,

where the notation $U\left(\mathbf{X} ; 1^{*}\right)$ means that all interactions involving ion 1 with the rest of the system have been switched off (this potential energy is used as a reference system). It should be noted that, by construction, $\mathcal{W}\left(\mathbf{r}_{1}\right)$ is equal to the excess chemical potential $\bar{\mu}^{(\mathrm{S})}$ as $\mathbf{r}_{1}$ goes on side $\mathrm{S}$ far away from the pore region. The volume integral over the bulk region is

$$
\begin{aligned}
\int \mathrm{d}_{1} H_{\text {bulk }}\left(\mathbf{r}_{1}\right) \mathrm{e}^{-\mathcal{W}\left(\mathbf{r}_{1}\right) / k_{B} T} & =V^{(\mathrm{I})} \mathrm{e}^{-\mu^{(\mathrm{I}) / k_{B} T}}+V^{(\mathrm{II})} \mathrm{e}^{-\mu^{(\mathrm{II}) / k_{B} T}} \\
& =\frac{N}{\bar{\rho}^{(\mathrm{I})}} \mathrm{e}^{-\mu^{(\mathrm{I}) / k_{B} T}} \\
& =\frac{N}{\bar{\rho}^{(\mathrm{II})}} \mathrm{e}^{-\mu^{(\mathrm{II}) / k_{B} T}}
\end{aligned}
$$

because $N=V^{(\mathrm{I})} \bar{\rho}^{(\mathrm{I})}+V^{(\mathrm{II})} \bar{\rho}^{(\mathrm{II})}$ and $\mathrm{e}^{-\bar{\mu}^{(\mathrm{II})} / k_{\mathrm{B}} T}=\left(\bar{\rho}^{(\mathrm{II})} / \bar{\rho}^{(\mathrm{I})}\right) \mathrm{e}^{-\bar{\mu}^{(\mathrm{I}) / k_{\mathrm{B}} T}}$ according to Eq. (38). Since the density and the excess chemical potential at equilibrium are related via Eq. (39), it is possible to express the ratio $\mathcal{B}_{1}$ in terms of $\bar{\rho}^{(\mathrm{I})}$ or equivalently $\bar{\rho}^{(\mathrm{II})}$.

$$
\begin{aligned}
\mathcal{B}_{1} & =\bar{\rho}^{(\mathrm{I})} \int \mathrm{d} \mathbf{r}_{1} H_{\text {pore }}\left(\mathbf{r}_{1}\right) \mathrm{e}^{-\left[\mathcal{W}\left(\mathbf{r}_{1}\right)-\bar{\mu}^{(\mathrm{II})}\right] / k_{\mathrm{B}} T} \\
& =\bar{\rho}^{(\mathrm{II})} \int \mathrm{d} \mathbf{r}_{1} H_{\text {pore }}\left(\mathbf{r}_{1}\right) \mathrm{e}^{-\left[\mathcal{W}\left(\mathbf{r}_{1}\right)-\bar{\mu}^{(\mathrm{II})}\right] / k_{\mathrm{B}} T} .
\end{aligned}
$$

Similarly, the $n$-ion binding factor $\mathcal{B}_{n}$ is

$$
\mathcal{B}_{n}=\frac{N !}{n !(N-n) !} \times \frac{\int \mathrm{d} \mathbf{X} H_{\text {pore }}\left(\mathbf{r}_{1}\right) \cdots H_{\text {pore }}\left(\mathbf{r}_{n}\right) H_{\text {bulk }}\left(\mathbf{r}_{n+1}\right) \cdots H_{\text {bulk }}\left(\mathbf{r}_{N}\right) \mathrm{e}^{-U(\mathbf{X}) / k_{\mathrm{B}} T}}{\int \mathrm{d} \mathbf{X} H_{\text {bulk }}\left(\mathbf{r}_{1}\right) \cdots H_{\text {bulk }}\left(\mathbf{r}_{N}\right) \mathrm{e}^{-U(\mathbf{X}) / k_{\mathrm{B}} T}}
$$


since there are $N ! /(n !(N-n) !)$ equivalent configurations with identical ions. In the thermodynamic limit, $N \rightarrow \infty$ and the prefactor $N ! /(N-n) ! \approx N^{n}$, and

$\mathcal{B}_{n}=\left(\bar{\rho}^{(\mathrm{I})}\right)^{n} \frac{1}{n !} \int \mathrm{d} \mathbf{r}_{1} H_{\text {pore }}\left(\mathbf{r}_{1}\right) \cdots \int \mathrm{d} \mathbf{r}_{n} H_{\text {pore }}\left(\mathbf{r}_{n}\right) \mathrm{e}^{-\left[\mathcal{W}\left(\mathbf{r}_{1}, \ldots, \mathbf{r}_{n}\right)-n \bar{\mu}^{(I)}\right] / k_{\mathrm{B}} T}$,

where the $n$-ion PMF have been defined relative to $n$ non-interacting ions

$\mathrm{e}^{-\mathcal{W}\left(\mathbf{r}_{1}, \ldots, \mathbf{r}_{n}\right) / k_{\mathrm{B}} T}=\frac{\int \mathrm{d} \mathbf{X}^{\prime} \delta\left(\mathbf{r}_{1}-\mathbf{r}_{1}^{\prime}\right) \cdots \delta\left(\mathbf{r}_{n}-\mathbf{r}_{n}^{\prime}\right) H_{\text {bulk }}\left(\mathbf{r}_{n+1}^{\prime}\right) \cdots H_{\text {bulk }}\left(\mathbf{r}_{N}^{\prime}\right) \mathrm{e}^{-U\left(\mathbf{X}^{\prime}\right) / k_{\mathrm{B}} T}}{\int \mathrm{d} \mathbf{X}^{\prime} \delta\left(\mathbf{r}_{1}-\mathbf{r}_{1}^{\prime}\right) \cdots \delta\left(\mathbf{r}_{n}-\mathbf{r}_{n}^{\prime}\right) H_{\text {bulk }}\left(\mathbf{r}_{n+1}^{\prime}\right) \cdots H_{\text {bulk }}\left(\mathbf{r}_{N}^{\prime}\right) \mathrm{e}^{-U\left(\mathbf{X}^{\prime} ; 1^{*}, \ldots, n^{*}\right) / k_{\mathrm{B}} T}}$,

where the notation indicates that all interactions involving ion $1, \ldots, n$ have been switched off in the reference energy $U\left(\mathbf{X} ; 1^{*}, \ldots, n^{*}\right)$.

\subsection{Equilibrium probabilities of occupancy}

Once the binding factors $\mathcal{B}_{n}$ have been determined, the probability of any state of occupancy can be obtained using $\mathcal{B}_{0}=1$ with the normalization condition $\left\langle\delta_{n, n^{\prime}}\right\rangle=\left\langle\delta_{0, n^{\prime}}\right\rangle \mathcal{B}_{n}$, yielding,

$\mathcal{P}_{n}=\frac{\mathcal{B}_{n}}{1+\mathcal{B}_{1}+\mathcal{B}_{2}+\mathcal{B}_{3}+\cdots}$.

In particular, the probability that the pore be unoccupied is

$\mathcal{P}_{0}=\frac{1}{1+\mathcal{B}_{1}+\mathcal{B}_{2}+\mathcal{B}_{3}+\cdots}$.

The denominator in Eqs. (74) and (75) may be expressed in the form of an effective Grand Canonical Partition function of an open finite system in contact with a bath of particles,

$\Xi \equiv \sum_{n=0}^{\infty} \mathrm{e}^{n \bar{\mu}^{(\mathrm{I})} / k_{B} T}\left(\overline{\boldsymbol{\rho}}^{(\mathrm{I})}\right)^{n} \frac{1}{n !} \int \mathrm{d} \mathbf{r}_{1} H_{\text {pore }}\left(\mathbf{r}_{1}\right) \cdots \int \mathrm{d} \mathbf{r}_{n} H_{\text {pore }}\left(\mathbf{r}_{n}\right) \mathrm{e}^{-\mathcal{W}\left(\mathbf{r}_{1}, \ldots, \mathbf{r}_{n}\right) / k_{\mathrm{B}} T}$,

which provides a compact and useful notation for handling the multi-ion configurational distribution functions in the pore system. It follows that all equilibrium properties of the system can be rigorously reconstructed from a hierarchy of PMFs, $\mathcal{W}\left(\mathbf{r}_{1}, \ldots, \mathbf{r}_{n}\right)$, representing the pore region occupied by $n$ ions.

For any realistic channel, all the probabilities of occupancy $\mathcal{P}_{n}$ must be necessarily zero if $n$ is larger than some value $N_{\max }$, the maximum number of ions that can occupy the pore simultaneously. One important special case, the so-called 'one-ion' pore theory (Läuger, 1973; Levitt, 1986; McGill \& Schumaker, 1996), occurs if it is assumed that the pore cannot be occupied by more than one ion at a time. According to this assumption, it follows that all the binding factors $\mathcal{B}_{2}=\mathcal{B}_{3} \cdots=0$ and the probability of finding one ion inside the pore is simply,

$\mathcal{P}_{1}=\frac{\mathcal{B}_{1}}{1+\mathcal{B}_{1}}$.

This equation can be compared with the familiar rectangular hyperbola expression for first-order saturation of substrate binding

$\mathcal{P}_{1}=\frac{\bar{\rho}^{(\mathrm{I})} K_{1}}{1+\bar{\rho}^{(\mathrm{I})} K_{1}}$ 
where $K_{1}$ is the 1 -ion binding constant (expressed in terms of side I),

$K_{1}=\int \mathrm{d} \mathbf{r}_{1} H_{\text {pore }}\left(\mathbf{r}_{1}\right) \mathrm{e}^{-\left[\mathcal{W}\left(\mathbf{r}_{1}\right)-\bar{\mu}^{(\mathbb{I})}\right] / k_{\mathrm{B}} T}$.

The $n$-ion binding constants $K_{n}$ can be defined for multiply-occupied channels in a similar fashion. In general, the probability of multiply-occupied states, with explicit number of ions, can be expressed as

$\mathcal{P}_{n}=\frac{K_{n}\left(\overline{\boldsymbol{\rho}}^{(\mathrm{I})}\right)^{n}}{1+K_{1}\left(\overline{\boldsymbol{\rho}}^{(\mathrm{I})}\right)+K_{2}\left(\overline{\boldsymbol{\rho}}^{(\mathrm{I})}\right)^{2}+K_{3}\left(\overline{\boldsymbol{\rho}}^{(\mathrm{I})}\right)^{3}+\ldots}$.

The form of $\mathcal{P}_{n}$ is very similar to the familiar result, expressed as probability of multiplyoccupied states with explicit number of ions inside the pore which are obtained from kinetic models. Although such kinetic models are often formulated in terms of transition rate constants, the present results show that the equilibrium properties follow directly from a statistical mechanical analysis.

The hierarchy of constrained $n$-ion PMFs that was elaborated above is completely general and can be used to describe all equilibrium properties of a channel for any ionic concentrations. To describe the most general situation, the PMFs $\mathcal{W}\left(\mathbf{r}_{1}\right), \mathcal{W}\left(\mathbf{r}_{1}, \mathbf{r}_{2}\right), \mathcal{W}\left(\mathbf{r}_{1}, \mathbf{r}_{2}, \mathbf{r}_{3}\right)$, etc., are required. Each of these PMFs is defined on the basis of the pore function $H_{\text {pore }}(\mathbf{r})$, such that the number of explicit ions inside the pore region is exactly 1 , or 2 , or 3 , etc. In principle, the $n$-ion PMFs should be recalculated for different conditions of voltage and ion concentration. However, the dominant effect resulting from a change in ion concentration in the bulk is on the occupancy of the pore region, via the factors $\left(\bar{\rho}^{(\mathrm{I})}\right)^{n}$ in Eq. (80), rather than the $n$-ion PMFs themselves. In practice, the $n$-ion PMFs are expected to depend very weakly on the ionic concentration outside the pore region (see Section 6.1 .1 below). Nevertheless, for a correct description, the hierarchy of PMFs should not be truncated too early at some small number of explicit ions in the pore region if changes of concentration alter the occupancy of the pore significantly. For example, the gA channel is mostly singly occupied at low ion concentration but can be doubly occupied at moderately high concentration (Andersen \& Koeppe, 1992). In this case, a complete description valid for a wide range of concentration should include both the 1-ion and 2-ion PMFs.

\subsection{Coupling to the membrane potential}

So far, our treatment describes the most general situation with asymmetric solutions and a Nernst membrane potential. Symmetrical solutions with no membrane potential correspond to a particularly important special case. It may be anticipated that the equilibrium properties in the general situation can be expressed in terms of a dominant contribution corresponding to the symmetrical solutions with no membrane potential, plus other contributions associated with the transmembrane potential. For this purpose, we separate the system into pore and bulk subsystems and we represent all the degrees of freedom of the pore subsystem by $\mathbf{X}_{\mathrm{p}}$ (including ions, channels and water). One purpose of this separation is to enable us to use a continuum electrostatic approximation to describe the influence of the transmembrane potential on the pore subsystem. For the sake of simplicity, the present analysis will be carried for the 1-ion PMF although the treatment can easily be generalized to the $n$-ion PMFs described above using a similar route. 
The total free energy, $G$, of all the atoms of the pore subsystem in a fixed configuration $\mathbf{X}_{\mathrm{p}}$, allowing only ion 1 in the pore, is defined as

$\mathrm{e}^{-G\left(\mathbf{X}_{\mathrm{p}}\right) / k_{\mathrm{B}} T}=\frac{\int \mathrm{d} \mathbf{X}^{\prime} \delta\left(\mathbf{X}_{\mathrm{p}}-\mathbf{X}_{\mathrm{P}}^{\prime}\right) H_{\text {bulk }}\left(\mathbf{r}_{2}\right) \ldots H_{\text {bulk }}\left(\mathbf{r}_{N}\right) \mathrm{e}^{-U_{\text {tot }}\left(\mathbf{X}^{\prime}\right) / k_{\mathrm{B}} T}}{\int \mathrm{d} \mathbf{X}^{\prime} \delta\left(\mathbf{X}_{\mathrm{p}}-\mathbf{X}_{\mathrm{p}}^{\prime}\right) H_{\text {bulk }}\left(\mathbf{r}_{2}\right) \ldots H_{\text {bulk }}\left(\mathbf{r}_{N}\right) \mathrm{e}^{-U_{\mathrm{tot}}^{*}\left(\mathbf{X}^{\prime}\right) / k_{\mathrm{B}} T}}$,

Where $U_{\text {tot }}^{*}$ corresponds to the potential energy of a reference system in which all interactions in volving the pore subsystem have been turned off. A closed-form expression for the free energy $G\left(\mathbf{X}_{\mathrm{P}}\right)$ of a macromolecular subsystem in the membrane field was previously derived based on PB-V Eq. (48) [See Eq. (57) above]. One should note that the step-functions $H_{\text {bulk }}$ in Eq. (81) were not yet included in Eq. (59). Those are absolutely required in the definition of the 1-ion PMF to ensure that ions 2 to $n$ are restricted to the bulk region (see above). As a consequence, a modified ionic screening factor $\bar{\kappa}^{2}(\mathbf{r}) \rightarrow H_{\text {bulk }}(\mathbf{r}) \bar{\kappa}^{2}(\mathbf{r})$ must be used in the PB-V equation

$\boldsymbol{\nabla} \cdot[\varepsilon(\mathbf{r}) \boldsymbol{\nabla} \phi(\mathbf{r})]-H_{\text {bulk }}(\mathbf{r}) \bar{\kappa}^{2}(\mathbf{r})\left[\phi(\mathbf{r})-V_{\mathrm{mp}} \Theta(\mathbf{r})\right]=-4 \pi \rho_{\mathrm{p}}(\mathbf{r})$

to take this additional ion-exclusion constraint into account. In addition, the dielectric constant in the pore region should be set to 1 where the water molecules are represented explicitly (but see discussion below).

The free-energy function $G\left(\mathbf{X}_{\mathrm{p}}\right)$ generally depends on the density of ions on sides I and II, and on the transmembrane potential, i.e. $G\left(\mathbf{X}_{\mathrm{p}}\right)=G\left(\mathbf{X}_{\mathrm{p}} ; \bar{\rho}^{(\mathrm{I})}, \bar{\rho}^{(\mathrm{II})}, V_{\mathrm{mp}}\right)$. Let us define the free energy of the pore region under equilibrium symmetrical conditions, with no membrane potential as, $G_{\mathrm{eq}}\left(\mathbf{X}_{\mathrm{p}}\right) \equiv G\left(\mathbf{X}_{\mathrm{p}} ; \bar{\rho}^{(\mathrm{I})}=\bar{\rho}^{(\mathrm{II})}=\bar{\rho}, V_{\mathrm{mp}}=0\right)$. Following Eqs. (67) and (81), the 1-ion PMF may be expressed as

$\mathrm{e}^{-\mathcal{W}\left(\mathbf{r}_{1}\right) / k_{\mathrm{B}} T}=\frac{\int \mathrm{d} \mathbf{X}_{\mathrm{p}}^{\prime} \delta\left(\mathbf{r}_{1}-\mathbf{r}_{1}^{\prime}\right) \mathrm{e}^{-G\left(\mathbf{X}_{\mathrm{p}}^{\prime}\right) / k_{\mathrm{B}} T}}{\int \mathrm{d} \mathbf{X}_{\mathrm{p}}^{\prime} \delta\left(\mathbf{r}_{1}-\mathbf{r}_{1}^{\prime}\right) \mathrm{e}^{-G\left(\mathbf{X}_{\mathrm{p}}^{\prime} ; 1^{*}\right) / k_{\mathrm{B}} T}}$,

where the notation $G\left(\mathbf{X}_{p} ; 1^{*}\right)$ means that all interactions involving ion 1 with all atoms in the system (bulk and pore) have been switched off. In the general case, the 1-ion PMF $\mathcal{W}\left(\mathbf{r}_{1}\right)$ depends on all conditions influencing the system. Let us define the special case when there is no membrane potential, the system is at equilibrium and the PMF is $\mathcal{W}_{\text {eq }}\left(\mathbf{r}_{1}\right)$. We now seek an expression for the difference $\mathcal{W}\left(\mathbf{r}_{1}\right)-\mathcal{W}_{\text {eq }}\left(\mathbf{r}_{1}\right)$,

$$
\begin{aligned}
\mathrm{e}^{-\left[\mathcal{W}\left(\mathbf{r}_{1}\right)-\mathcal{W}_{\mathrm{eq}}\left(\mathbf{r}_{1}\right)\right] / k_{\mathrm{B}} T} & =\frac{\int \mathrm{d} \mathbf{X}_{\mathrm{p}}^{\prime} \delta\left(\mathbf{r}_{1}-\mathbf{r}_{1}^{\prime}\right) \mathrm{e}^{-G\left(\mathbf{X}_{p}^{\prime}\right) / k_{\mathrm{B}} T}}{\int \mathrm{d} \mathbf{X}_{\mathrm{p}}^{\prime} \delta\left(\mathbf{r}_{1}-\mathbf{r}_{1}^{\prime}\right) \mathrm{e}^{-G\left(\mathbf{X}_{p}^{\prime} ; 1^{*}\right) / k_{\mathrm{B}} T}} \times \frac{\int \mathrm{d} \mathbf{X}_{\mathrm{p}}^{\prime} \delta\left(\mathbf{r}_{1}-\mathbf{r}_{1}^{\prime}\right) \mathrm{e}^{-G_{\mathrm{eq}}\left(\mathbf{X}_{p}^{\prime} ; 1^{*}\right) / k_{\mathrm{B}} T}}{\int \mathrm{d} \mathbf{X}_{\mathrm{p}}^{\prime} \delta\left(\mathbf{r}_{1}-\mathbf{r}_{1}^{\prime}\right) \mathrm{e}^{-G_{\mathrm{eq}}\left(\mathbf{X}_{p}^{\prime}\right) / k_{\mathrm{B}} T}} \\
& =\frac{\int \mathrm{d} \mathbf{X}_{\mathrm{p}}^{\prime} \delta\left(\mathbf{r}_{1}-\mathbf{r}_{1}^{\prime}\right) \mathrm{e}^{-G\left(\mathbf{X}_{p}^{\prime}\right) / k_{\mathrm{B}} T}}{\int \mathrm{d} \mathbf{X}_{\mathrm{p}}^{\prime} \delta\left(\mathbf{r}_{1}-\mathbf{r}_{1}^{\prime}\right) \mathrm{e}^{-G_{\mathrm{eq}}\left(\mathbf{X}_{p}^{\prime}\right) / k_{\mathrm{B}} T}} \times \frac{\int \mathrm{d} \mathbf{X}_{\mathrm{p}}^{\prime} \delta\left(\mathbf{r}_{1}-\mathbf{r}_{1}^{\prime}\right) \mathrm{e}^{-G_{\mathrm{eq}}\left(\mathbf{X}_{p}^{\prime}, 1^{*}\right) / k_{\mathrm{B}} T}}{\int \mathrm{d} \mathbf{X}_{\mathrm{p}}^{\prime} \delta\left(\mathbf{r}_{1}-\mathbf{r}_{1}^{\prime}\right) \mathrm{e}^{-G_{\mathrm{eq}}\left(\mathbf{X}_{p}^{\prime} ; 1^{*}\right) / k_{\mathrm{B}} T}} \\
& =\left\langle\mathrm{e}^{-\Delta G / k_{\mathrm{B}} T}\right\rangle_{\left(\mathbf{r}_{1}\right)} \times\left\langle\mathrm{e}^{-\Delta G\left(1^{*}\right) / k_{\mathrm{B}} T}\right\rangle_{\left(\mathbf{r}_{1}^{*}\right)}^{-1},
\end{aligned}
$$

where $\Delta G=G-G_{\mathrm{eq}}$ and $\Delta G\left(1^{*}\right)=G\left(1^{*}\right)-G_{\mathrm{eq}}\left(1^{*}\right)$ are the excess perturbation free-energy contributions caused by asymmetrical conditions relative to symmetrical conditions relative to symmetrical systems. The bracket with subscript (eq, $\mathbf{r}_{1}$ ) represents an average,

$$
\langle\cdots\rangle_{\left(\mathbf{r}_{1}\right)} \equiv \frac{\int \mathrm{d} \mathbf{X}_{\mathrm{p}}^{\prime} \cdots \delta\left(\mathbf{r}_{1}-\mathbf{r}_{1}^{\prime}\right) \mathrm{e}^{-G_{\mathrm{eq}}\left(\mathbf{X}_{\mathrm{p}}^{\prime}\right) / k_{\mathrm{B}} T}}{\int \mathrm{d} \mathbf{X}_{\mathrm{p}}^{\prime} \delta\left(\mathbf{r}_{1}-\mathbf{r}_{1}^{\prime}\right) \mathrm{e}^{-G_{\mathrm{eq}}\left(\mathbf{X}_{\mathrm{p}}^{\prime}\right) / k_{\mathrm{B}} T}}
$$


with the ion fixed at $\mathbf{r}_{1}$, the configurations being Boltzmann-weighted by the free energy, $G_{\text {eq }}$, of a symmetrical equilibrium system. A similar expression holds for the configurational averages performed with the free energy $G_{\mathrm{eq}}\left(1^{*}\right)$ (in the latter case the subscript $\mathbf{r}_{1}$ can be dropped since averages are equivalent to those that would be calculated in the absence of ion inside the pore since its interactions have been switched off).

We must now evaluate the excess perturbation free energies, $\Delta G$ and $\Delta G\left(1^{*}\right)$. Their form is remarkably simple. The non-polar free-energy contribution, $G_{\mathrm{np}}$, can be ignored because it is independent of the membrane potential. Furthermore, even though the reaction-field freeenergy arises from long-range electrostatic interactions between the charges in the pore subsystem and the environment, it does not contribute to the excess perturbations if the ionic strength of the solution is kept unchanged on both sides of the membrane as the transmembrane potential is applied. It follows that

$$
\begin{aligned}
\Delta G\left(\mathbf{X}_{p} ; V_{\mathrm{mp}}\right) & =V_{\mathrm{mp}}\left[\sum_{p} q_{p} \phi_{\mathrm{mp}}\left(\mathbf{r}_{p}\right)\right] \\
& =V_{\mathrm{mp}}\left[q_{1} \phi_{\mathrm{mp}}\left(\mathbf{r}_{1}\right)+\sum_{p>1} q_{p} \phi_{\mathrm{mp}}\left(\mathbf{r}_{p}\right)\right],
\end{aligned}
$$

where particle 1 is the ion (the sum with $p>1$ runs over all particles other than the ion). Similarly,

$\Delta G\left(\mathbf{X}_{p} ; 1^{*}\right)=V_{\mathrm{mp}}\left[\sum_{p>1} q_{p} \phi_{\mathrm{mp}}\left(\mathbf{r}_{p}\right)\right]$

because the interactions of the ion with the surrounding are switched off. The function $\phi_{\mathrm{mp}}(\mathbf{r})$ represents the influence of the polarization of the counterions in the solvent at the membranebulk interface. It is calculated using Eq. (82) for a fixed configuration of the atoms in the pore region with all charges turned off. It is possible to treat the couplings $\Delta G$ and $\Delta G\left(1^{*}\right)$ perturbatively and express the complete PMF as a series in increasing powers of the membrane potential $V_{\mathrm{mp}}$. To this end, we develop the exact expression Eq. (84) in terms of a cumulant expansion (Balescu, 1975)

$\left\langle\mathrm{e}^{-\Delta G / k_{\mathrm{B}} T}\right\rangle=\mathrm{e}^{-\beta\langle\Delta G\rangle+\beta^{2}\left[\left\langle\Delta G^{2}\right\rangle-\langle\Delta G\rangle^{2}\right] / 2+\cdots}$

( $\beta=1 / k_{\mathrm{B}} T$ and the subscripts on the bracket have been omitted for the sake of clarity). To be valid, such a cumulant expansion does not require that the perturbation be small, but that the structure of the coupling with the reference system be simple. For example, it is rigorously exact if the fluctuations of the pore subsystem are Gaussian. From Eq. (84) the 1-ion PMF is, to lowest order in the perturbation,

$\mathcal{W}\left(\mathbf{r}_{1}\right)=\mathcal{W}_{\mathrm{eq}}\left(\mathbf{r}_{1}\right)+\mathcal{W}_{(1)}\left(\mathbf{r}_{1}\right)+\mathcal{W}_{(2)}\left(\mathbf{r}_{1}\right)+\cdots$,

where the first-order term is

$$
\begin{aligned}
\mathcal{W}_{(1)}\left(\mathbf{r}_{1}\right) & =\langle\Delta G\rangle_{\left(\mathbf{r}_{1}\right)}-\langle\Delta G\rangle_{\left(\mathbf{r}_{1}^{*}\right)} \\
& =V_{\mathrm{mp}}\left[q_{1} \phi_{\mathrm{mp}}\left(\mathbf{r}_{1}\right)+\left\langle\sum_{p>1} q_{\mathrm{p}} \phi_{\mathrm{mp}}\left(\mathbf{r}_{\mathrm{p}}\right)\right\rangle_{\left(\mathbf{r}_{1}\right)}-\left\langle\sum_{p>1} q_{p} \phi_{\mathrm{mp}}\left(\mathbf{r}_{p}\right)\right\rangle_{\left(\mathbf{r}_{1}^{*}\right)}\right]
\end{aligned}
$$


[the explicit expressions for $\Delta G$ and $\Delta G\left(1^{*}\right)$ have been used]. Thus, to linear order in $V_{\mathrm{mp}}$, the influence of the membrane potential arises from the interaction of all the charges of the pore subsystem (i.e. the ion, the channel and its water content) with $\phi_{\mathrm{mp}}$. The second-order term (quadratic in $V_{\mathrm{mp}}$ ) is related to fluctuations. Because the ion is fixed at $\mathbf{r}_{1}$, the direct term $q_{1} \phi_{\mathrm{mp}}\left(\mathbf{r}_{1}\right)$ does not contribute to the fluctuations, although its presence has a direct influence on the magnitude of the fluctuations of the other components (water and protein) inside the pore. Although the coupling of the pore region with the transmembrane potential has been detailed for the case of a 1-ion PMF, the expressions in Eqs. (81)-(89) are easily generalized for multi-ion PMFs.

One could use the present development representing the multi-ion PMFs in a pore region and its coupling to the transmembrane potential in different ways, depending on the ion channel. The equilibrium PMF corresponds to a thermal average for the molecular system under equilibrium conditions in which all the microscopic interactions (i.e. ion-channel, ion-water and water-channel) are expected to play an essential role. In the case of wide aqueous pores, an allatom MD approach may not be practical and it may be necessary to calculate the $n$-ions PMFs assuming that the solvent is represented by a continuum dielectric in all aqueous regions. In this case, the total PMF can be calculated directly on the basis of PB-V Eq. (82) using the closedform expression Eq. (57) previously derived for the free energy $G\left(\mathbf{X}_{\mathrm{p}}\right)$ of a macromolecular subsystem embedded in a membrane. This approximation is elaborated in more detail in Section 5.2.3 in the context of the GCMC/BD algorithm. Nonetheless, the equilibrium PMF is expected to be sensitive to the structural flexibility of the protein and the granularity of the water molecules near the ions, particularly in the case of narrow pores such as gA or KcsA. At such lengthscales, a continuum dielectric representation of the water molecules in the pore is not valid (see Section 3.1 above). For narrow pores, meaningful estimates of the relevant equilibrium PMFs can be obtained only using all-atom MD simulations including explicit water molecules (see Section 5.1.2 below). It may be possible, then, to use all-atom MD simulations to compute all the averages required for the cumulant expansion to represent the coupling of the pore system to the transmembrane potential (Allen et al. 2003b). However, calculating the cumulant averages $\mathcal{W}_{(1)}\left(\mathbf{r}_{1}\right)$ and $\mathcal{W}_{(2)}\left(\mathbf{r}_{1}\right)$ required in Eq. (89) becomes difficult when there is a wide vestibule containing many water molecules, such as in the case of the KcsA channel. Nevertheless, the coupling with the transmembrane potential is expected to be reasonably well-represented by a continuum dielectric approximation because it is essentially electrostatic in nature. This suggests an intermediate approach in which the equilibrium PMF is calculated from all-atom MD but the coupling to the transmembrane potential is calculated assuming that the solvent in the pore is represented by a continuum dielectric (Bernèche \& Roux, 2003),

$\mathcal{W}_{\mathrm{mp}}\left(\mathbf{r}_{1}, \mathbf{r}_{2}, \ldots\right)=V_{\mathrm{mp}} \sum_{i} q_{i} \phi_{\mathrm{mp}}\left(\mathbf{r}_{i}\right)$,

where $\phi_{\mathrm{mp}}$ is calculated by solving PB-V Eq. (82) with no explicit water molecules (i.e. the space-dependent dielectric constant $\varepsilon(\mathbf{r})$ is set to $\left.\varepsilon_{\mathrm{w}}\right)$. Accordingly, the total PMF is simply expressed as

$\mathcal{W}\left(\mathbf{r}_{1}, \mathbf{r}_{2}, \ldots\right)=\mathcal{W}_{\mathrm{eq}}\left(\mathbf{r}_{1}, \mathbf{r}_{2}, \ldots\right)+V_{\mathrm{mp}} \sum_{i} q_{i} \phi_{\mathrm{mp}}\left(\mathbf{r}_{i}\right)$.

One may note that, in this case, the fluctuations corresponding to the second cumulant are implicitly incorporated via the dielectric response of the solvent and the expansion in Eq. (89) is 
limited to the first cumulant. For the sake of clarity, one should distinguish between the effect of the transmembrane potential when it is calculated using the PB-V equation and averages in the cumulant expansion extracted from all-atom MD, or when it is calculated as in Eq. (91) using the PB-V equation, but representing the solvent inside the pore as a continuum dielectric. For the sake of clarity, we shall refer to the former as $V_{\mathrm{mp}}(\mathrm{PB}-\mathrm{V}, \mathrm{MD})$, and to the latter as $V_{\mathrm{mp}}(\mathrm{PB}-\mathrm{V})$ (see also Table 1$)$.

\section{4 lonic selectivity}

The selectivity of an ion channel reflects the difficulty that an 'undesired' ion type has to replace a 'desired' ion type. One should distinguish selectivity arising from equilibrium ion binding versus selectivity arising from kinetic and non-equilibrium processes (Andersen \& Koeppe, 1992). Furthermore, selectivity may manifest itself very differently, depending on experimental conditions or the properties of a particular channel. For example, the cation-selective OmpF, like most porins, exhibits only a very modest charge specificity (Cowan et al. 1992; Schirmer, 1998). Such modest selectivity in the case of $\mathrm{OmpF}$ is not surprising given that the permeation pathway is a wide aqueous pore and that the permeation process involves ion and counterions ( $\mathrm{Im} \&$ Roux, 2002a). In contrast, KcsA, like most biological $\mathrm{K}^{+}$channels, is very selective for $\mathrm{K}^{+}$over $\mathrm{Na}^{+}$(Heginbotham et al. 1999; LeMasurier et al. 2001; Nimigean \& Miller, 2002). The gA channel, exhibits a somewhat intermediate selectivity, is very specific for the small univalent cations $\mathrm{H}^{+}, \mathrm{Li}^{+}, \mathrm{Na}^{+}, \mathrm{K}^{+}, \mathrm{Rb}^{+}$, and $\mathrm{Cs}^{+}$, but is essentially impermeable to anions and divalent cations (Andersen \& Koeppe, 1992). The modest selectivity of porins is best represented in terms of the reversal potential at zero net current in asymmetric salt solution. In the case of $\mathrm{gA}$ or KcsA, where the ion permeation process involves a small number of well-defined states of ion occupancy, it may be expected that ion selectivity arises largely from the properties of the equilibrium PMF. To make progress, let us consider the permeation of one ion of type $\eta$ through a channel that is very selective for ions of type $\alpha$. A quantity of particular interest is the freeenergy difference needed to replace a single ion of type $\alpha$ by an undesired ion of type $\eta$ at point r. According to Eq. (73)

$\mathrm{e}^{-\left[\mathcal{W}_{\eta}(\mathbf{r})-\mathcal{W}_{\alpha}(\mathbf{r})\right] / k_{\mathrm{B}} T}=\frac{\int \mathrm{d} \mathbf{X}^{\prime} \delta\left(\mathbf{r}-\mathbf{r}^{\prime}\right) \mathrm{e}^{-U_{\eta}(\mathbf{X}) / k_{\mathrm{B}} T}}{\int \mathrm{d} \mathbf{X}^{\prime} \delta\left(\mathbf{r}-\mathbf{r}^{\prime}\right) \mathrm{e}^{-U_{\alpha}(\mathbf{X}) / k_{\mathrm{B}} T}}$,

where the previous notation has been simplified for the sake of clarity. Expressions such as Eq. (93) are naturally evaluated using the techniques of alchemical FEP (McCammon \& Straatsma, 1992; Kollman, 1993),

$\mathrm{e}^{-\left[\mathcal{W}_{\eta}(\mathbf{r})-\mathcal{W}_{\alpha}(\mathbf{r})\right] / k_{\mathrm{B}} T}=\left\langle\mathrm{e}^{-\left[U_{\eta}-U_{\alpha}\right] / k_{\mathrm{B}} T}\right\rangle_{(\alpha, \mathrm{r})}$,

where the bracket implies an average from an ensemble of equilibrium configurations with an ion of type $\alpha$ held fixed at point $\mathbf{r}$. According to Eq. (79), the occupancy of the pore is governed by the relative free energy of the two ions in the bulk and in the channel

$\Delta \Delta \mathcal{W}_{\eta-\alpha}=\left[\mathcal{W}_{\eta}(\mathbf{r})-\bar{\mu}_{\eta}\right]-\left[\mathcal{W}_{\alpha}(\mathbf{r})-\bar{\mu}_{\alpha}\right]$ 
More generally, the same FEP technique can be used to assess the selectivity in a multi-ion pore by considering the PMF difference between a pore occupied by different numbers of ions of type $\alpha$ and $\eta$. For example, the selectivity of the KcsA $\mathrm{K}^{+}$channel can be assessed by calculating the free energy required to alchemically transform a single $\mathrm{K}^{+}$into a $\mathrm{Na}^{+}$ion, while the other $\mathrm{K}^{+}$ions in the pore are not transformed (Allen et al. 1999, 2000; Aqvist \& Luzhkov, 2000; Bernèche \& Roux, 2001; Luzhkov \& Åqvist, 2001a).

\subsection{Reduction to a one-dimensional (ID) free-energy profile}

As shown in the previous section, a hierarchy of $n$-ion PMFs in $3 \mathrm{D}$ space arises naturally from a rigorous statistical mechanical formulation of equilibrium properties. However, ion permeation is traditionally discussed in terms of the free-energy profile of ions along the channel axis (Hille, 2001). In particular, such quantity is an essential input to simple kinetic rate models (Läuger, 1973) and in the 1D Nernst-Planck (1D-NP) electrodiffusion equation (Levitt, 1986; McGill \& Schumaker, 1996). From a dynamical point of view, the reduction in dimensionality from $\mathbf{r}$ to $z$ is based on the assumption that all motions perpendicular to $z$ reach equilibrium rapidly and that $z$ is the only relevant slow variable in the system, i.e. it can be chosen as a meaningful reaction coordinate (see the discussion in Section 2.1). This assumption seems reasonable for narrow channels, such as gA of KcsA, although even in those cases small systematic departures from the main axis can also play an important role in ion dynamics (e.g. see Roux \& Karplus, 1991a; Elber et al. 1995). Nevertheless, the 1D free-energy profile is a useful concept and for this reason, it is worthwhile examining its microscopic significance. The $1 \mathrm{D}$ free-energy profile, $\mathcal{W}_{1 \mathrm{D}}(z)$ is formally defined as

$\mathrm{e}^{-\left[\mathcal{W}_{1 \mathrm{D}}(\vartheta)-\mathcal{W}_{1 \mathrm{D}}\left(z_{\mathrm{ref}}\right)\right] / k_{\mathrm{B}} T}=C \frac{\int \mathrm{d} x \int \mathrm{d} y \mathrm{e}^{-\mathcal{W}_{3 \mathrm{D}}(x, y, z) / k_{\mathrm{B}} T}}{\int \mathrm{d} x \int \mathrm{d} y \mathrm{e}^{-\mathcal{W}_{3 \mathrm{D}}\left(x, y, \text { ref }_{\mathrm{ref}}\right) / k_{\mathrm{B}} T}}$,

where $C$ is an arbitrary constant and $\mathcal{W}_{3 \mathrm{D}}(x, y, z)$ is the complete PMF in $3 \mathrm{D}$ and $z_{\text {ref }}$ is some reference position (for the sake of clarity, subscripts $1 \mathrm{D}$ and $3 \mathrm{D}$ on the PMFs will be used explicitly for the present development). Obviously, such integration has significance only in the pore region, where the lateral displacements of the ion are bounded. Far away from the channel, the 1D free-energy profile is mathematically ill-defined and can actually diverge because the ion has infinitely more lateral freedom in the bulk region than inside the pore. Furthermore, because the constant $C$ is arbitrary, only the relative variations of the $1 \mathrm{D}$ free profile are meaningful. Consequently, there is no unique way to assign a value to $\mathcal{W}_{1 \mathrm{D}}(z)$ with respect to some absolute free-energy scale [e.g. it is incorrect to assume that $\mathcal{W}_{1 \mathrm{D}}(z)$ must be equal to zero for large $z$, where the ion is in the bulk region and its lateral motions are not bounded]. One convenient choice for the normalization of the 1D free-energy profile is

$\mathrm{e}^{-\mathcal{W}_{1 \mathrm{D}}(\vartheta) / k_{\mathrm{B}} T} \equiv \mathrm{e}^{-\left[\mathcal{W}_{3 \mathrm{D}}\left(0,0, \psi_{\mathrm{ref}}\right)-\bar{\mu}^{(\mathrm{I})}\right] / k_{\mathrm{B}} T} \frac{\int \mathrm{d} x \int \mathrm{d} y \mathrm{e}^{-\mathcal{W}_{3 \mathrm{D}}(x, y, y) / k_{\mathrm{B}} T}}{\int \mathrm{d} x \int \mathrm{d} y \mathrm{e}^{-\mathcal{W}_{3 \mathrm{D}}\left(x, y, \gamma_{\mathrm{ref}}\right) / k_{\mathrm{B}} T}}$,

where $\bar{\mu}^{(\mathrm{I})}$ is the excess chemical potential far away from the channel in the bulk region. A unique definition of the cross-section of the pore, $\mathrm{S}$, is consistent with this definition of the 
1D free-energy profile,

$S=\int \mathrm{d} x \int \mathrm{d} y \mathrm{e}^{-\left[\mathcal{W}_{3 \mathrm{D}}(x, y, \text { ref })-\mathcal{W}_{3 \mathrm{D}}(0,0, \text { r ref }) / k_{\mathrm{B}} T\right.}$.

This definition is very convenient to establish a link with well-known expressions for the 1-ion equilibrium binding constant in terms of the free-energy profile and the cross-sectional area of the channel (Levitt, 1986)

$K_{1}=S \int_{\text {pore }} \mathrm{d} z \mathrm{e}^{-\mathcal{W}_{1 \mathrm{D}}(\eta) / k_{\mathrm{B}} T}$

which is rigorously equivalent to Eq. (79). As defined by Eq. (98), the cross-sectional area of the pore is related the amount of lateral fluctuations that the ion position can undergo at some prescribed position zref along the axis of the channel. Based on this analysis, a single value is defined for the cross-sectional area of the pore. The definition can then be used with the definition of the 1D free-energy profile given by Eq. (97) to yield the correct binding constant via Eq. (79). This way of defining $S$ is quite convenient for a channel that does not have large variations in geometry and width (e.g. gA or the selectivity filter of KcsA). But it may not particular useful for describing a wide pore such as OmpF. Alternatively, one can choose to consider a cross-sectional area $\mathrm{S}(z)$ that varies along the channel axis (Smart et al. 1993). But then, one must be careful to combine $\mathrm{S}(z)$ with a $1 \mathrm{D}$ free-energy profile. In particular, it would be incorrect to compute a $1 \mathrm{D}$ free-energy profile $W_{1 \mathrm{D}}(z)$ for a channel using umbrella sampling, then compute the $z$-dependent cross-sectional area $S(z)$, and finally incorporate these two quantities into some BD or NP model to simulate ion permeation. The $1 \mathrm{D}$ free-energy profile includes the variations of lateral freedom of the ion, i.e. $k_{\mathrm{B}} T \ln \left(\mathrm{S}(z) / \mathrm{S}_{\text {ref }}\right)$, and those should not be double counted by the cross section.

\section{From MD to I-V: a practical guide}

The previous sections (2-4) have presented a very formal perspective on the ion permeation. In the first part of this section, we will review some of the special computational techniques that can be used to extract key information from all-atom MD simulations. We will first summarize fundamental results from linear response theory and relate the conductance of a channel to the fluctuations observed during unbiased equilibrium MD trajectories. We will also briefly discuss how the conductance can be estimated from non-equilibrium MD trajectories. We will then review the methods for calculating the PMF and the diffusion coefficient of ions inside a channel using biased MD simulations. This first part ends with a brief discussion of various issues concerning computational efficiency using different approaches.

In the second part of this section, we will briefly go over a number of permeation models that can be used to calculate ion fluxes. We will review the single-ion NP model (Lewitt, 1986; McGill \& Schumaker, 1996), which is of particular interest because it can be solved analytically. We will also describe framework models based on continuous time discrete-state Markov chains, which can handle multi-ion pores (McGill \& Schumaker, 1996; Schumaker et al. 2000, 2001; Bernèche \& Roux, 2003). Such framework models are the most convenient and powerful approaches to incorporate the information extracted from MD simulations, such as the PMF 
and the diffusion coefficient. We will then review the GCMC/BD algorithm (Im et al. 2000) and the PNP electrodiffusion theory for simulating ion flow through wide pores.

\section{I Extracting the essential ingredients from $M D$}

\section{I.I Channel conductance from equilibrium and non-equilibrium MD}

According to the fluctuation-dissipation theorem (Kubo, 1966), all the information required to estimate the near-equilibrium conductance of an ion channel at small voltage is, in principle, included in the fluctuations observed during straight unbiased all-atom equilibrium MD simulations. Expressions for transport coefficients can be derived using the formalism of linear response theory (Kubo, 1966; McQuarrie, 1976). Those expressions have typically the form of a time correlation function that must be evaluated from a system at equilibrium. To establish the fundamental expression for the conductance of an ion channel, let us consider an external electric circuit connected via two chemiabsorption electrodes to a membrane-channel system surrounded by aqueous salt solutions. An EMF controls exactly the relative potential between the two electrodes. The instantaneous amount of electrical current flowing through the external circuit is $\dot{Q}_{\text {ext }}(t)$. It is assumed that the resistance of the external circuit is negligible and that the exact detail of the chemiabsorption process is unimportant. When a voltage of zero is imposed, there is no net current on average and $\left\langle\dot{Q}_{\text {ext }}(t)\right\rangle_{(\mathrm{eq})}=0$. At time $t=0$, a small potential step $V_{\mathrm{mp}}$ is applied giving rise to a non-equilibrium perturbation. After a long time, the average current reaches a stationary state and the system obeys Ohm's law, $I=g V_{\mathrm{mp}}$, where the conductance $g$ is given by

$g=\frac{1}{k_{\mathrm{B}} T} \int_{0}^{\infty} \mathrm{d} t\left\langle\dot{Q}_{\text {ext }}(t) \dot{Q}_{\text {ext }}(0)\right\rangle_{(\text {eq })}$

according to linear response theory (Kubo, 1966; McQuarrie, 1976). The relationship of the conductance of the system and the current autocorrelation function, which was first discovered by Nyquist (1928), is a realization of the fluctuation-dissipation relation for an electrical circuit. Following the general properties of flux-flux correlation functions (Helfand, 1960), one can conveniently re-express Eq. (100) as

$g=\lim _{t \rightarrow \infty} \frac{1}{k_{\mathrm{B}} T} \frac{\left\langle\left|Q_{\mathrm{ext}}(t)-Q_{\mathrm{ext}}(0)\right|^{2}\right\rangle_{(\mathrm{eq})}}{2 t}$.

It may be noted that the charge $Q_{\text {ext }}(t)$ in Eq. (101) plays a role similar to that of the position during a random walk. This analysis shows how, to linear order in the perturbation potential, the conductance of the system is fundamentally related to the thermal fluctuations in the charge movements through the external circuit. Equation (101) can be used to relate the magnitude of the channel conductance to the number of ions observed to randomly move across a channel during an equilibrium MD trajectory. Let us suppose that $\mathcal{N}$ complete ion-crossing events (forward and/or backward) are observed during a trajectory of length $\tau$. Assuming that the crossing events are independent and can be represented as a Poisson process with an average number of crossings per unit of time of $\mathcal{N} / \tau$, the estimated conductance is simply

$g \approx \frac{\mathcal{N} q^{2}}{2 \tau k_{\mathrm{B}} T}$ 
Equation (102) provides a direct relation between the number of independent ions observed to randomly move across an ion channel during an equilibrium trajectory, and the channel's conductance.

Alternatively, it is conceivable to monitor the net average current during non-equilibrium trajectory in the presence of an applied external transmembrane potential $V_{\mathrm{mp}}$ (Suenaga et al. 1998; Crozier et al. 2001a, b; Yang et al. 2003). The channel conductance can then be estimated from the average number of crossings per unit of time

$g \approx \frac{\mathcal{N} q}{\tau V_{\mathrm{mp}}}$.

Although this would appear to be the most obvious approach to calculating the conductance of an ion channel, it is not as straightforward as it appears. In particular, it is important to ensure that the external field be introduced in a physically realistic and meaningful way. The simplest approach to simulate a transmembrane potential in MD consists in applying a constant electric field acting on all the charges in the system along the axis perpendicular to the membrane bilayer (Crozier et al. 2001a, b; Tieleman et al. 2001a; Yang et al. 2003). By virtue of the periodic boundary condition, the electrostatic potential is discontinuous at the boundary of the simulation system though the electric field and the forces acting on all the charges are continuous. Nonetheless, the significance of such an approach might require further clarification to relate the externally applied constant electric field to the true physical transmembrane potential, that arises from a small charge imbalance and a polarization of the membrane-solution interface (see Section 3.6). One might be able to relate the constant external electric field to the polarization interface in regions of space that are far away from the simulation system, in a spirit similar to the treatment of boundary conditions in the Ewald lattice sum (DeLeeuw et al. 1980).

\subsubsection{PMF techniques}

The multi-ion PMF, which corresponds to the reversible work by the average microscopic forces arising in the complex environment of the pore is, perhaps, the most important quantity in trying to understand ion permeation. As shown in Section 2.2, all equilibrium properties of the multiion pore are completely determined by the equilibrium PMF. Much of Section 4 was dedicated to the clarification of the detailed formulation of multi-ion PMFs and the coupling to the transmembrane potential in the context of ion channels. Although such a discussion of the PMF at an abstract conceptual level is needed to define these fundamental ideas, it is necessary to calculate the equilibrium PMF to proceed any further. In practice, all-atom MD is the only approach that can be used to calculate a meaningful PMF that incorporates the influence of all known microscopic factors (e.g. fluctuations of the protein channel and solvation by discrete water molecules). We shall refer to such an equilibrium PMF as PMF(MD). When the reaction coordinate is a simple Cartesian coordinate, e.g. the position $z$ of an ion along the channel axis, the PMF can be calculated using holonomic constraints (stringent geometric conditions) and the free-energy simulation technique (Zwanzig, 1954). The PMF at a position $\mathcal{W}(z+\Delta z)$ can be expressed in terms of $\mathcal{W}$ (z) (Tobias \& Brooks, 1987; Roux \& Karplus, 1991b),

$$
\begin{aligned}
\Delta \mathcal{W}(z \rightarrow z+\Delta z) & =\mathcal{W}(z+\Delta z)-\mathcal{W}(z) \\
& =-k_{\mathrm{B}} T \ln \left\langle\mathrm{e}^{-\Delta U / k_{\mathrm{B}} T}\right\rangle_{(z)},
\end{aligned}
$$


where the parentheses with subscript $z$ represents a thermal average with the ion coordinate held fixed at $z$ and $\Delta U$ is the change in potential energy obtained by displacing the coordinate from $z$ to $z+\Delta z$ (but keeping all other coordinates unchanged). It should emphasized that the $x$ and $y$ coordinate of the ion are allowed to move during the sampling trajectory. The average in Eq. (104) is calculated from an ensemble of configurations generated by a computer simulation of the system in thermal equilibrium with the ion coordinate fixed at $z$ using a holonomic constraint. In practice it is necessary to generate several trajectories of the system with the ion coordinate fixed at different values of $z$ and the complete profile is constructed by joining the free energy differences obtained with Eq. (104). The PMF may also be calculated by integrating the reversible work done by the mean force $\langle F(z)\rangle$ acting on the ion in the z direction

$\mathcal{W}(z)=\mathcal{W}\left(z_{0}\right)-\int_{z_{0}}^{z} \mathrm{~d} z^{\prime}\left\langle F\left(z^{\prime}\right)\right\rangle$

An advantage of this formulation is that the mean force can be decomposed linearly into a sum of contributions (Roux \& Karplus, 1991b; Allen et al. 2003b). However, it is difficult to apply this approach to multi-dimensional cases. Another method to compute the PMF is the 'umbrella sampling' technique (Torrie \& Valleau, 1974; Valleau \& Torrie, 1977). In this method, the microscopic system of interest is simulated in the presence of an imposed biasing window potential, $u_{i}(z)$, introduced to enhance the sampling in the vicinity of a chosen value $z$. The biased simulations are generated using the potential energy, $U(\mathbf{X})+u_{i}(\mathrm{z})$. Typically, the biasing potential serves to confine the variations of the coordinate $z$ within a small interval around some prescribed value $z$, helping to achieve a more efficient configurational sampling in this region (this is the reason why the biasing potential is called a window potential). For example, a reasonable choice to produce the biased ensembles, though not the unique one, is to use harmonic functions of the form, $u_{i}(z)=\frac{1}{2} K\left(z-z_{i}\right)^{2}$, centered on successive values of $z_{i}$. Because the sampling is confined to a small region during a given biased simulation, only a small piece of the estimated PMF is sufficiently accurate to be useful. The piece of unbiased PMF from the $i$ th window is,

$\mathcal{W}_{i}(z)=F_{i}-k_{\mathrm{B}} T \ln \left[\langle\rho(z)\rangle_{(i)}^{(\text {biased })}\right]-u_{i}(z)$,

where $\langle\rho(z)\rangle_{(i)}^{(\text {biased })}$ is the biased histogram from the $i$ th simulation and $F_{i}$ is an undetermined free energy constant. To obtain the PMF over the whole range of interest of $z$ it is necessary to perform a number of biased window simulations, each biasing the configurational sampling around a different region of $\%$. To obtain the complete PMF, the bias introduced by the constraining potential has to be removed and the data from several windows have to be combined together. The most efficient procedure to do this is the weighted histogram analysis method (WHAM) (Kumar et al. 1992). One of the main advantages of WHAM is that it can be easily extended to treat the case of a PMF depending on more than one variable (Boczko \& Brooks, 1993; Roux, 1995), e.g. Bernèche \& Roux (2001) calculated a 3D PMF depending on the $Z$ position of $3 \mathrm{~K}^{+}$in KcsA and Smith \& Sansom (2002) calculated a 3D PMF for a single ion going through the Alm channel.

\section{I.3 Friction and diffusion coefficient techniques}

The multi-ion PMF, which relates to the average and systematic forces arising in the environment of the pore, is not sufficient to completely characterize ion permeation. The random 
chaotic movements of ions through a molecular pore is also affected by processes that are intrinsically dynamical and dissipative. Effective dynamical models, such as the GLE, LE or BD incorporate the influence of such factors into the memory function, friction coefficient $\gamma$, or diffusion coefficient $D$. The ion diffusion coefficient at different position inside the pore is a particularly important parameter in a number of theoretical models of permeation (Chiu et al. 1993; McGill \& Schumaker, 1996; Kurnikova et al. 1999; Smith \& Sansom, 1999; Im et al. 2000; Allen \& Chung, 2001; Im \& Roux, 2001). The diffusion coefficient is often defined in terms of the average mean-square displacement (MSD) (Einstein, 1926),

$D=\lim _{t \rightarrow \infty} \frac{\left\langle[z(t)-z(0)]^{2}\right\rangle}{2 t}$

where the time, $t$, has to be large compared to the relaxation times of microscopic processes in the solution. Though this expression is rigorously valid only for a isotropic uniform environment, it has been used to calculate the space-dependent diffusion coefficient $D(z)$ of freely moving ions and water molecules in transmembrane pores (Tieleman \& Berendsen, 1998; Smith \& Sansom, 1999; Allen et al. 2000). However, this procedure is incorrect because it ignores the systematic influence of free-energy barriers and wells on the local movements of a diffusing particle (Roux \& Karplus, 1991a; Im \& Roux, 2002b). For example, an ion may appear to diffuse slowly in a region of the pore, while in fact the MSD is small because it is trapped in a free-energy well. To avoid double counting the effect of free-energy wells and barriers, it is important to separate the local dissipative forces from the systematic mean force. This problem can be addressed by considering an analogy with the overdamped random movements occurring during a $\mathrm{BD}$ trajectory of a particle along the axis $z$ in a potential $\mathcal{W}(z)$. We consider the dynamical displacement $\Delta_{z}$ of a particle that was at position $₹(t)$ at time $t$ and is found at $z(t+\Delta t)$ at a time $\Delta t$ later during an unbiased MD trajectory. According to the BD algorithm, the displacement is calculated as $\Delta_{z} \approx\left\langle\Delta_{z}\right\rangle+R$, where $\left\langle\Delta_{z}\right\rangle=D \mathcal{F} \Delta t / k_{\mathrm{B}} T$ is the systematic displacement of the particle under the influence of the mean force $\mathcal{F}$, and $R$ is a Gaussian random number with zero mean and standard deviation $\left\langle R^{2}\right\rangle=2 D \Delta t$. If follows that the diffusion coefficient can be estimated from the average fluctuations of the random dynamical steps

$D(z)=\frac{\left\langle\left[\Delta_{z}-\left\langle\Delta_{z}\right\rangle\right]^{2}\right\rangle_{z(t)}}{2 \Delta t}$

where the subscript implies that the diffusing ion was located at $z$ at time $t$. If the dynamical displacements corresponded to a purely Brownian motion, $\Delta t$ could be chosen arbitrarily small. In reality $\Delta t$ must be sufficiently large to incorporate all dynamical memory effects present in solution. According to the velocity autocorrelation functions of ions in solution (Lyubartsev \& Laaksnen, 1996) and estimated memory functions of ions inside channels (see Fig. 3), $\Delta t$ must be approximately $0 \cdot 5-1.0$ ps. In practice, the function $D(z)$ is extracted from unbiased MD trajectories by dividing the $z$-axis into intervals (bins) and averaging over a large number of dynamical steps which all started from $z$ in a given interval.

Dissipative factors can also be calculated from biased simulations. For example, when the reaction coordinate is Cartesian, the value of the diffusion can be obtained, via the Einstein relation $D=k_{\mathrm{B}} T / \gamma$, by calculating the friction coefficient, $\gamma$, from a MD trajectory during which the ion is fixed at some position along the $z$-axis (all other coordinates including the $x$ and $y$ coordinates of the ion are allowed to move). The friction coefficient is defined 
in terms of a time correlation function

$\gamma(z)=\frac{1}{k_{\mathrm{B}} T} \int_{0}^{\infty} \mathrm{d} t\left\langle\delta F_{z}(t) \delta F_{z}(0)\right\rangle_{(z)}$,

where $\delta F_{z}(t)=F_{z}(t)-\left\langle F_{z}\right\rangle_{(\vartheta)}$, is the deviation of the instantaneous force relative to the average force acting in the ₹ direction on the constrained ion (Berne et al. 1988, 1990; Roux \& Karplus, 1991a). Lastly, it is also advantageous to design a method to extract the diffusion coefficient from the biased simulations generated during umbrella sampling. This can be done using a general technique proposed by Straub and Berne (Berne et al. 1988; Straub et al. 1990). The technique is based on an analysis of the velocity autocorrelation function in terms of a GLE,

$\frac{k_{\mathrm{B}} T}{\left\langle\dot{z}^{2}\right\rangle_{(i)}} \dot{C}\left(t ; z_{i}\right)=-\frac{k_{\mathrm{B}} T}{\left\langle\delta z^{2}\right\rangle_{(i)}} \int_{0}^{t} \mathrm{~d} t^{\prime} C\left(t^{\prime} ; z_{i}\right)-\int_{0}^{t} \mathrm{~d} t^{\prime} M\left(t-t^{\prime} ; z_{i}\right) C\left(t^{\prime} ; z_{i}\right)$,

where and $C\left(t ; z_{i}\right)$ is the $z$-dependent velocity autocorrelation function $\langle\dot{z}(t) \dot{z}(0)\rangle_{(i)}$, and $\delta_{z}$ represents the deviation of $z$ from its average, $\delta_{z}=z-\langle z\rangle_{(i)}$. The subscript $i$ indicates that all averages in Eq. (110) are performed in the presence of a biasing harmonic window potential $u_{i}$. The diffusion coefficient at $z_{i}$ is related to the $s \rightarrow 0$ limit of the Laplace transform of the memory function,

$\hat{M}\left(s ; z_{i}\right)=\int_{0}^{\infty} \mathrm{d} t \mathrm{e}^{-s t} M\left(t ; z_{i}\right)$,

through Einstein's relation

$D\left(z_{i}\right)=\lim _{s \rightarrow 0} \frac{k_{\mathrm{B}} T}{\hat{M}\left(s ; z_{i}\right)}$.

The latter yields, after some algebra (Crouzy et al. 1994; Woolf \& Roux, 1994a),

$D\left(z_{i}\right)=\lim _{s \rightarrow 0} \frac{-\hat{C}\left(s ; z_{i}\right)\left\langle\delta z^{2}\right\rangle_{(i)}\left\langle\dot{z}^{2}\right\rangle_{(i)}}{\hat{C}\left(s ; z_{i}\right)\left[s\left\langle\delta z^{2}\right\rangle_{(i)}+\left\langle\dot{z}^{2}\right\rangle_{(i)} / s\right]-\left\langle\delta z^{2}\right\rangle_{(i)}\left\langle\dot{z}^{2}\right\rangle_{(i)}}$.

In practice, it is necessary to extrapolate from small values of $s$ to avoid the numerical instabilities as $s \rightarrow 0$.

\section{I.4 About computational times}

It is of interest to compare the computer time required by the various computational approaches for widely different channels. We assume that the concentration of permeant ions is sufficiently high to saturate the pore (e.g. 1-2 M KCl). Under those conditions, the conductance should be very close to the ideal maximum conductance $g_{\max }$. The maximum conductance is $\sim 30 \mathrm{pS}$ for the gA channel (Andersen \& Koeppe, 1992), $500 \mathrm{pS}$ for the KcsA channel (LeMasurier et al. 2001; Bernèche \& Roux, 2003), and 1250 pS for OmpF (Im \& Roux, 2002a) respectively. The time needed to observe 10 complete ion-crossing events during an equilibrium MD simulation, estimated using Eq. (102), is $\sim 1 \mu$ s for gA, $62 \mathrm{~ns}$ for KcsA, and $25 \mathrm{~ns}$ for OmpF. Assuming ohmic behavior, the time required to observe 10 ion crossing becomes considerably smaller if a membrane potential of $150 \mathrm{mV}$ is applied, being 356 ns for $\mathrm{gA}, 21 \mathrm{~ns}$ for KcsA, and $8 \mathrm{~ns}$ for $\mathrm{OmpF}$. In comparison, the computational requirements are very different if the 
permeation process is characterized by performing biased MD simulations to calculate a PMF. In a recent calculation of the 1 ion PMF for $\mathrm{K}^{+}$going through $\mathrm{gA}, \mathrm{y} \sim 100$ umbrella sampling windows of $1 \mathrm{~ns}$ each were generated, for a total of $100 \mathrm{~ns}$ (Allen et al. 2003b). However, the PMF converged in less than half of this time. In the case of KcsA, a 3-ion PMF was calculated using 340 umbrella-sampling windows of 100 ps, for a total of 34 ns (Bernèche \& Roux, 2001).

Evaluating the gain in computational efficiency with PMF calculations can be, however, quite subjective. For example, the computational time gained by using umbrella-sampling PMF simulations may become arbitrarily large compared to straight unbiased MD if there is a large free-energy barrier. Interestingly, even when there is no large free-energy barrier, it is still computationally more efficient to generate a number of biased simulations than to generate long unbiased simulations (van Duijneveldt \& Frenkel, 1992; Roux, 1995). This can be illustrated using a simple example. Assuming that the dynamics of the umbrella-sampling coordinate is governed by a simple friction coefficient $\gamma$, the sampling of the window histogram (in one dimension) should take place on a time-scale of $\tau_{\mathrm{w}} \sim \gamma / K$, where $K$ is the force constant of the harmonic window potential. If $N_{\mathrm{w}}$ simulations are used to cover the whole range $L$, the force constant $K$ of the umbrella-sampling potential must be chosen to ensure a proper overlap between the adjacent windows, i.e. each window should cover a range of $\Delta L=L / N_{\mathrm{w}}$ and the value of $K$ should be in the order of $k_{\mathrm{B}} T / \Delta L^{2}$, based on the magnitude of the RMS fluctuations. It follows that the total simulation time $T_{\text {tot }}$ needed to generate the $N_{\mathrm{w}}$ windows varies as $\sim L^{2} / N_{\mathrm{w}}$, decreasing with the number of windows. One further advantage of PMF calculations is that the individual biased simulations in umbrella sampling do not need to communicate from one another and can be generated independently. This leads to the possibility of performing 'coarse-grained' calculations that are massively distributed over a large number of relatively inexpensive computers. Therefore, the PMF calculations appear to be efficient tools to characterize ion permeation.

\section{2 lon permeation models}

\subsection{The ID-NP electrodiffusion theory}

Assuming that we now have calculated the PMF and the diffusion coefficient profile from MD, how can we use these quantities to quantitatively assess the ion permeation of a channel? The simplest situation corresponds to that of a channel that can, at most, contain a single permaent ion at a time. The motion of the ion could easily be represented as random trajectories using BD (Cooper et al. 1985; Jakobsson \& Chiu, 1987). However, in this simple case, the diffusion process can be treated analytically by using the 1D-NP equation to represent the net stationary flux $J$ of ions through the channel (Levitt, 1986)

$J=-D(z) \frac{\mathrm{d} P(z)}{\mathrm{d} z}-P(z) \frac{D(z)}{k_{\mathrm{B}} T} \frac{\mathrm{d} \mathcal{W}(z)}{\mathrm{d} z}$,

where $P(z)$ is the probability density per unit length of finding the ion, $D(z)$ is the ion diffusion coefficient and $\mathcal{W}(z)$ is the total free-energy profile of the ion along the channel axis. According to Eq. (89), the total free-energy profile can be expressed as a sum of a first contribution corresponding to the equilibrium PMF, and a second contribution arising from the presence of the electrostatic potential across the membrane (see Section 4.3). To construct suitable '1-ion pore' boundary conditions for the 1D-NP equation, it is assumed that the ends of the pore, 
at $\pm L / 2$, are in equilibrium with the solution with which they are in contact (Levitt, 1986). The linear probability density $P(z)$ is related to the bulk concentrations $c_{1}$ and $c_{2}$ by

$$
\begin{aligned}
P(-L / 2) & =c_{1} P_{0} \int \mathrm{d} t^{\prime} \delta\left(z^{\prime}-L / 2\right) \mathrm{e}^{-\mathcal{W}_{3 \mathrm{D}}\left(\mathbf{r}^{\prime}\right) / k_{\mathrm{B}} T} \\
& =c_{1} P_{0} S \mathrm{e}^{-\mathcal{W}_{1 \mathrm{D}}(z=-L / 2) / k_{\mathrm{B}} T},
\end{aligned}
$$

where S corresponds to an effective cross-section defined by Eq. (98). A similar equation holds for $P(+L / 2)$. The factor $P_{0}$ in Eq. (115) is the total probability that the channel is empty

$P_{0}=1-\int_{-L / 2}^{+L / 2} \mathrm{~d} z^{\prime} P\left(z^{\prime}\right)$.

This empirically enforces the constraint that no more than a single ion can simultaneously occupy the channel. If the concentration of ions is increased, the occupation of the pore will saturate to one ion per channel according to Eq. (78). The boundary conditions Eq. (115) could be (or must be) modified to account for the access resistance at the pore's entrance and for interfacial polarization (Andersen, 1983; Chiu \& Jakobsson, 1989). The 1D-NP model can be solved analytically in closed form, for any concentration and transmembrane potential (Levitt, 1986). For a small transmembrane potential $V_{\mathrm{mp}}$, the net current is expected to obey a linear relationship following Ohm's law, $I=g(c) V_{\mathrm{mp}}$, where $g$ is the concentration-dependent conductance of the channel. Under such conditions, the channel conductance follows a simple firstorder saturation relation

$g(c)=\frac{K_{1} c}{1+K_{1} c} g_{\max }$,

where $K_{1}$ is the 1 -ion equilibrium-binding constant defined by Eq. (99). The maximum conductance $g_{\max }$ is,

$g_{\max }=\frac{D_{\mathrm{eff}}}{L^{2}} \frac{q^{2}}{k_{\mathrm{B}} T}$,

where $D_{\text {eff }}$ is an effective diffusion coefficient of the ion in the pore

$D_{\text {eff }}=\left\langle D(z)^{-1} \mathrm{e}^{+\mathcal{W}_{\mathrm{eq}}(\mathrm{z}) / k_{\mathrm{B}} T}\right\rangle^{-1}\left\langle\mathrm{e}^{-\mathcal{W}_{\mathrm{eq}}(\mathrm{z}) / k_{\mathrm{B}} T}\right\rangle^{-1}$,

calculated as a spatial average over the full length of the pore, $-L / 2<z<+L / 2$.

\subsubsection{Discrete-state Markov chains}

We saw above that the 1D-NP equation provides a very convenient framework for describing ion permeation through a channel using the PMF and diffusion coefficient profile calculated from MD for a single-ion pore. However, it is generally not possible to solve the diffusion problem analytically in closed form in the case of a multi-ion pore. In this case, it is more practical to extract the non-equilibrium properties of the system using $\mathrm{BD}$ trajectories. One possible treatment is to map the problem onto a continuous-time Markov chain with discrete states corresponding to the ion positions, and generate the trajectory of the ions as a stateto-state random walk using exponentially distributed random survival times. Such a treatment is computationally efficient and numerically stable. This feature is particularly attractive when 
a PMF calculated from MD and stored on a discrete grid is being used. The forward and backward transition rates are given by (Agmon \& Hopfield, 1983; Schumaker et al. 2000;' Bernèche \& Roux, 2003)

$k_{\left[\left(\ldots z_{i}, \ldots\right) \rightarrow\left(\ldots z_{i} \pm \delta \delta_{z}, \ldots\right)\right]}=\left(\frac{D\left(z_{i}\right)+D\left(z_{i} \pm \delta_{z}\right)}{2 \delta z^{2}}\right) \mathrm{e}^{-\left[\mathcal{W}\left(\ldots, z_{i} \pm \delta \gamma_{z} \ldots\right)-\mathcal{W}\left(\ldots, z_{i}, \ldots\right)\right] / 2 k_{\mathrm{B}} T}$,

where $D(z)$ is the space-dependent diffusion coefficient of the ions in the channel, and $\delta_{z}$ is the grid spacing. The random walk generated by Eq. (120) satisfies the condition of detailed balance under equilibrium conditions in the absence of net flux, and the stochastic evolution of the system obeys the multi-dimensional Smoluchowski diffusion Eq. (10) as $\delta_{z}$ becomes increasingly small (McGill \& Schumaker, 1996; Schumaker et al. 2000, 2001). The resulting equations are closely related to the coupled multi-ion diffusion theory that was formulated by Stephan et al. (1983). However, it should emphasized that such framework model differs essentially from traditional kinetic models based on classical Eyring Rate Theory (ERT) (Hille, 2001). Entry and exit of ions into the simulation region can be represented as a first-order process. In the case of a channel that can be occupied by $n$ ions, one ion can attempt to exit the system as soon as it reaches the ends of the pore, at $z_{\min }$ or at $z_{\max }$. The exit rate of the ion at $z_{\min }$ is

$k_{\text {exit }}=\left(\frac{D\left(z_{\text {min }}\right)}{2 \delta z^{2}}\right) \mathrm{e}^{-\left[\mathcal{W}\left(z_{1}, z_{2}, \ldots, z_{i-1}\right)-\mathcal{W}\left(z_{1}, z_{2}, \ldots, z_{i}=z_{\text {min }}\right)\right] / 2 k_{\mathrm{B}} T}$.

An ion can attempt to enter a two-ion occupied channel at any time with a rate (e.g. on the intracellular side)

$k_{\text {entry }}=c_{\text {int }} S \delta_{z}\left(\frac{D\left(z_{\text {min }}\right)+D_{\text {bulk }}}{2 \delta_{z^{2}}}\right) \mathrm{e}^{-\left[\mathcal{W}\left(z_{1}, z_{2}, \ldots, z_{i-1}, z_{i}=z_{\min }\right)-\mathcal{W}\left(z_{1}, z_{2}, \ldots, z_{j-1}\right)\right] / 2 k_{\mathrm{B}} T}$,

where $c_{\text {int }}$ is the ion concentration on the intracellular side, and $\mathrm{S}$ is the cross-sectional area of the vestibule. Similar expression holds for the entry and exit on the extracellular side. Because it is so generic, the continuous-time Markov chain framework model is related to other approaches such as the dynamic lattice Monte Carlo model of Graf et al. (2000), where the ions are undergoing a random walk on a discrete grid. One important difference is that the theory of Graf et al. (2000) describes all interactions on the basis of continuum electrostatics whereas a PMF and a diffusion coefficient profile calculated from all-atom MD are used as inputs in the framework model described by Eqs. (120)-(122).

\subsubsection{The GCMC/BD algorithm}

In the case of wide aqueous pores, a large number of ions and counterions may be required to describe the permeation process. Calculating all the multi-ion PMFs using all-atom MD simulations may not be feasible. In such a case, assuming that water is a dielectric continuum may be a valid and useful approximation to calculate the multi-ion PMFs. Furthermore, the total number of ions must be allowed to fluctuate under the influence of the specific non-equilibrium boundary conditions because ions can enter and leave such an open system. This can be accomplished by combining the BD stochastic dynamics with the GCMC algorithm (Im et al. 2000).

The implementation of the GCMC/BD algorithm (Im et al. 2000) for simulating the diffusion of ions through a channel embedded in a bilayer membrane surrounded by aqueous salt solutions 


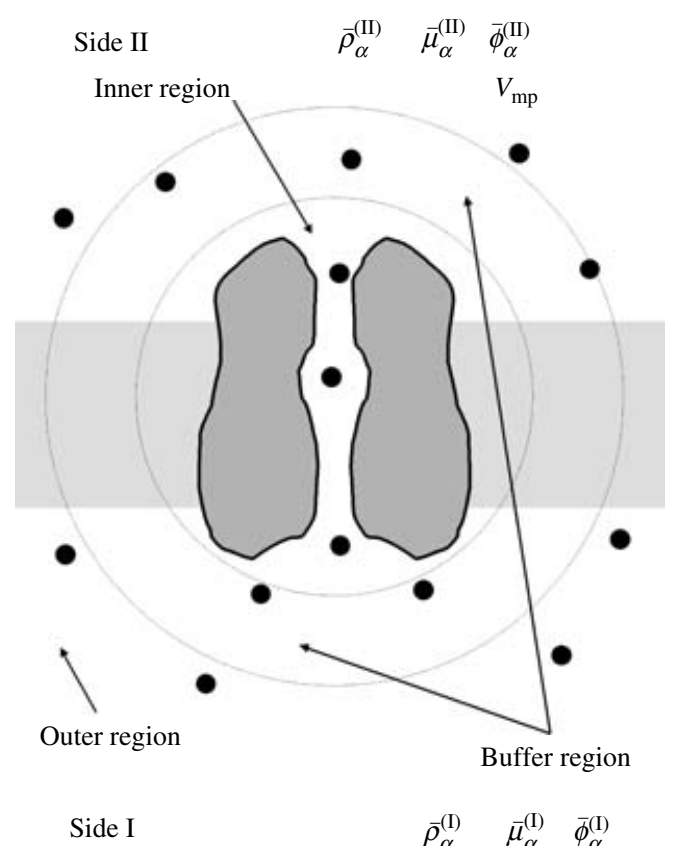

Fig. 9. Schematic representation of the Grand Canonical Monte Carlo Brownian dynamics (GCMC/BD) algorithm. As in Fig. 8, the transmembrane potential is 0 on side $\mathrm{I}$ and $V_{\mathrm{mp}}$ on side II. The ions in the inner and buffer regions evolve according to Eq. (123) with the total multi-ion PMF given in Eq. (124). In the buffer regions, ions can be created with probability Eq. (129) and destroyed with probability Eq. (130) according to the GCMC algorithm.

is schematically illustrated in Fig. 9. A spherical geometry for the simulation region is shown for convenience, though other choices are also possible. The system is divided into five specific spatial regions: the inner region, the buffer regions on sides I and II, and the outer regions on sides I and II. There are $n$ explicit ions in the 'simulation region' (inner and buffer regions); all other ions in the bulk are excluded from the pore region and are restricted to the bulk region where they are represented implicitly. The channel is assumed to be rigidly fixed in space and water is represented as a continuum dielectric in all the regions. The $\mathrm{BD}$ trajectory of the $n$ ions is generated according to the algorithm of Ermark (1975),

$\mathbf{r}_{i}(t+\Delta t)=\mathbf{r}_{i}(t)+\left[\frac{D_{i}}{k_{\mathrm{B}} T} \mathcal{F}_{i}+\nabla_{i} D_{i}\right] \Delta t+\boldsymbol{\zeta}_{i}$

where $\Delta t$ is the BD timestep, $D_{i}\left(\mathbf{r}_{i}\right)$ is the diffusion coefficient of the ion, and $\zeta_{i}$ is a random Gaussian vector with zero average and $\left\langle\xi_{i} \cdot \zeta_{i}\right\rangle=6 D_{i} \Delta t$. The forces acting on the ions are calculated from the gradient of the multi-ion PMF $\mathcal{W}$ of the system according to Eq. (4). The total multi-ion PMF is written as (Im \& Roux, 2001)

$\mathcal{W}\left(\mathbf{r}_{1}, \mathbf{r}_{2}, \ldots\right)=\sum_{i<j} \mathcal{W}_{\text {bulk }}^{(i j)}\left(\left|\mathbf{r}_{i}-\mathbf{r}_{j}\right|\right)+\sum_{i} U_{\text {core }}\left(\mathbf{r}_{i}\right)+\sum_{i} q_{i} \phi_{\mathrm{sf}}\left(\mathbf{r}_{i}\right)+\Delta \mathcal{W}_{\mathrm{rf}}\left(\mathbf{r}_{1}, \mathbf{r}_{2}, \ldots\right)$,

where $\mathcal{W}_{\text {bulk }}^{(i j)}$ is the spherically symmetric PMF between ions $i$ and $j$ in an isotropic bulk solution (Pettitt \& Rossky, 1986; Guàrdia \& Pardró, 1991; Llano-Restrepo \& Chapman, 1994), 
$U_{\text {core }}$ is the non-electrostatic repulsive core overlap potential (excluding the ions from the interior of the protein or the membrane), $\phi_{\mathrm{sf}}$ is the static field arising from the channel charges and the transmembrane potential, and $\Delta \mathcal{W}_{\text {rf }}$ is the electrostatic reaction-field free energy of the ions in the system. The ion-ion PMF $\Delta \mathcal{W}_{\text {bulk, }}^{i j}$ which incorporates the short-range hydration structure, is expressed in Eq. (26) (Moy et al. 2000; Im \& Roux, 2002a). The static field is calculated using the PB-V equation formulated in Section 3.6

$\boldsymbol{\nabla} \cdot\left[\varepsilon(\mathbf{r}) \boldsymbol{\nabla} \phi_{\mathrm{sf}}(\mathbf{r})\right]-H_{\mathrm{bulk}}(\mathbf{r}) \bar{\kappa}^{2}(\mathbf{r})\left[\phi_{\mathrm{sf}}(\mathbf{r})-V_{\mathrm{mp}} \Theta(\mathbf{r})\right]=-4 \pi \rho_{\mathrm{c}}(\mathbf{r})$,

where $\rho_{\mathrm{c}}(\mathbf{r})$ is the charge density of the channel. On the basis of the developments of Section 4.3, the function $H_{\text {bulk }}(\mathbf{r})$ is set to 1 in the outer (bulk) regions (where the ions are represented implicitly) and set to zero in the inner and buffer regions (where the ions are represented explicitly). [See also Eq. (82).] The electrostatic fields arising from the protein charges and the potential difference across the membrane could be calculated separately by setting $V_{\mathrm{mp}}=0$ or $\rho_{\mathrm{c}}(\mathbf{r})=0$ in Eq. (125), respectively. By virtue of the linearity of Eq. (125), they are simply superimposed in $\phi_{\mathrm{sf}}(\mathbf{r})$. The contribution of the reaction field to the multi-ion PMF can be expressed as (Im \& Roux, 2001)

$$
\begin{aligned}
\Delta \mathcal{W}_{\mathrm{rf}}\left(\mathbf{r}_{1}, \mathbf{r}_{2}, \ldots\right) & =\frac{1}{2} \int \mathrm{dr} \mathrm{d} \mathbf{r}^{\prime} \rho_{\text {ions }}(\mathbf{r}) G_{\mathrm{rf}}\left(\mathbf{r}, \mathbf{r}^{\prime}\right) \rho_{\text {ions }}\left(\mathbf{r}^{\prime}\right) \\
& =\frac{1}{2} \sum_{i} q_{i} \phi_{\mathrm{rf}}\left(\mathbf{r}_{i}\right),
\end{aligned}
$$

where $\rho_{\text {ions }}(\mathbf{r})$ is the charge density of all the explicit ions in the system and $G_{\mathrm{rf}}(\mathbf{r}, \mathbf{r})$ is Green's function corresponding to the reaction-field potential at $\mathbf{r}$ arising from a point charge located at $\mathbf{r}^{\prime}$. It may be noted that $G_{\mathrm{rf}}\left(\mathbf{r}, \mathbf{r}^{\prime}\right)$ depends crucially on the shape of all the dielectric boundaries in the system. The reaction field is calculated as $\phi_{\mathrm{rf}}(\mathbf{r})=\left[\phi_{\text {syst }}(\mathbf{r})-\phi_{\text {bulk }}(\mathbf{r})\right]$, where $\phi_{\text {syst }}(\mathbf{r})$ is the electrostatic potential computed in the full system, with the complex solvent-channel dielectric boundaries,

$\boldsymbol{\nabla} \cdot\left[\varepsilon(\mathbf{r}) \nabla \phi_{\text {syst }}(\mathbf{r})\right]-H_{\text {bulk }}(\mathbf{r}) \bar{\kappa}^{2}(\mathbf{r}) \phi_{\text {syst }}(\mathbf{r})=-4 \pi \rho_{\text {ions }}(\mathbf{r})$

and $\phi_{\text {bulk }}(\mathbf{r})$ is the electrostatic potential computed for a reference system corresponding to a uniform bulk solvent at zero salt concentration.

$\varepsilon_{\mathrm{w}} \boldsymbol{\nabla}^{2} \phi_{\text {bulk }}(\mathbf{r})=-4 \pi \rho_{\text {ions }}(\mathbf{r})$.

In principle, $G_{\mathrm{rf}}\left(\mathbf{r}, \mathbf{r}^{\prime}\right)$ could be calculated once and stored, but it is a function of six independent cartesian coordinates and this can require substantial memory resources and computations for channels of arbitrary geometries. These difficulties are circumvented by expressing the ion charge distribution in the simulation region using a set of normalized basis functions (Im \& Roux, 2001). Lastly, it should be noted that, while the decomposition described by Eq. (124) is not unique, each separate contribution can be determined without ambiguity.

To ensure that proper external conditions are maintained on the boundaries of the inner region, the ions in buffer regions I and II on both sides of the membrane are kept in equilibrium with the bulk solution with which they are in contact. This is enforced via the GCMC algorithm with particle creations and destructions in the two buffer regions (Im et al. 2000). Given that the system contains $n_{\alpha}$ ions of type $\alpha$, the creation probability of an ion of that type in a randomly 
selected buffer region is

$P_{\text {creat }}=\frac{\left.\left(\bar{n}_{\alpha} /\left(n_{\alpha}+1\right)\right) \exp \left[-\Delta \mathcal{W}-\bar{\mu}_{\alpha}\right) / k_{\mathrm{B}} T\right]}{1+\left(\bar{n}_{\alpha} /\left(n_{\alpha}+1\right)\right) \exp \left[-\left(\Delta \mathcal{W}-\bar{\mu}_{\alpha}\right) / k_{\mathrm{B}} T\right.}$,

where $\bar{n}_{\alpha}$ is the expectancy for the number of ions of type $\alpha$ from the bulk density $\bar{\rho}_{\alpha}$ and the volume of the buffer region, $\bar{\mu}_{\alpha}$ is the chemical potential, and $\Delta \mathcal{W} \equiv\left[\mathcal{W}\left(\ldots, n_{\alpha}+1, \ldots\right)\right.$ $\left.-\mathcal{W}\left(\ldots, n_{\alpha}, \ldots\right)\right]$ is the change in the total multi-ion PMF of the system due to the new ion. The destruction probability of an ion of type $\alpha$ is

$P_{\text {destr }}=\frac{1}{1+\left(\bar{n}_{\alpha} / n_{\alpha}\right) \exp \left[-\left(\Delta \mathcal{W}-\bar{\mu}_{\alpha}\right) / k_{\mathrm{B}} T\right]}$,

where $\Delta \mathcal{W} \equiv\left[\mathcal{W}\left(\ldots, n_{\alpha}, \ldots\right)-\mathcal{W}\left(\ldots, n_{\alpha}-1, \ldots\right)\right]$ is the change in the total multi-ion PMF of the system due to particle removal. The chemical potential of ions of type $\alpha$ in each buffer region is specified by

$$
\left.\begin{array}{rl}
\bar{\mu}_{\alpha}(\mathrm{I})=\Delta \mu_{\alpha}(\mathrm{I}) & \text { side I } \\
\bar{\mu}_{\alpha}(\mathrm{II})=\Delta \mu_{\alpha}(\mathrm{II})+q_{\alpha} V_{\mathrm{mp}} & \text { side II }
\end{array}\right\}
$$

where $V_{\mathrm{mp}}$ is the imposed transmembrane potential, and $\Delta \mu_{\alpha}(\mathrm{I})$ and $\Delta \mu_{\alpha}$ (II) are the excess chemical potential in bulk solution. The latter are influenced by ion-ion interactions in the bulk solution and are, thus, concentration-dependent [see Eq. (36)]. The multi-ion PMF takes into account the interactions between all the ions present in the simulation region and the influence of the channel, the transmembrane potential as well as implicit salt in the outer region.

The GCMC procedure has the effect of enforcing boundary conditions corresponding to constant electro-chemical potential in the two buffer regions and can be used to simulate equilibrium as well as non-equilibrium conditions of ion diffusion and permeation (Im et al. 2000). One cycle of GCMC/BD corresponds to one step of BD followed by a few steps of GCMC (typically 1-10) to maintain the buffer regions in equilibrium. No ion creation or destruction are taking place in the inner region and the time-course of the ions in the inner system evolves dynamically according to $\mathrm{BD}$. When the system is at equilibrium, the electrochemical potential of any ion is the same in all the regions of the system and there is no net flow. However, when non-equilibrium conditions are imposed at the boundaries, a stationary state is simulated as particles flow from the regions with a high value of electro-chemical potential to the regions with lower values. Since the buffer regions cannot run out of particles nor be filled by particles, they essentially act as infinite thermodynamic reservoirs and sinks for the particles with respect to the central inner region. The GCMC/BD algorithm (Im et al. 2000) is closely related to the dual-volume-control molecular dynamics method (DCV/MD) (Heffelfinger \& Ford, 1998; Thompson et al. 1998; Pohl \& Heffelfinger, 1999; Thompson \& Heffelfinger, 1999) that has been used to simulate the diffusion of gases across porous membranes.

The GCMC procedure described above does not present a significant additional burden to standard BD simulations. This can be understood using simple arguments. The evaluation of the forces acting on the ions is required at every BD time-step of the stochastic trajectory. Assuming that $n$ ions are present, the calculation of the ion-ion forces is of order $n(n-1) / 2$. In contrast, each GCMC trial requires only energy differences caused by the insertion or deletion of a single ion and are of order $n$. Furthermore, by choosing the position and dimension of 
the buffer region wisely, it is possible to reduce the size of the system and focus on the most important parts of a channel and thus further decrease the computational expense.

\subsubsection{PNP electrodiffusion theory}

PNP continuum electrodiffusion theory represents the average ion fluxes in terms of densities and potential gradients (Kurnikova et al. 1999; Cardenas et al. 2000; Hollerbach et al. 2000, 2002; Hille, 2001; Schuss et al. 2001; Gillespie et al. 2002; Im \& Roux, 2002a; Koumanov et al. 2003; Mamonov et al. 2003).

$\mathbf{J}_{\alpha}(\mathbf{r})=-D_{\alpha}(\mathbf{r})\left(\boldsymbol{\nabla} \rho_{\alpha}(\mathbf{r})+\frac{\rho_{\alpha}(\mathbf{r})}{k_{\mathrm{B}} T} \nabla \mathcal{W}_{\alpha}^{\text {eff }}(\mathbf{r})\right)$,

where $D_{\alpha}(\mathbf{r})$ is the diffusion coefficient, $\rho_{\alpha}(\mathbf{r})$ is the density, and $\mathcal{W}_{\alpha}^{\text {eff }}(\mathbf{r})$ is an effective potential acting on the ions. By continuity, the average density of the ion of type $\alpha$ also obeys

$\frac{\partial \rho_{\alpha}(\mathbf{r}, \mathrm{t})}{\partial t}+\boldsymbol{\nabla} \cdot \mathbf{J}_{\alpha}(\mathbf{r})=0$

Under steady-state conditions, the densities do not vary with time and $\nabla \cdot \mathbf{J}_{\alpha}=0$. The effective potential is represented as

$\mathcal{W}_{\alpha}^{\text {eff }}(\mathbf{r})=U_{\text {core }}(\mathbf{r})+q_{\alpha} \phi(\mathbf{r})$,

where $U_{\text {core }}(\mathbf{r})$ is the non-electrostatic core repulsive potential (excluding the ions from the interior of the protein or the membrane) and $\phi(\mathbf{r})$ is the average electrostatic potential arising from all the interactions in the system, which is calculated from the Poisson equation (Jackson, 1962).

$\boldsymbol{\nabla} \cdot[\varepsilon(\mathbf{r}) \nabla \phi(\mathbf{r})]=-4 \pi\left(\rho_{\mathrm{c}}(\mathbf{r})+\sum_{\alpha} q_{\alpha} \rho_{\alpha}(\mathbf{r})\right)$.

Because the NP Eq. (132) and the Poisson Eq. (135) are coupled partial differential equations, they must be solved in a self-consistent manner for the electrostatic potential $\phi(\mathbf{r})$, and ion concentrations $\rho_{\alpha}(\mathbf{r})$. For this reason, PNP is called a 'mean-field theory' (see also Section 2.3). The early form of such theory can be traced back to Nernst (1890) and Onsager $(1926,1927)$. Equation (132) is equivalent to a 1-ion Smulochowski diffusion equation (Smoluchowski, 1916; Chandrasekar, 1943) driven by the effective potential $\mathcal{W}_{a}^{\text {eff }}(\mathbf{r})$. PNP is a mean-field theory which treats discrete ions in channel as a continuum charge distribution. In the absence of any net flux [i.e. $\mathbf{J}_{\alpha}(\mathbf{r})=0$ for all $\alpha$ ], the PNP theory becomes equivalent to the equilibrium nonlinear PB continuum Eq. (29). As highlighted by Moy et al. (2000) and Corry et al. (2000), this can lead to serious problems and the theory must obviously be used with caution. Ultimately, the meanfield construction underlies all non-equilibrium and equilibrium properties of the theory.

The PNP theory has been applied to simple cylindrical pores (Corry et al. 2000; Koumanov et al. 2003), the gA channel (Kurnikova et al. 1999; Cardenas et al. 2000; Hollerbach et al. 2000, 2002; Mamonov et al. 2003) and OmpF porin (Im \& Roux, 2002a). Kurnikova et al. (1999) proposed a robust and stable algorithm allowing numerical solution of the PNP equations in 3D space to solve Eqs. (132) and (135). To solve coupled PNP Eqs. (132) and (135) according 
to this algorithm, the flux density $\mathbf{J}_{\alpha}(\mathbf{r})$ in Eq. (132) is re-written as

$\mathbf{J}_{\alpha}(\mathbf{r})=-D_{\alpha}(\mathbf{r}) \mathrm{e}^{-\mathcal{W}_{\alpha}^{\text {eff }}(\mathbf{r}) / k_{\mathrm{B}} T} \boldsymbol{\nabla}\left[\rho_{\alpha}(\mathbf{r}) \mathrm{e}^{\mathcal{W}_{\alpha}^{\text {eff }}(\mathbf{r}) / k_{\mathrm{B}} T}\right]$.

Eq. (136) is then transformed to the Laplace equation assuming that the system is at a steady state,

$\boldsymbol{\nabla} \cdot\left[\tilde{D}_{\alpha}(\mathbf{r}) \boldsymbol{\nabla} \tilde{\rho}_{\alpha}(\mathbf{r})\right]=0$,

where $\tilde{D}_{\alpha}(\mathbf{r})=D_{\alpha}(\mathbf{r}) \mathrm{e}^{-\mathcal{W}_{\alpha}^{\text {eff }}(\mathbf{r}) / k_{\mathrm{B}} T}$ and $\tilde{\rho}_{\alpha}(\mathbf{r})=\rho_{\alpha}(\mathbf{r}) \mathrm{e}^{\mathcal{W}_{\alpha}^{\text {eff }}(\mathbf{r}) / k_{\mathrm{B}} T}$. Equation (137) is isomorphic to the Poisson Eq. (135). For this reason, both equations can be solved numerically in a selfconsistent manner on a discrete 3D grid using similar finite-difference relaxation method (Warwicker \& Watson, 1982b; Klapper et al. 1986). It is usually assumed that the non-electrostatic potential $U_{\text {core }}(\mathbf{r})$ in Eq. (132) corresponds to a hard repulsive potential by the channel and membrane which prevents the ions from entering forbidden regions. In practice, instead of handling $U_{\text {core }}(\mathbf{r})$ explicitly, the repulsive potential is implemented by setting $\rho_{\alpha}(\mathbf{r})=0$ to zero in non ion-accessible regions and setting the normal component of $\mathbf{J}_{\alpha}(\mathbf{r})$ to zero at the surface of the forbidden regions (Kurnikova et al. 1999; Cardenas et al. 2000). One cycle of a PNP iterations consists of solving the Poisson Eq. (135) for electrostatic potential and then the steadystate NP Eq. (137) for concentration of each ion type. To ensure the numerical stability during PNP iterations, a relaxation method can be implemented by performing a mixing of potential and concentrations in each step of iterations with the values in the previous iteration (Im \& Roux, 2002a). The Debye-Hückel approximation can be used to setup the boundary potential on the edge of the grid, for the Poisson Eq. (135) (Im \& Roux, 2002a). Because the potential obtained from $\mathrm{PB}$ is much closer to the correct answer than the unscreened potential from the Poisson equation without any background ion charge distribution, a reasonable initial guess of concentrations for the first PNP cycle is obtained by solving the linearized (or nonlinear) PB equation. Such a scheme can help significantly reduce both numerical instability and computational time. Once the equations have been solved self-consistently, the total current is the sum of the current carried by each ion species, $I=\sum_{\alpha} I_{\alpha}$.

\section{Computational studies of ion channels}

We have now reviewed and laid out the basic principles of a unified theoretical framework as well as the most important techniques for describing ion permeation. In this section, we will attempt to illustrate those concepts by reviewing the main results obtained in recent theoretical studies of three channels which have important similarities and differences: gA, KcsA and OmpF. They are illustrated in Figs 1 and 2. Let us first review the main features of these three channels.

The gA channel is currently one of the best characterized molecular pore, structurally and functionally (Andersen \& Koeppe, 1992; Roux \& Karplus, 1994; Hille, 2001). In a phospholipid bilayer membrane, this small pentadecapeptide adopts a head-to-head $\beta$-helical dimer conformation to form an ion-conducting narrow pore of $\sim 4 \AA$ in diameter (Arseniev et al. 1985; Ketchem et al. 1993, 1997; Townsley et al. 2001; Allen et al. 2003a). The channel is illustrated in Fig. 1a. The hydrogen-bonded carbonyls line the pore and the alternating $\mathrm{D}$ and L amino-acid side-chains, most of them hydrophobic, extend away into the membrane lipid. 
(a)

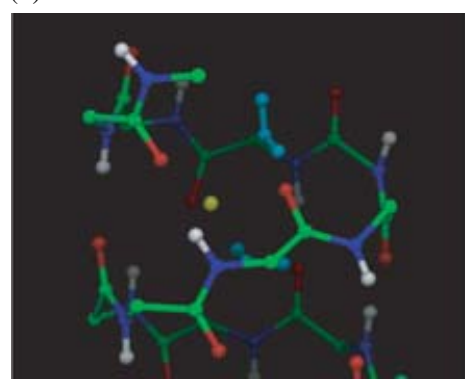

(b)

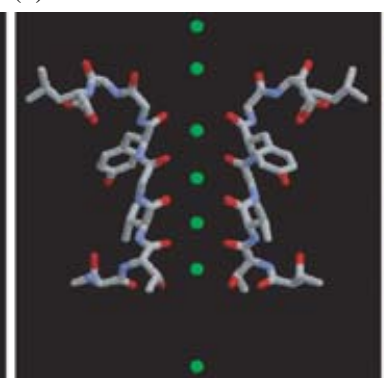

(c)

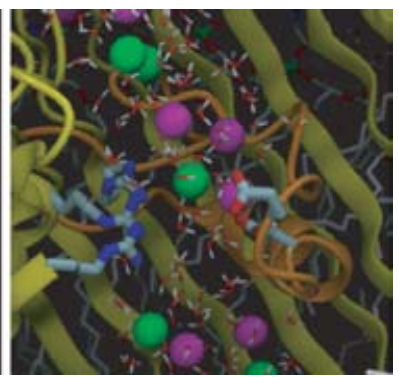

Fig. 10. (a) Ion in the inner binding site of the gramicidin A channel (Woolf \& Roux, 1997). (b) $\mathrm{K}^{+}$-binding sites in the selectivity filter of KcsA [(only two subunits are shown); from Zhou et al. 2001b]. (c) $\mathrm{K}^{+}$and $\mathrm{Cl}^{-}$ in the constriction zone of $\mathrm{OmpF}$.

The diameter of the pore is such that the permeation process involves the translocation in single file of the ion and water molecules through the channel interior, the large energetic loss due to dehydration being compensated by coordination with the backbone carbonyl oxygens (see Fig. 10a). The gA channel exhibits a functional behavior similar to far more complex macromolecular biological structures, and for this reason, has proved to be an extremely useful model system to study the principles governing ion transport across lipid membranes, both theoretically and experimentally (Andersen \& Koeppe, 1992).

The KcsA channel was the first biological potassium-selective channel for which a structure was determined at atomic resolution using X-ray crystallography (Doyle et al. 1998). The ion conducting pore is along the axis of symmetry of four identical membrane-spanning subunits. The channel is illustrated in Fig. 1b. The pore comprises a wide non-polar aqueous cavity on the intracellular side, leading up, on the extracellular side, to a narrow pore $12 \AA$ long, lined exclusively by main chain carbonyl oxygens. Formed by the residues corresponding to the signature sequence TTVGYG common to all $\mathrm{K}^{+}$channels, this region of the pore acts as a 'selectivity filter' by allowing only the passage of $\mathrm{K}^{+}$ions across the cell membrane, whereas the wide vestibular cavity helps overcome the desolvation barrier due to the cell membrane (Roux \& MacKinnon, 1999). The width of the selectivity filter is such that a permeating $\mathrm{K}^{+}$ion must shed most of its surrounding waters. The large energetic loss due to dehydration being compensated by coordination with the main chain carbonyl oxygens (see Fig. 10b). The movement $\mathrm{K}^{+}$ions through this region is the rate-limiting step in the conduction mechanism. The ability of $\mathrm{K}^{+}$channels to conduct $\mathrm{K}^{+}$ions at nearly the diffusion limit is traditionally described in terms of concerted mechanisms in which ion-channel attraction and ion-ion repulsion play compensating effects as several $\mathrm{K}^{+}$ions are moving simultaneously in single file through the narrow pore (Hodgkin \& Keynes, 1955; Hille \& Schwarz, 1978; Neyton \& Miller, 1988a, c).

Porins from the outer membrane of Eschericha coli are large macromolecular structures which allow the diffusion of hydrophilic molecules with a molecular weight up to $600 \mathrm{Da}$ and exhibit modest ionic selectivity. For a recent review see Schirmer (1998). The cation-selective matrixporin $(\mathrm{OmpF})$ is produced under normal conditions, while the anion-selective phsophoporin (PhoE) is expressed under phosphate limitations. OmpF was the first membrane protein crystallized with detergent (though the structure determination was completed almost a decade later). The channel is illustrated in Fig. $1 c$. OmpF and PhoE, as most of the porins, folds into similar homo-trimeric structures (Schirmer, 1998). Each monomer consists of a 16-stranded 
Table 1. Total potential of mean force $(P M F) \mathcal{W}=\mathcal{W}_{\mathrm{eq}}+\mathcal{W}_{\mathrm{mp}}$

\begin{tabular}{llll}
\hline \hline Acronym & Contribution & Microscopic systems & Equations \\
\hline PMF (MD) & $\mathcal{W}_{\text {eq }}$ & All region: all-atom MD with explicit solvent & $\begin{array}{c}(104) \text { or }(105) \\
\text { or }(106)\end{array}$ \\
Vmp (PB-V, MD) & $\mathcal{W}_{\mathrm{mp}}$ & $\begin{array}{c}\text { Pore region: all-atom MD with explicit solvent } \\
\text { Outer region: solvent continum }\end{array}$ & $(82)-(90)$ \\
Vmp (PB-V) & $\mathcal{W}_{\mathrm{mp}}$ & $\begin{array}{c}\text { All regions: atomic protein with solvent } \\
\text { and membrane dielectric continum }\end{array}$ & All regions: atomic protein with solvent and \\
PMF (PB-V) & $\mathcal{W}$ & membrane dielectric continum & $(124)-(128)$ \\
& & & \\
\hline \hline
\end{tabular}

$\beta$-barrel with eight short turns (T1-T8) at the periplasmic side and eight relatively large loops (L1-L8) at the cell surface which confers a significant stability and rigidity to the structure. Each monomer possesses a wide aqueous pore narrowed by the loops on the outer entrance of the $\beta$-barrel. One of these loops (L3) is folded inside the $\beta$-barrel forming a narrow region of $\sim 6 \AA$ diameter within the wide aqueous channel at roughly halfway through the membrane. This narrowest region of the pore, called the 'constriction zone', illustrated in Fig. 10c, is thought to be responsible for the charge specificity of the porins (Cowan et al. 1992).

As illustrated in Fig. 10, permeating ions going through these three channels experience widely different environments. Thus, comparing and contrasting the main features of the ion permeation mechanism for these three channels helps illustrate important fundamental principles. For example, many of the important principles governing ion permeation through narrow pores an be clarified by considering the gA and KcsA channels. In both cases, the large energy cost required for dehydrating the permeating ion must be compensated by strong and tight interactions with the protein channel. On the other hand, the importance of ion-ion interactions in multi-ion channels in the confined environment of a molecular pore may be examined by considering $\mathrm{Kcs}$ A and $\mathrm{OmpF}$. Furthermore, $\mathrm{OmpF}$ provides a great opportunity to examine the influence of counterions and electrostatic screening in an aqueous pore and assess the validity of approximate theories such as PB and PNP. Because they differ significantly, different computational framework may be more practical for the various systems. Those differences concern mostly the construction of the total PMF that governs ion permeation. The different models are listed in Table 1.

\section{I Computational Studies of gA}

Computer simulations of atomic models of the gA channel have a long history that dates back to the simple periodic array of dipoles used by Läuger (1973), and the 'stylized' periodic helix introduced by Fischer et al. (1981). The MD simulation of Mackay et al. (1984) was the first to exploit the new possibilities in MD (McCammon et al. 1977), incorporating completely the flexibility of the channel molecule in all its atomic detail. On the basis of this detailed atomic model, Mackay et al. (unpublished observations) then went on to compute the free energy of $\mathrm{Cs}^{+}$at a few locations along the axis of the gA channel using FEP. A free-energy barrier of $+40 \mathrm{kcal} / \mathrm{mol}$ opposing permeation was found, most certainly as a result of the severe limitations in the accuracy of the potential function and in computational resources at the time. The results of their calculations was briefly reported in Jordan (1987). Unfortunately, the full manuscript entitled 'Free energy calculation for cesium ion transport along Gramicidin A' 


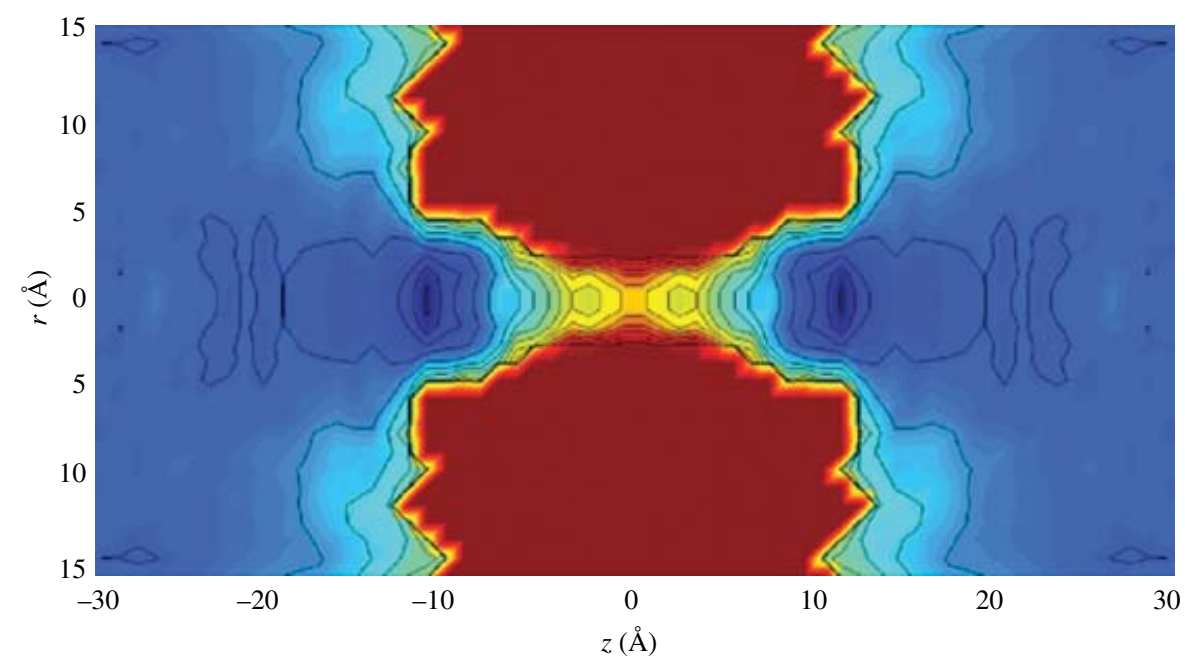

Fig. 11. The $2 \mathrm{D}$ PMF of a single $\mathrm{K}^{+}$ion as a function of axial position, $z$, and radial displacement, $r$, relative to a gramicidin A channel embedded in a DMPC bilayer (Allen et al. 2003b). The simulation system is shown in Fig. $2 a$. Contours are drawn at each $1 \mathrm{kcal} / \mathrm{mol}$. The PMF was calculated using umbrella-sampling simulations with 101 biasing windows.

by D. H. Mackay, P. M. Edelsten and K. R. Wilson (1985) remains unpublished. Nonetheless, it had a very wide circulation and had a profound impact on computational studies of ion permeation. In the two decades following this seminal work, there were several computational studies of the gA channel, aimed at addressing various questions (for a recent review see Roux, 2002a). The gA channel has become an important test case for theories of ion permeation, as will be discussed here. It is also an ideal prototypical benchmark system for investigating water permeability (Chiu et al. 1999a, b; deGroot et al. 2002) and the Grotthus-like mechanism of proton conduction along a single file of hydrogen-bonded water molecules (Nagle \& Morowitz, 1978; Pomes \& Roux, 1996, 1998; Sagnella \& Voth, 1996; Sagnella et al. 1996; Schumaker et al. 2000, 2001; Brewer et al. 2001), the effects of anesthetics (Tang \& Xu, 2002), and for testing the methodology used in the refinement of solid-state NMR structures (Ketchem et al. 1993; Kim et al. 2001; Allen et al. 2003a). Since the simulation of Woolf \& Roux (1994b), most MD simulations of $\mathrm{gA}$ have represented the membrane environment by explicitly including the phospholipid as well as the water molecules. However, it is only recently that progress in simulation methodology, force-field developments, and increased availability of inexpensive computers have made it possible to attempt to characterize ion permeation quantitatively, even for this simple channel.

\section{I.I Free-energy surface for $\mathrm{K}^{+}$permeation}

Rather than broadly summarize a large number of studies, we will focus our attention on the results of a recent study in which the complete free-energy landscape governing the passage of $\mathrm{K}^{+}$through the gA channel embedded in a lipid bilayer was characterized (Allen et al. 2003b). The 2D PMF $\mathcal{W}(z, r)$, function of both axial $(z)$ and radial $(r)$ coordinates of $\mathrm{K}^{+}$was calculated using umbrella-sampling techniques. A total of 101 window simulations were used where an ion was placed at regular positions along the z-axis and simulations were continued for 1 ns to 
ensure sufficient sampling for a converged PMF. The microscopic simulation system is shown in Fig. $2 a$, constructed initially from pre-equilibrated and pre-hydrated lipids using extensions of established techniques (Woolf \& Roux, 1996). Simulations were carried out with CHARMM (Brooks et al. 1983) using the PARAM27 force-field. As shown in Fig. 11, the full 2D PMF reveals a flat landscape in the bulk, far from the channel, the depth and position of binding sites at the channel entrances, the scale of the free-energy barrier that must be surmounted by the ion translocating across the narrow channel, and the extent of lateral motion of the ion. The value of the 2D PMF in the bulk region far from the channel is used to set the absolute reference for the complete free-energy surface. The free energy of an ion at the center of the channel relative to the bulk has been confirmed using FEP calculations (Allen et al. 2003b). Because the 2D PMF is determined in the laboratory frame, lateral movements of the ion and channel wobbling lead to fairly broad free energy wells near the channel entrances.

It is of interest to examine how the complete free-energy surface relates to the classical concept of a 'free-energy profile' $\mathcal{W}_{1 \mathrm{D}}(z)$, function of the position of the ion along the channel axis. Formally, it is defined from Eq. (97) by integrating out the lateral motions of the ion in the $x y$-plane. As observed from the 2D PMF shown in Fig. 11, the ion is only confined laterally when it is approximately within the range of $z$ from -15 to $+15 \AA$. Outside this region, the ion may be displaced to any extent in the $x y$-plane, and the concept of a free energy profile $\mathcal{W}_{1 \mathrm{D}}(z)$ along the channel axis is not valid. Furthermore, by virtue of Eq. (97), the value of $\mathcal{W}_{1 \mathrm{D}}(z)$ cannot be set with respect to some absolute reference [e.g. it is incorrect to assume that $\mathcal{W}_{1 \mathrm{D}}(z)$ is equal to zero for large $\mathrm{z}$. Consequently, the true depth of the binding sites relative to the bulk can be defined only via the complete free-energy surface provide by the 2D PMF. The 1D PMF of $\mathrm{K}^{+}$along the channel axis is shown in Fig. $12 a$, with the $\pm 15 \AA$ limits indicated as broken lines. In order to obtain a meaningful estimate of the free-energy profile some limitations and shortcomings of the simulations required further consideration in the calculation of Fig. $12 a$. First, a spurious destabilization of the ion in the channel arises from the finite size, the imposed periodicity and the heterogeneous polarity of the system (high dielectric water and low dielectric membrane). Secondly, some significant stabilization arising from the induced polarizability of the hydrocarbon chains of the lipid molecules is completely ignored because a non-polarizable force field is used. In the current non-polarizable model, the dielectric constant of the lipid chains is 1, whereas it should be closer to 2, the value for liquid hydrocarbons (Stern \& Feller, 2003). A continuum electrostatic approximation was used to correct for the artifacts caused by periodicity (Hunenberger \& McCammon, 1999) and lack of induced polarization of the lipids. Correcting for the spurious destabilization arising from the finite-size and periodicity of the simulated system leads to free energy of $-1.6 \mathrm{kcal} / \mathrm{mol}$ while correcting for the lack of polarization of the lipid chains leads to a further $-2 \cdot 1 \mathrm{kcal} / \mathrm{mol}$ stabilization (with the ion at the center of the channel). Changes in ionic concentration correspond to minor corrections, on the order of $0.2 \mathrm{kcal} / \mathrm{mol}$. The barrier in the $1 \mathrm{D}$ PMF (Fig. 12a), which has been corrected for these artifacts, is approximately $8 \mathrm{kcal} / \mathrm{mol}$ with respect to the binding site. The free energy of an ion at the deepest binding site at $z= \pm 11 \cdot 3 \AA$ is set to $-3.2 \mathrm{kcal} / \mathrm{mol}$ in this $1 \mathrm{D} \mathrm{PMF}$, to match the value in the full $2 \mathrm{D}$ PMF of Fig. 11. In addition to this deep outer binding site at $z=11.3 \AA$, there is a shallow well around $9.5 \AA$ (the inner binding site), and an additional three local free-energy minima inside each subunit of the gA dimer that contribute little to channel binding. The outer and inner binding sites have been shown to correspond to sites where a $\mathrm{K}^{+}$ ion is solvated by 3 and 2 water molecules respectively (Allen et al. 2003b). The main features of the $1 \mathrm{D}$ PMF of $\mathrm{K}^{+}$in $\mathrm{gA}$, shown in Fig. $12 a$, are in qualitative agreement with other calculations 

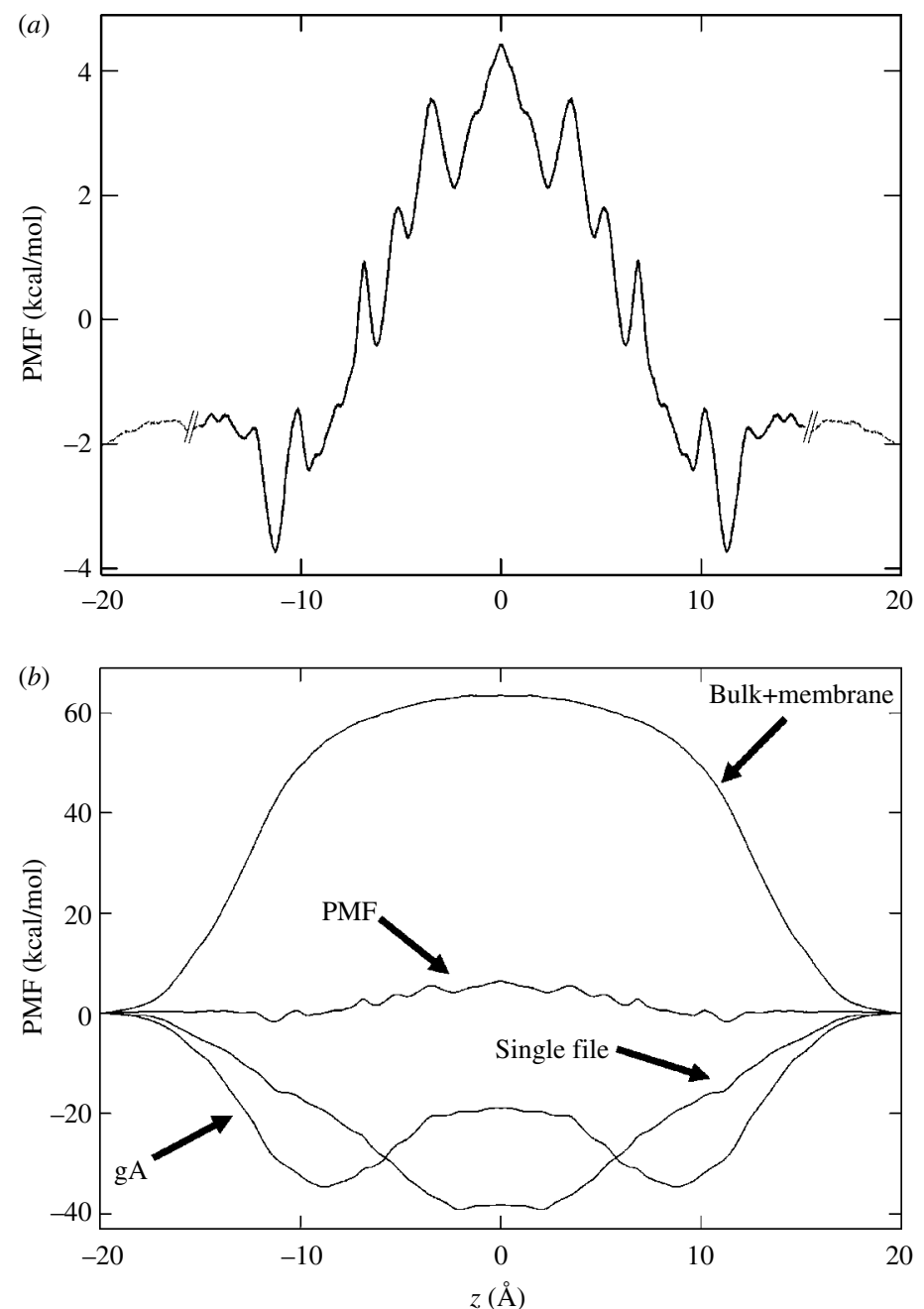

Fig. 12. (a) Free-energy profile, or 1D PMF, of $\mathrm{K}^{+}$going through the gA channel (Allen et al. 2003b). Broken lines at $|₹|=15 \AA$ indicate that the 1D PMF is not rigorously defined beyond those points. The PMF has been corrected for size, membrane dielectric constant and concentration effects (see text). (b) Mean force decomposition. The corrected 1D PMF and contributions from the gA dimer and single-file water are plotted with the remaining contribution from the bulk electrolyte and membrane (with corrections).

(Allen et al. 2003c), although there are significant differences because a non-Boltzmann averaging of free-energy profiles calculated with a dragging procedure was used in those simulations, which introduced large uncertainties.

It is known that monovalent cations partition spontaneously into the channel, although the equilibrium binding constant of the monovalent cations is relatively weak (Hinton et al. 1988). The one-ion equilibrium binding constant can be calculated from Eq. (79) using the PMF $\mathcal{W}(\mathbf{r})$. The equilibrium dissociation constant for the entire channel is $0.34 \mathrm{M}$, within the range of reported experimental values determined from NMR and conductance studies on gA: 0.017 M ( Jing et al. 1995) and 0.019-0.73 m (Hinton et al. 1986). This suggests that the large solvation free energy of the ion in bulk water is adequately compensated by the interactions with the $\mathrm{gA}$ in the MD model. 


\subsubsection{Mean-force decomposition}

The mean-force decomposition expressed in Eq. (105) is a useful technique to resolve particular contributions to the total free-energy profile. The mean-force decomposition of the 1D PMF is shown in Fig. 12 b. Obviously, the seemingly flat PMF with its relatively small free-energy barrier arises from the cancellation of very large opposing contributions from protein, single-file water and the remaining bulk electrolyte and membrane. In this respect, it is easy to understand how the PMF can be in error by a few $\mathrm{kcal} / \mathrm{mol}$. The contribution from the membrane and bulk electrolyte is $63.5 \mathrm{kcal} / \mathrm{mol}$, roughly as expected for the Born energy barrier corresponding to the transfer of a $\mathrm{K}^{+}$from bulk solution to the center of a membrane slab with dielectric constant of 1 (Parsegian, 1969; Levitt, 1978; Jordan, 1981). This barrier is eliminated, almost completely, by interactions with the protein and single-file water. The most striking observation is that the single-file water itself accounts for $-39 \cdot 2 \mathrm{kcal} / \mathrm{mol}$ stabilization, i.e. nearly half of the $-80 \mathrm{kcal} / \mathrm{mol}$ solvation free energy of $\mathrm{K}^{+}$in bulk water (Cox et al. 1974). The importance of this contribution, which was conjectured by Wilson and co-workers nearly 20 years ago (Mackay et al. 1984; Mackay \& Wilson, 1986), is surprising given that only two water molecules are in direct contact with the ion in the narrow pore. For comparison, a FEP calculation (with the same potential function) shows that the solvation free energy of $\mathrm{K}^{+}$surrounded by its first hydration shell of eight water molecules is about $-46 \mathrm{kcal} / \mathrm{mol}$ (Roux et al. 2000). As suggested by the results obtained from the semi-microscopic models developed by Jordan and co-workers (Partenskii \& Jordan, 1992a, b; Dorman et al. 1996), it is likely that the restricted orientational freedom of water molecules aligned in single file is the cause of this phenomenon.

\subsubsection{Cation-binding sites}

The location of the cation-binding sites, at the $\mathrm{C}$ terminus of the monomers, has been deduced indirectly from various experimental measurements (Urry et al. 1982; Smith et al. 1990a; Olah et al. 1991; Andersen \& Koeppe, 1992; Woolf \& Roux, 1997). Nonetheless, the results from these various sources is remarkably consistent: analysis of ion-flux data indicates that the cation in the binding site is $14 \%$ into the transmembrane potential, corresponding to $9 \AA$ using the constant field assumption (Andersen \& Koeppe, 1992); low-angle X-ray scattering on gA incorporated in oriented bilayers shows that $\mathrm{Tl}^{+}$binds at $9 \cdot 6 \pm 0 \cdot 3 \AA$ from the center of the channel, and $\mathrm{K}^{+}$also binds predominantly at this site, though slightly further from the center of the channel (Olah et al. 1991); analysis of solid-state $\mathrm{NMR}{ }^{13} \mathrm{C}$ and ${ }^{15} \mathrm{~N}$ chemical shift anisotropy data (Smith et al. 1990b; Tian et al. 1996), using MD simulations, indicate that $\mathrm{Na}^{+}$binds at $9 \cdot 2 \AA$ (Woolf \& Roux, 1997). Therefore, the main cation-binding site is the inner site according to numerous experimental sources. In contradiction with the experimental results, the outer site is slightly deeper than the inner site in the current PMF (Allen et al. 2003b). Clearly, the relative depth of the inner and outer cation-binding sites near the entrance of the gA channel is very sensitive to the details of the potential function. Previous calculations have found that either the inner (Roux \& Karplus, 1993; Roux et al. 1995) or outer (Woolf \& Roux, 1997; Allen et al. 2003b) binding site was the most stable position. Overall, there are no large structural distortions of the channel due to the presence of the ion. In the inner-binding site, the main channel ligand is provided by the carbonyl group of the Leu10-Trp11 peptide linkage, which exhibits the largest deflection relative to the ion-free channel structure. This is in the order of 


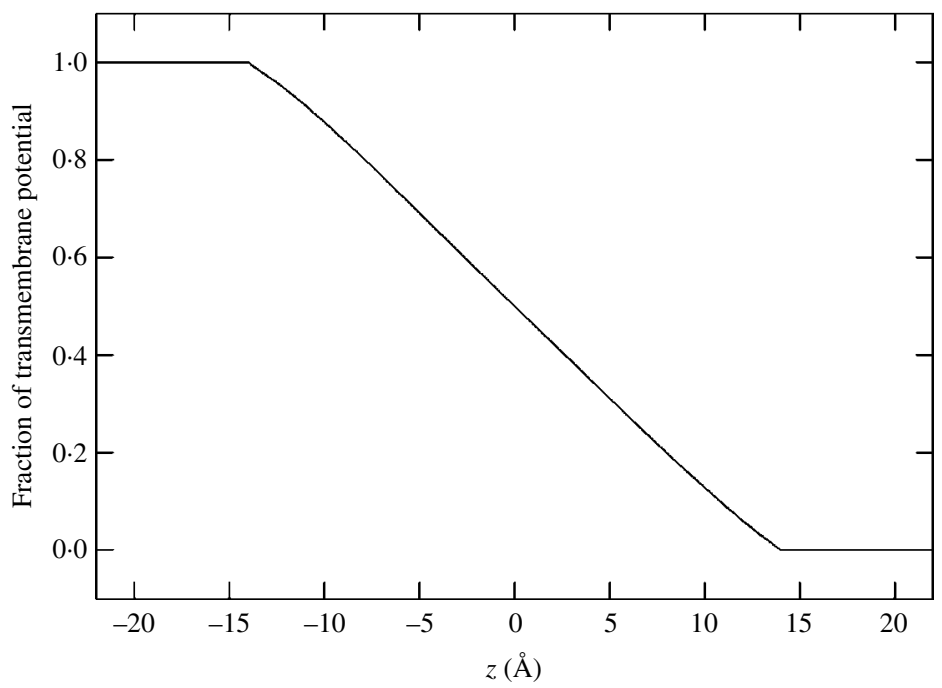

Fig. 13. Transmembrane voltage profile across the gA channel.

10 to 15 degrees for $\mathrm{Na}^{+}$(Woolf \& Roux, 1997) and only 5 degrees for $\mathrm{K}^{+}$(T. W. Allen, O. S. Andersen and B. Roux unpublished results). According to the mean force decomposition, the inner- and outer-binding sites in the PMF are seen to arise from a delicate interplay between water and protein interactions. Figure $12 b$ shows that the inner-binding site (the dominant binding site experimentally) arises from the mean force exerted by the protein. It has often been assumed that the inner-binding sites, near the channel entrances owe their existence to a superposition of a broad electrostatic ion-membrane image repulsion Fig. 6 and a short-range ion-channel attraction (Andersen \& Procopio, 1980; Finkelstein \& Andersen, 1981). Interestingly, in the $\beta$-helical structure, Leu10 corresponds to the first backbone carbonyl oxygen pointing toward the bulk solvent which is not forming a backbone-backbone hydrogen bond. For this reason, only the carbonyls on this linkage, or further away from the channel center, are able to provide ligands for a cation without loosing backbone hydrogen bonds.

\section{I.4 Channel conductance}

To ascertain the magnitude of the current that can pass through the channel, the net stationary flux of ions across the channel was calculated using the calculated single ion equilibrium PMF and a 1D-NP theory (Levitt, 1986). Two additional ingredients are needed: the transmembrane potential and the diffusion coefficient along the channel axis. The transmembrane potential along the gA channel was calculated by solving Eq. (82) with a finite-difference method implemented in the PBEQ module (Im et al. 1998) of CHARMM (Brooks et al. 1983). In the calculation, the channel and the membrane were represented in full atomic detail, while the electrolyte was represented as a uniform dielectric constant of 80 with a salt concentration of $1 \mathrm{~m}$. The calculated transmembrane potential field along the channel axis is shown in Fig. 13. The field is linear, except for small deviations near the entrance of the channel. The inner-binding site, $\sim 9.5 \AA$ at the entrance of the channel, is at $14.5 \%$ of the transmembrane potential, in excellent agreement with ion-flux measurements (Andersen \& Koeppe, 1992). The diffusion coefficient $D(z)$ was extracted from the Laplace transform of the velocity autocorrelation 


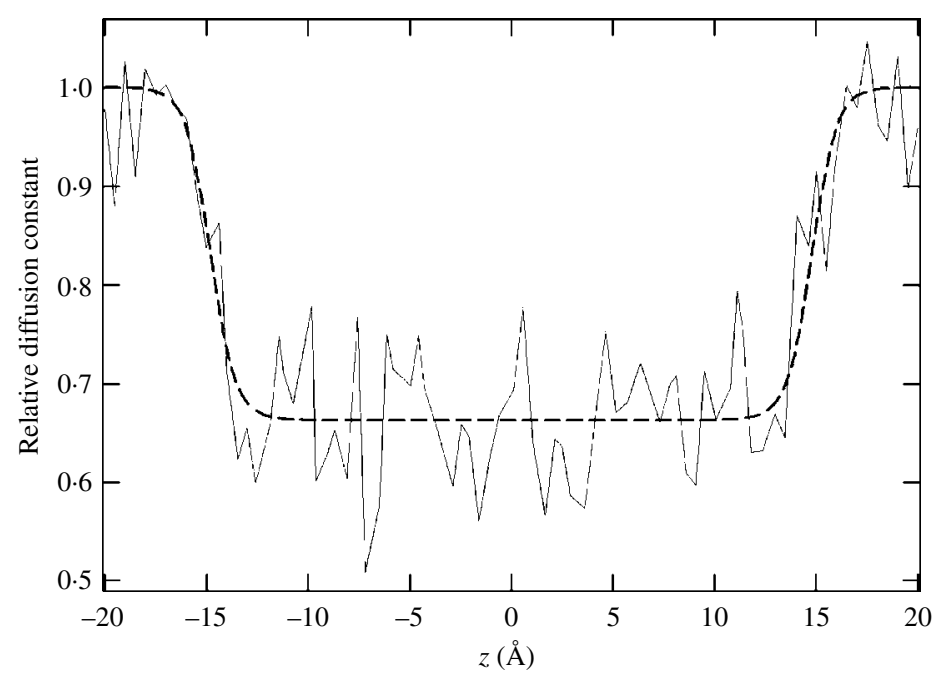

Fig. 14. $\mathrm{K}^{+}$ion diffusion profile. Calculated values of the axial component of the ion diffusion coefficient, for each window simulation, are plotted with a solid line. All values have been scaled relative to the calculated bulk value of $0.37 \AA^{2} / \mathrm{ps}$. The sigmoidal fit is drawn as a dashed curve which drops from 1 to $0 \cdot 67$ inside the channel.

function calculated from the umbrella-sampling simulations using an analysis based on the GLE for non-uniform systems Eq. (113). The diffusion coefficient profile, which is shown in Fig. 14, reveals that the diffusion of the ion within the channel drops to about $67 \%$ of its value in the bulk. That the diffusion coefficient remains near bulk levels throughout the channel, suggests that ion permeation through the gA channel is governed by the features of the PMF rather than by dynamical and dissipative factors.

To assess the ability of the computational model to reproduce the magnitude of the ion flux observed experimentally, we only consider the maximum single-channel conductance $g_{\max }$, calculated from Eq. (118). This calculation may be summarized using the acronyms given in Table 1 as $1 \mathrm{D}-\mathrm{NP} /[\mathrm{PMF}(\mathrm{MD})+\mathrm{Vmp}(\mathrm{PB}-\mathrm{V}, \mathrm{MD})]$, to indicate that a $1 \mathrm{D}-\mathrm{NP}$ diffusion theory is used with a PMF calculated from all-atom MD and a transmembrane potential calculated from the PB-V equation used in Eq. (90). This analysis provides a rough estimate because the 1-ion PMF is extended into the regime of large concentration, ignoring the changes in conduction that could be associated with multiple ion occupancy (Roux et al. 1995). The length of the pore $L$, corresponds to the diameter of the sphere defining the single ion region (chosen to be $28 \AA)$ and is within the range where the $1 \mathrm{D}$ PMF is meaningful $(|z|<15 \AA)$. The PMF leads to a maximum conductance of $0.8 \mathrm{pS}$, approaching the experimental value of $24 \mathrm{pS}$ better than any previous MD study of $\mathrm{gA}$. The agreement is encouraging because the discrepancy could be accounted for by only small changes to the PMF; reducing the barrier by less than $3 \mathrm{kcal} / \mathrm{mol}$ reproduces the experimental $g_{\max }$. This demonstrates that a detailed all-atom force-field is able (in principle) to describe the energetics of ion permeation through the gA channel with at least semi-quantitative accuracy, with minor changes to the ion-carbonyl Lennard-Jones parameter. It can be expected that including electronic polarization from the protein, water and lipid molecules, will be needed to reach quantitative agreement with experiment. 


\subsubsection{Selectivity}

The agreement with experimental dissociation and conductance measurements is promising and indicative of the overall correctness of the computational method. Ultimately, it will be of interest to elucidate the factors governing the selectivity of the gA channel using a similar computational approach. For example, although the gA channel is electrically neutral, it is ideally selective for small univalent cations, as it is blocked by divalent cations and completely impermeable to anions (Andersen \& Koeppe, 1992). It was shown previously, on the basis of alchemical FEP simulations, that $\mathrm{Cl}^{-}$has a much lower affinity for the interior of the $\mathrm{gA}$ channel than $\mathrm{K}^{+}$(Roux, 1996) even though these two ions have similar solvation free energies in bulk water (approximately $-80 \mathrm{kcal} / \mathrm{mol}$ ). The cation specificity of the $\mathrm{gA}$ arises from the strong asymmetry in the permanent charge distribution of the peptide backbone, a mechanism that was first proposed by Urry (1971). In simple terms, the backbone carbonyl CO group can compensate favorably for the loss of hydration by a cation, whereas the corresponding interaction between the backbone amide group $\mathrm{NH}$ and anions are not as favorable. One may therefore anticipate that the PMF of anions will present a very large free-energy barrier opposing their passage through the channel. The factors governing the exclusion of divalent cations could be elucidated by similar calculations.

\subsection{Computational studies of KcsA}

The structure of KcsA, the first one available at atomic resolution for a $\mathrm{K}^{+}$channel triggered a large number of computational studies based on MD (Allen et al. 1999, 2000; Guidoni et al. 1999, 2000; Åqvist \& Luzhkov, 2000; Bernèche \& Roux, 2000, 2001; Capener et al. 2000; Luzhkov \& Åqvist, 2000, 2001a, b; Shrivastava \& Sansom, 2000; Biggin et al. 2001; Crouzy et al. 2001), PB (Roux \& MacKinnon, 1999; Roux et al. 2000; Ranatunga et al. 2001), and BD (Allen \& Chung, 2001; Mashl et al. 2001; Burykin et al. 2002, 2003). Because of the moderate resolution of the X-ray data $(3 \cdot 2 \AA)$, the computational studies initiated from the original 1998 KcsA structure (pdb id 1BL8) had to proceed with a limited amount of information. In particular, the exact number and configurations of the ions and water molecules in the selectivity filter was not known, the conformation and ionization state of Glu71 near the selectivity filter could not be resolved, and whether the channel was in an open or closed state in the crystal structure was unclear.

The picture was rapidly refined during the following few years, as additional structural and functional information became available. X-ray structures of the KcsA channel were obtained at higher resolution (up to $2 \cdot 0 \AA$ ) (Morais-Cabral et al. 2001; Zhou et al. 2001a, b), followed by structures for other $\mathrm{K}^{+}$channels: MthK $(3 \cdot 4 \AA)$, a calcium-activated channel crystallized in

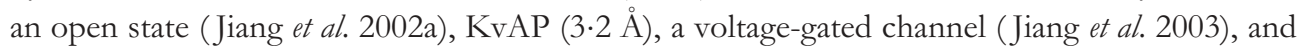
KirBAC (3.65 $\AA$ ), an inward rectifier (Kuo et al. 2003). Further information about the molecular movements of the transmembrane helices of KcsA and their role in channel gating was obtained by electron paramagnetic resonance (EPR) (Gross et al. 1999; Cortes et al. 2001; Liu et al. 2001; Gross \& Hubbell, 2002) and mass spectroscopy (Kelly \& Gross, 2003). At the same time, the results of electrophysiological experiments on KcsA became available (Heginbotham et al. 1999; LeMasurier et al. 2001; Nimigean \& Miller, 2002; Kutluay et al. In Press). In retrospect, it is encouraging that many results from the computational studies were consistent with the rapidly emerging data, sometimes even in advance of the fact. As emphasized by Miller (2001), these 


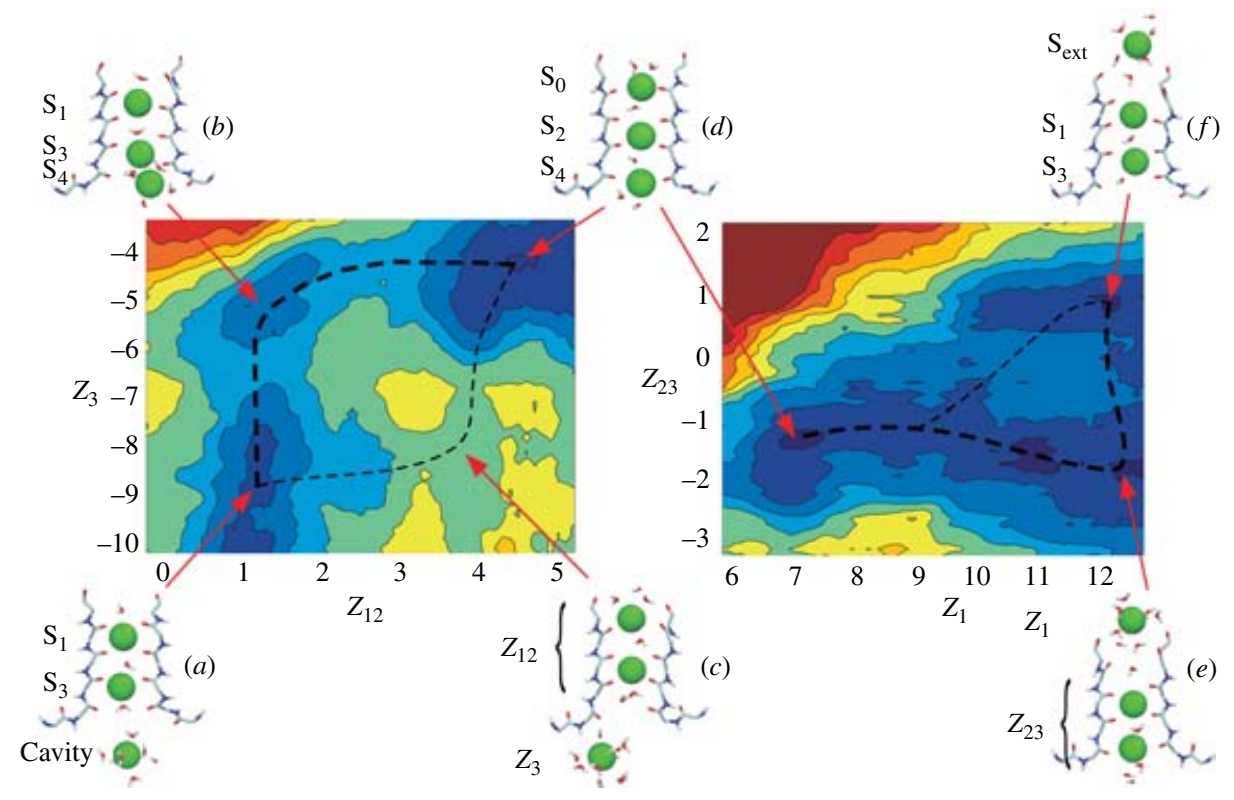

Fig. 15. Topographic free-energy maps of ion conduction calculated from umbrella-sampling molecular dynamics simulations. Each color level corresponds to an energy of $1 \mathrm{kcal} / \mathrm{mol}$; the axes are given in angstroms. The position of the ions (numbered in successive order from 1 to 3 starting from the ion on the extracellular side), $Z_{1}, Z_{2}$, and $Z_{3}$ are defined relative to the center-of-mass of the backbone atoms of residues Thr75-Val76-Gly77-Tyr78 which constitute the central core of the selectivity filter. The definition of the reduced reaction coordinates $\left(Z_{12}, Z_{3}\right)$ and $\left(Z_{1}, Z_{23}\right)$ is indicated in $(c)$ and $(e)$, with $Z_{i j}$ corresponding to the center of mass of ions $i$ and $j$. A direct comparison of the energy levels in the two 2D free maps is meaningful. The lowest energy pathway (highlighted by a dotted line) follows the configurations $a-b-d-f$ [a spontaneous transition along this pathway has been observed previously during an unbiased simulation of the KcsA channel (Bernèche \& Roux, 2000)]. A secondary pathway (highlighted by a thin dashed line) following the configurations $a-c-d-f$ is also possible.

successful predictions reinforce the confidence in computational studies of ion channels based on atomic models.

\subsection{Multi-ion free-energy surface and cation-binding sites}

To characterize the ion conduction process, Bernèche \& Roux (2001) calculated the complete multi-ion free-energy surface governing the movement of $\mathrm{K}^{+}$in the pore of KcsA using MD umbrella-sampling simulations. The atomic simulation system at the basis of this work is shown in Fig. $2 b$. The results of the calculations are shown in Fig. 15. For the sake of visualization the results are presented as two-dimensional $(2 d)$ topographic maps of the free-energy landscape governing the ion conduction through the selectivity filter of the $\mathrm{K}^{+}$channel. To produce the $2 \mathrm{D}$ maps, the full free-energy function $\mathcal{W}\left(z_{1}, z_{2}, z_{3}\right)$, which depends on the position of the three ions along the channel axis has been projected onto two different planes with reduced reaction coordinates (see caption of Fig. 15). Typical configurations of the selectivity filter with the three $\mathrm{K}^{+}$permeating ions and nearby water molecules labeled (a) to $(f)$ are given in Fig. 15 to illustrate the elementary steps along the ion conduction process. The free-energy barrier separating these configurations is in the order of $2-3 \mathrm{kcal} / \mathrm{mol}$. 


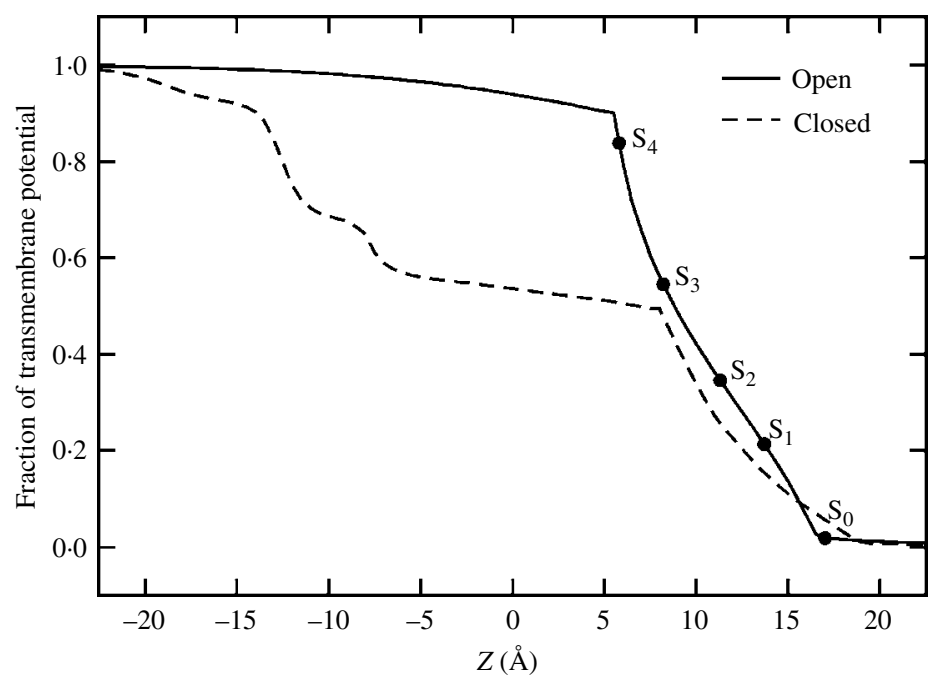

Fig. 16. Transmembrane potential profile along the pore of an open-state model of the KcsA channel (Doyle et al. 1998) generated from the structure of the MthK channel (Jiang et al. 2002a). The curve is drawn assuming a positive unitary value of the intracellular potential. The potential at the position of the five most important cation binding sites, $\mathrm{S}_{0}, \mathrm{~S}_{1}, \mathrm{~S}_{2}, \mathrm{~S}_{3}$ and $\mathrm{S}_{4}$, is highlighted (•). For comparison, the profile calculated for the closed state of KcsA is also shown (Roux et al. 2000).

Even though no large free-energy barriers opposing ion conduction are observed in the freeenergy maps, five position along the permeation pathway are preferably occupied by $\mathrm{K}^{+}$ions, hereafter referred as $S_{0}, S_{1}, S_{2}, S_{3}$, and $S_{4}$, from the extracellular side of the selectivity filter. Those are illustrated in Fig. $10 b$. Ions located in $\mathrm{S}_{1}, \mathrm{~S}_{2}$ or $\mathrm{S}_{3}$, are in contact with the main chain carbonyl oxygens $\left(\mathrm{S}_{1}\right.$ : Gly77 and Tyr78; $\mathrm{S}_{2}$ : Val76 and Gly $77 ; \mathrm{S}_{3}$ : Thr75 and Val76) and only 1-2 water molecules. The ion in $\mathrm{S}_{4}$ is hydrated by $2-3$ water molecules and making intermittent contacts with the carbonyl oxygens and the side-chain of Thr 75 while the ion in site $\mathrm{S}_{0}$ is hydrated by 3-4 water molecules and making some contacts with the carbonyl oxygens of Tyr78. The multi-ion free-energy surface was calculated on the basis of the X-ray structure at $3 \cdot 2 \AA$ resolution of Doyle et al. (1998). It reproduces the four cation-binding sites $\left(\mathrm{S}_{1}, \mathrm{~S}_{2}, \mathrm{~S}_{3}\right.$ and $\left.\mathrm{S}_{4}\right)$ located in the narrow selectivity filter, which were already known (Doyle et al. 1998), and also predicted the two additional sites $\left(\mathrm{S}_{\text {ext }}\right.$ and $\mathrm{S}_{0}$ ) located on the extracellular side of the channel, which were observed at $2 \cdot 0 \AA$ resolution (Zhou et al. 2001b). A concerted multi-ion transition leading to a configuration in which a $\mathrm{K}^{+}$was bound in the site $\mathrm{S}_{0}$ had also been previously observed during MD simulations (Bernèche \& Roux, 2000).

\subsubsection{Channel conductance}

The full free-energy function $\mathcal{W}\left(z_{1}, z_{2}, z_{3}\right)$ provides the essential information to describe the conduction mechanism in the pore of KcsA (i.e. single-file diffusion of the ions with water in between). The absence of large energy barriers is traditionally interpreted as a strong evidence that the ion conduction process ought to be essentially diffusion limited. But what could be the throughput of $\mathrm{K}^{+}$across the selectivity filter? Such a question cannot be addressed without further considerations about dynamical processes. To simulate ion conduction through the selectivity filter of KcsA, we constructed a 'realistic' BD model using the PMF calculated from all-atom MD (Bernèche \& Roux, 2003). Two additional ingredients are needed to simulate 


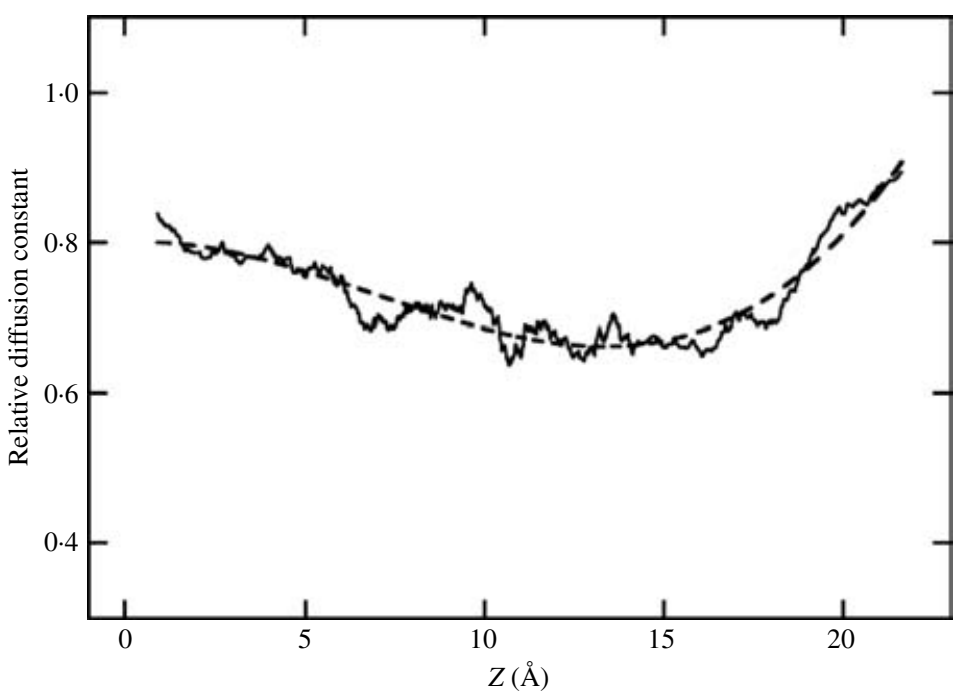

Fig. 17. Diffusion coefficient of $\mathrm{K}^{+}$relative to its value in the bulk as a function of the position along the channel axis. An analytical form fitted to the results from the simulations was used in the Brownian dynamics trajectories (dashed line). The absolute value of the calculated diffusion coefficient in bulk solution $\left(0.37 \AA^{2} / \mathrm{ps}\right.$ ) was uniformly scaled to the corresponding experimental value of $0 \cdot 185 \AA^{2} / \mathrm{ps}$ (Mills \& Lobo, 1989) in order to account for the underestimated viscosity of the TIP3P water model (Feller et al. 1996) at the temperature of the molecular dynamics simulations (330 K) (Bernèche \& Roux, 2001).

non-equilibrium ion conduction: the transmembrane potential and the diffusion coefficient along the channel axis. The transmembrane potential was calculated for a model of the KcsA channel in the open state constructed on the basis of the X-ray structure of the calcium-activated MthK channel (Jiang et al. 2002a) using the PB-V Eq. (82). The result is shown in Fig. 16. For comparison, the voltage profile along the axis of the channel in the closed state is also shown (Roux et al. 2000). In accord with previous results (Roux et al. 2000; Jiang et al. 2002b), the variation in the transmembrane potential difference becomes increasingly localized across the selectivity filter as the channel opens. The main cation binding sites $\left(\mathrm{S}_{0}-\mathrm{S}_{4}\right)$ are located in the region where variation in the transmembrane potential is the steepest, while the potential difference across the wide aqueous intracellular vestibule is very small. The diffusion coefficient $D(z)$ was extracted from the umbrella-sampling simulations using an analysis based on the GLE for non-uniform systems. (Berne et al. 1988; Straub et al. 1990; Crouzy et al. 1994). The calculated diffusion coefficient varies weakly throughout the entire permeation pathway, decreasing to roughly $70 \%$ of its bulk value in the selectivity filter region (see Fig. 17). Because there are no abrupt variations in $D(z)$, the character of the random ion movements governing the conduction mechanism is largely determined by the structure of the multi-ion free-energy surface $\mathcal{W}$ rather than the dissipative and frictional forces. This model may be summarized using the acronyms given in Table 1 as $\mathrm{BD} /[\mathrm{PMF}(\mathrm{MD})+\mathrm{Vmp}(\mathrm{PB}-\mathrm{V})]$, to indicate that $\mathrm{BD}$ trajectories are generated using a PMF that was calculated with all-atom MD and a transmembrane potential that was calculated with Eq. (91).

Several BD trajectories on the $\mu$ s time-scale were generated to simulate ion conduction under various non-equilibrium conditions of ion concentration and transmembrane voltage. The $\mathrm{BD}$ trajectories were generated using a discrete-state Markov chain framework model (Bernèche \& Roux, 2003). As an illustration, a 100 ns segment from a typical BD trajectory corresponding 


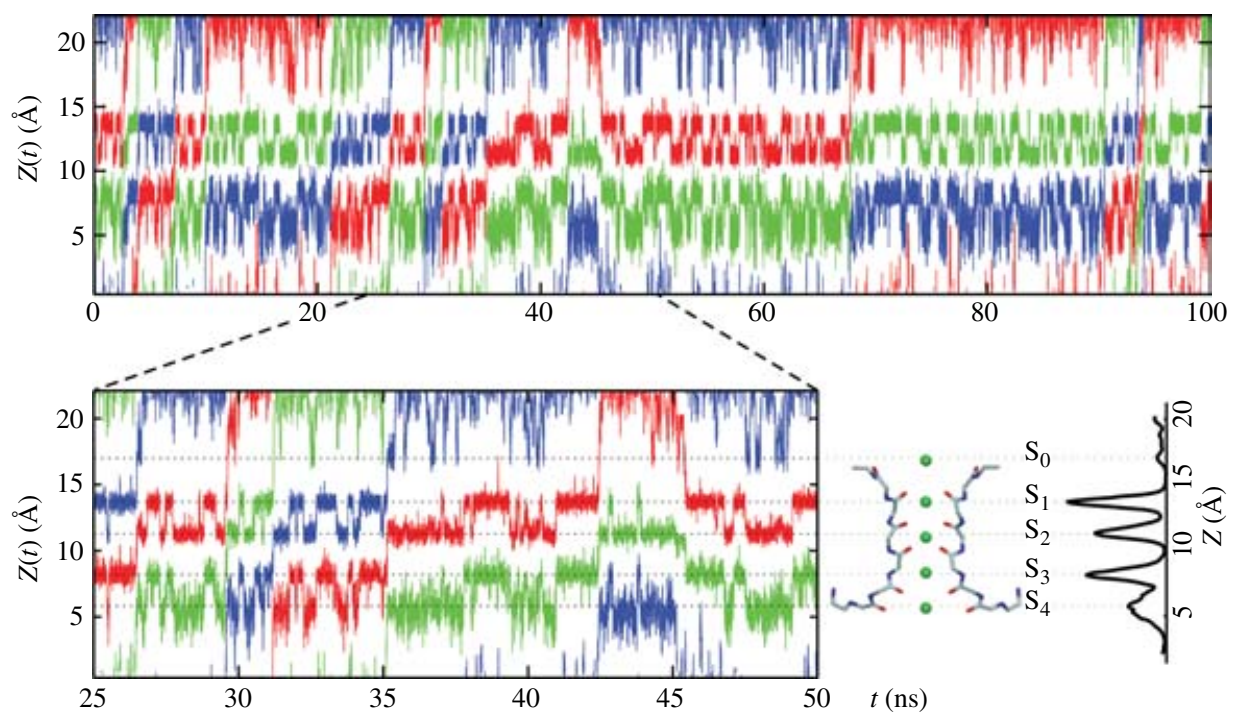

Fig. 18. Typical Brownian dynamics trajectory generated with an applied membrane potential of $+50 \mathrm{mV}$ and under symmetric conditions of $\mathrm{K}^{+}$concentration chosen to yield a channel occupied by 3 ions $50 \%$ of the time. The $Z(t)$ of the 2 or 3 ions in the system is alternatively plotted in blue, red and green for the sake of clarity. The relative ion density along the pore is shown in relation to the different binding sites. Many outward translocation events can be observed; for example, the red curve shows some of these events between 5 and 10 ns, 25 and 30 ns, 35 and 45 ns, 90 and 95 ns. A re-entry of a translocating ion in the direction opposite to the transmembrane potential can be observed between 45 and 68 ns. Binding of an ion from the extracellular solution briefly to the site $\mathrm{S}_{0}$ stabilizes ions in sites $\mathrm{S}_{2}$ and $\mathrm{S}_{4}$ (e.g. around $t=48 \mathrm{~ns})$.

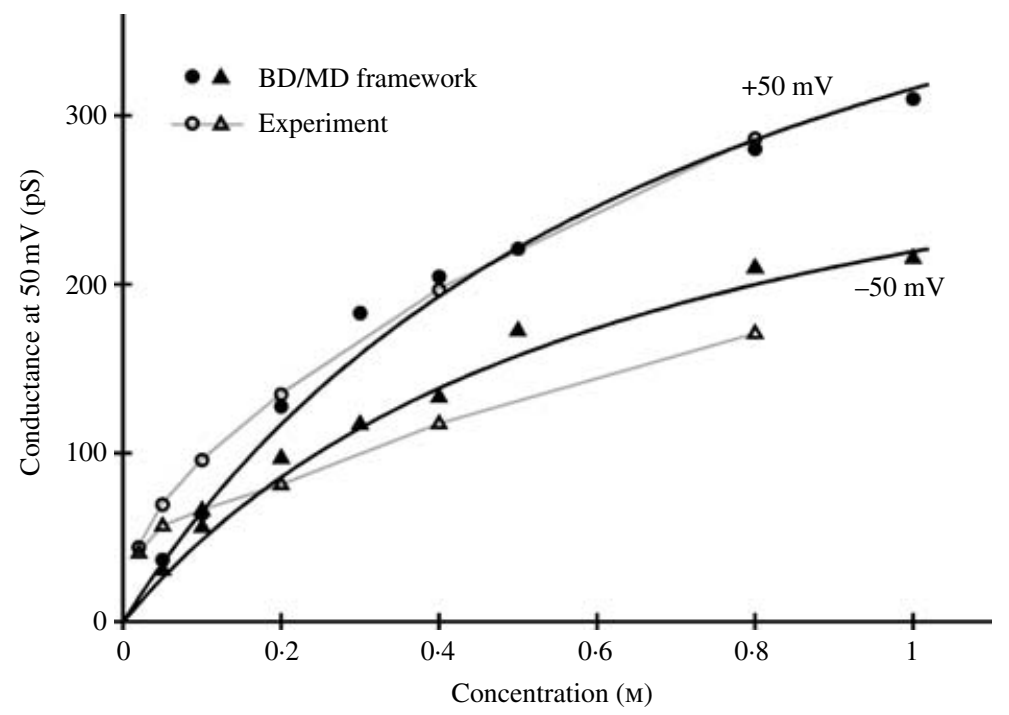

Fig. 19. (a) Current-voltage $(I-V)$ relation calculated from Brownian dynamics simulations under symmetric conditions and $\mathrm{K}^{+}$concentration of $400 \mathrm{~mm}$. (b) Conductance of the KcsA channel at $\pm 50 \mathrm{mV}$ as a function of permeant ion concentration. The variation of the channel conductance as a function of $\mathrm{K}^{+}$ concentration follows a first-order saturation with $K_{\mathrm{D}}$ of 740 and $640 \mathrm{~mm}$, for +50 and $-50 \mathrm{mV}$ respectively. Experimental data from LeMasurier et al. (2001) (open symbols and thin gray lines) were taken from: http://www.jgp.org/cgi/content/full/118/3/303/DC1/1. 
to the evolution of the position of the three ions along the pore with an applied transmembrane potential of $+100 \mathrm{mV}$ is shown in Fig. 18. During the BD trajectories, the ions stay in welldefined states for some time, followed by sudden concerted 'hopping' transitions occurring in a random fashion. The relation of the $\mathrm{BD}$ trajectory to the average density of ions along the pore axis is illustrated schematically in the bottom part of Fig. 18 (right). Because the trajectories were generated using a PMF calculated from all-atom MD, the dominant positions of the $\mathrm{K}^{+}$during the trajectories are consistent with the cation-binding sites observed in the pore (Bernèche \& Roux, 2001; Zhou et al. 2001b).

The calculated conductance as a function of ion concentration is shown in Fig. 19 and compared with the experimental results from LeMasurier et al. (2001). The calculated conductance $g$ exhibits a typical first-order saturation, which is expected because the model represents ion conduction solely in terms of two- and three-ion occupied states. The observed deviations from the first-order behavior in the experimental data at low concentration could reflect the onset of a different ion conduction mechanism with less than $3 \mathrm{~K}^{+}$. The values of $g_{\max }$, the maximum conductance of the channel at saturating concentration, estimated from Fig. 19 is in the order of $550 \mathrm{pS}$ and $360 \mathrm{pS}$ for outward and inward ions flux respectively. These values are in remarkable agreement with the experimental measurements (LeMasurier et al. 2001). The calculated $g_{\max }$ correspond to maximum $\mathrm{K}^{+}$throughput of the pore, extending from the center of the intracellular cavity to the external binding site, $\mathrm{S}_{\text {ext }}$. Incorporating diffusion-limited access resistances at the ends of the pore (not taken into account in the current simulations) would only decrease the $g_{\max }$. The variation of the conductance as a function of the ion concentration depends sensitively on the equilibrium factors that governs the occupancy of the pore. In the model, the free-energy difference between 2 and $3 \mathrm{~K}^{+}$in the pore was empirically set to yield a pore occupied by $3 \mathrm{~K}^{+}$ions $50 \%$ of the time at $800 \mathrm{~mm}$, in accord with experimental observations (LeMasurier et al. 2001). Nonetheless, no adjustable parameter affects the calculated $g_{\max }$ itself. For instance, it is independent of the value of the equilibrium-binding constant, the value of the cross-sectional area, and the details of the exit and entry steps as modeled in the BD by Eqs. (121) and (122). The magnitude of $g_{\max }$ corresponds to the maximum throughput that the selectivity filter can sustain.

\subsubsection{Mechanism of ion conduction}

A few typical events resulting in the net translocation of $1 \mathrm{~K}^{+}$can be observed in Fig. 18 (see caption). Such 'productive' translocation events frequently occur according to a particular pattern during which the five cation-binding sites $\left(\mathrm{S}_{0}, \mathrm{~S}_{1}, \mathrm{~S}_{2}, \mathrm{~S}_{3}, \mathrm{~S}_{4}\right)$ are occupied by three ions for a very brief period of time. Such configurations, with alternatingly 2 and $3 \mathrm{~K}^{+}$ions in the selectivity filter, are reminiscent of the discrete states postulated in kinetic rate models of multiion channels (Hille \& Schwarz, 1978). The elementary microscopic events leading to outward ion conduction can be summarized as follow. For extensive periods of time, the selectivity filter is occupied by $2 \mathrm{~K}^{+}$ions located alternatively in the $\mathrm{S}_{1}$ and $\mathrm{S}_{3}$ sites, or the $\mathrm{S}_{2}$ and $\mathrm{S}_{4}$ sites, while a third ion frequently attempts to enter the channel on the intracellular side or the extracellular side. The exchange between the configurations $\left[\mathrm{S}_{1}, \mathrm{~S}_{3}\right]$ and $\left[\mathrm{S}_{2}, \mathrm{~S}_{4}\right]$, which occurs on a time-scale of $\sim 1-2$ ns, corresponds to a rapid but non-productive 'back-and-forth shuttling' of the $2 \mathrm{~K}^{+}$ in the selectivity filter. While the two ions are located in the sites $S_{1}$ and $S_{3}$, a third ion hops from the intracellular vestibule into the site $S_{4}$ and collides with the ion in $S_{3}$. This induces a concerted transition to a state in which the three ions occupy sites $S_{4}, S_{2}$ and $S_{0}$. This is then followed by 
the rapid dissociation and departure of the outermost ions in $\mathrm{S}_{0}$ on the extracellular side, yielding the conduction of one net charge.

This process leading to productive translocation events is qualitatively similar to the 'knock-on' mechanism proposed by Hodgkin \& Keynes (1955). A typical 'knock-on' event can be observed in Fig. 18 between $t=26$ ns and $t=35$ ns. The details of the ion conduction process does, however, present a challenge to our understanding of repulsive forces at play in multi-ion systems. The 'knock on' mechanism is possible because simultaneous occupancy of the adjacent sites $\mathrm{S}_{3}$ and $\mathrm{S}_{4}$ is not energetically prohibitive while the dissociation of the outermost ion in $\mathrm{S}_{0}$ is accelerated by ion-ion repulsion (Bernèche \& Roux, 2001). When the two ions are in $S_{4}$ and $S_{3}$, the 'bare' electrostatic energy is $+83 \mathrm{kcal} / \mathrm{mol}$ and the mutual repulsive coulombic force between the $2 \mathrm{~K}^{+}$ions corresponds to an electric field of $9 \times 10^{9} \mathrm{~V} / \mathrm{m}$, which is equivalent to the field produced by a transmembrane potential of $27 \mathrm{~V}$ ! Yet, according to the PMF shown in Fig. 15, the free energy of this state is only $1 \mathrm{kcal} / \mathrm{mol}$ higher than a state in which the ions occupy the cavity and site $\mathrm{S}_{3}$. Similar collisions between two ions occupying adjacent sites can also briefly occur in the selectivity filter. For example, the back-and-forth shuttling process of the two ions in the binding sites is typically initiated by a brief transition to a state with two ions in adjacent sites $S_{2}$ and $S_{3}$ (however, simultaneous occupancy of the adjacent sites $S_{1}$ and $S_{2}$ is energetically unfavorable and is not observed). Such rapid collisions suggest that the effective electrostatic repulsion between the ions in the filter is manifested mostly at short distances. Though, without the repulsion from the other two incoming ions, the outermost ion would be tightly bound to its site and its exit toward the extracellular side would require a significant activation of free energy. Therefore, repulsive forces are absolutely essential for rapid conduction.

\subsubsection{Selectivity}

The calculations show that the conduction of $\mathrm{K}^{+}$ions in $\mathrm{Kcs}$ A is extremely rapid, but can the model also show that the channel remains at the same time selective for $\mathrm{K}^{+}$over $\mathrm{Na}^{+}$? Selectivity arises primarily from the relative free energy of $\mathrm{K}^{+}$and $\mathrm{Na}^{+}$in the channel and in the aqueous solution (Eisenman, 1962). To address this question, we performed MD FEP, in which $1 \mathrm{~K}^{+}$is alchemically transformed into a Na${ }^{+}$at specific locations along the pore axis (McCammon \& Straatsma, 1992; Kollman, 1993). The calculated free energy, relative to the aqueous solution, is approximately +2.8 and $+6.6 \mathrm{kcal} / \mathrm{mol}$ for site $S_{1}$ and $S_{2}$ respectively. Similar results have been obtained from FEP calculations performed using reduced models of the KcsA channel (Allen et al. 1999, 2000; Aqvist \& Luzhkov, 2000). The selectivity of the so-called 'external lock-in site' detected in $\mathrm{Ba}^{2+}$ blockade experiments (probably site $\mathrm{S}_{2}$; see Jiang \& MacKinnon, 2000) has been estimated to be approximately $+5.5 \mathrm{kcal} / \mathrm{mol}$ (Neyton \& Miller, 1988a, b). In conclusion, the calculations show that the KcsA has the ability to both rapidly conduct $\mathrm{K}^{+}$and discriminate against $\mathrm{Na}^{+}$. However, one should note that the magnitude of the dynamical fluctuations of the carbonyl oxygens forming the selectivity filter is unambiguously larger than the difference in the radius of $\mathrm{Na}^{+}$and $a \mathrm{~K}^{+}$ion, i.e. the atomic fluctuations of the backbone atoms forming the selectivity filter are in the order of $0.75 \AA$, whereas the radii of $\mathrm{K}^{+}$and $\mathrm{Na}^{+}$differ by less than $0 \cdot 4 \AA$ (Roux \& Karplus, 1995; Hille, 2001). Therefore, the high specificity of $\mathrm{KcsA}$ for $\mathrm{K}^{+}$over $\mathrm{Na}^{+}$ions (which is reproduced by the calculations) cannot simply arise from a discrimination based on ion size provided by a rigid pore of a precise geometry. One might think that, in a flexible pore, the selectivity could arise from the slightly greater distortional energy needed to accommodate a smaller cation. However, the 
magnitude of the atomic fluctuations implies that the strain energy associated with sub-angstrom protein distortions is much smaller than $k_{\mathrm{B}} T$. Clearly, further work will be needed to elucidate the microscopic factors that make selectivity a robust property of the $\mathrm{K}^{+}$channel despite thermal fluctuations and protein flexibility.

\subsection{Computational studies of OmpF}

The porins represent ideal systems for addressing fundamental questions about the importance of electrostatic interactions on ion flow through wide aqueous pores. There have been a number of computational studies of porins based on equilibrium MD (Bjorksten et al. 1994; Soares et al. 1995; Watanabe et al. 1997; Suenaga et al. 1998; Tieleman \& Berendsen, 1998; Tieleman et al. 1998; Bond et al. 2002; Faraldo-Gomez et al. 2002, 2003; Im \& Roux, 2002b; Danelon et al. 2003; Zachariae et al. 2003). Several advanced computational techniques have been used to extend the scope of simple equilibrium MD, e.g. MD with applied external electric field (Suenaga et al. 1998; Crozier et al. 2001a, b; Yang et al. 2003), alchemical FEPs (Danelon et al. 2003), and umbrellasampling MD simulations (Zachariae et al. 2003). Nonetheless, the majority of the results aimed at elucidating the role of electrostatic interactions on ion permeation through the porins have been obtained using approximate approaches such as PB (Weiss et al. 1991; Karshikoff et al. 1994; Zachariae et al. 2002), BD (Schirmer \& Phale, 1999; Im et al. 2000; Im \& Roux, 2001, 2002a; Phale et al. 2001), and PNP (Im \& Roux, 2002a).

\subsection{The need to compare the different level of approximations}

Depending on the salt concentration, the permeation process through the wide aqueous pores of $\mathrm{OmpF}$ involves the correlated movements of several ions and counterions; at $1 \mathrm{M} \mathrm{KCl}$, there can be up to $10 \mathrm{~K}^{+}$and $10 \mathrm{Cl}^{-}$in one pore (Im \& Roux, 2002b). But it is not practical to compute all the $n$-ion PMFs describing all the relevant ion configurations needed to represent the multi-ion permeation process using all-atom MD. Furthermore, it is not yet feasible to simulate ion fluxes directly with non-equilibrium MD in the presence of an applied membrane potential (although that should become possible in the near future). Because of these difficulties, a study of ion permeation through $\mathrm{OmpF}$ must rely on a combination of inter-related computational approaches corresponding to different levels of approximation. The different approaches used here are all-atom MD, PB, BD and PNP. How valid are those approaches in the context of ion permeation through $\mathrm{OmpF}$ ? To address this question, we compared the results obtained from all-atom MD, PB, BD and PNP. The atomic system simulated by MD, which comprises the OmpF trimer embedded in a fully solvated phospholipid bilayer membrane bathed by a $1 \mathrm{~m} \mathrm{KCl}$ aqueous salt solution, is shown in Fig. 2c. From a computational point of view, the high salt concentration is advantageous because the large number of ions included explicitly in the simulations significantly helps to obtain statistically meaningful averages of the ion properties in the pore. The results from this all-atom equilibrium MD simulation serve as a benchmark to assess the validity and accuracy of two approaches that are intrinsically more approximate: equilibrium GCMC/BD, in which the solvent is represented as a continuum dielectric but the ions are included explicitly, and $\mathrm{PB}$, in which the both the solvent and the ions are represented as continuum. Accordingly, the PMF underlying the BD/GCMC simulations may be summarized using the acronyms given in Table 1 as PMF(PBV). In the same way, we use the results from nonequilibrium GCMC/BD to assess the validity of PNP. Similar comparison of BD with PB and PNP have been made for pores of simple geometries (Corry et al. 2000; Moy et al. 2000). 


\subsubsection{Equilibrium protein fluctuations and ion distribution}

Overall, the picture emerging from the all-atom $\mathrm{MD}$ simulation of $\mathrm{OmpF}$ in a lipid bilayer suggests that the approximation of a rigid protein and a continuum solvent are qualitatively reasonable, though some aspects of reality are clearly oversimplified by these approximations. The conformation of $\mathrm{OmpF}$ in the membrane is very stable, in large part because of the rigidity of the $\beta$-barrel structure. However, the size of the pore depends most directly on the structural fluctuations of the residues Pro116-Glu117-Phe118-Gly119-Gly120 (PEFGG motif), which forms the most flexible region in the narrowest region of the pore. The orientation of the water molecules in the constriction zone is strongly anisotropic and perpendicular to the pore axis due to the strong transversal electrostatic field arising from the cluster of three arginines (Arg42, Arg82, Arg132) and two acidic residues (Asp113, Glu117) (Cowan et al. 1992; Karshlkoff et al. 1994; Dutzler et al. 1999; Schirmer \& Phale, 1999; Im et al. 2000; Im \& Roux, 2002b). The occurrence of $\mathrm{K}^{+}$and $\mathrm{Cl}^{-}$pairing in the pore is relatively small, though it is more important in the constriction zone, where the pore is narrowest. An ion pair in the constriction zone is shown in Fig. 10c. Permeating ions are not completely dehydrated, which suggest that a continuum dielectric representation of water may be valid. As $\mathrm{K}^{+}$and $\mathrm{Cl}^{-}$move into the $\mathrm{OmpF}$ pore, the contributions from water and the protein atoms varies in a complementary fashion in order to keep the total solvation number of both ions approximately constant. There are approximately four water molecules around both ions in the constriction zone, where their hydration number appears to be minimum. This can be compared with the values in bulk solution, where there are approximately 6.5 and 7.2 water molecules in the first shell of $\mathrm{K}^{+}$and $\mathrm{Cl}^{-}$ions respectively.

To assess the ability of the three approaches, MD, GCMC/BD, and (nonlinear) PB, in describing the equilibrium partitioning of ions in teh pore, we examine the $3 \mathrm{D}$ spatial distribution of $\mathrm{K}^{+}$and $\mathrm{Cl}^{-}$in the $1 \mathrm{~m}$ system. The results are shown in Fig. 20. All three approaches clearly indicate that $\mathrm{K}^{+}$and $\mathrm{Cl}^{-}$ions distribute according to two well-separated average pathways spanning over nearly $40 \AA$ along the axis of the pore. In the center of the monomer, the two screw-like pathways have a left-handed twist, undergoing a counter-clockwise rotation of $180^{\circ}$ from the extracellular vestibule to the pore periplasmic side. Remarkably, GCMC/BD and PB reproduce the well-separated ion pathways, although the screw-like pathway in GCMC/BD and $\mathrm{PB}$ is not as clear as in MD. This seems to suggest that MD samples narrower range of configurations than GCMC/BD or PB. The average number of ions inside the pore are $5.1 \mathrm{~K}^{+}$and 3.9 $\mathrm{Cl}^{-}(\mathrm{MD}), 4.9 \mathrm{~K}^{+}$and $2.8 \mathrm{Cl}^{-}(\mathrm{GCMC} / \mathrm{BD})$, and $5.6 \mathrm{~K}^{+}$and $3.4 \mathrm{Cl}^{-}$(PB) respectively. All three computational approaches show that $\mathrm{K}^{+}$has a higher propensity to occupy the aqueous pore than $\mathrm{Cl}^{-}$, consistent with the cation-selectivity of the OmpF channel. Overall, the agreement is quite reasonable between $\mathrm{MD}$ and $\mathrm{GCMC} / \mathrm{BD}$, indicating that a continuum dielectric description of water with a rigid channel structure in GCMC/BD can be a valid approximation for OmpF porin. Furthermore, the present analysis shows that the (nonlinear) PB is able to capture the important electrostatic interactions between ions in the aqueous pore.

\subsubsection{Non-equilibrium ion fluxes}

On the basis of the macroscopic helical paths shown in Fig. 20, one can envision the average journey of $\mathrm{K}^{+}$and $\mathrm{Cl}^{-}$ions from the extracellular side to the periplasmic side. But, in order to fully characterize $\mathrm{OmpF}$, it is necessary to simulate non-equilibrium ion flow. This is done with GCMC/BD or with PNP. The transmembrane potential $\phi_{\mathrm{mp}}(\mathbf{r})$, which is needed to generate 

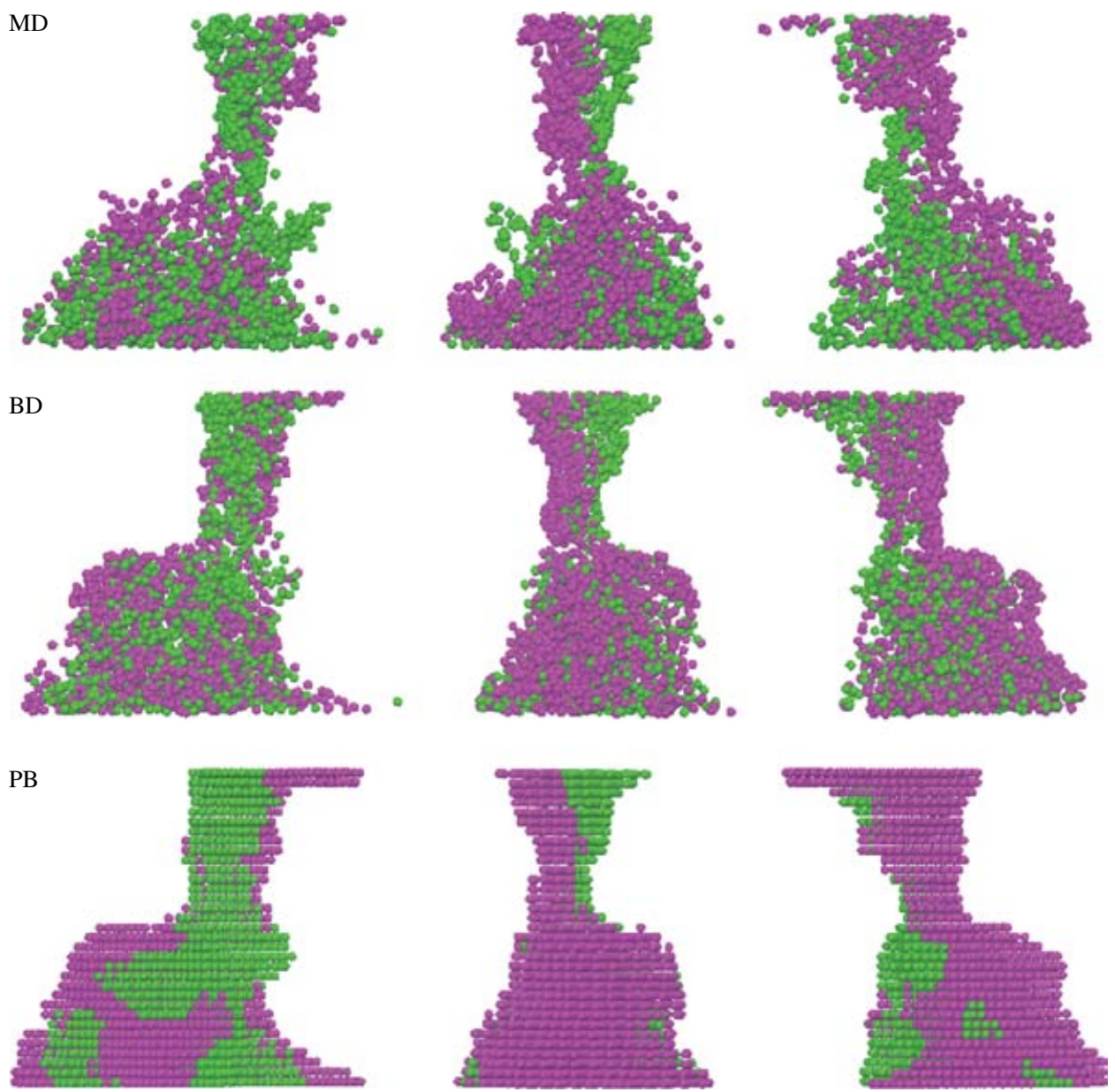

Fig. 20. Two well-separated specific ion pathways with a left-handed screw-like fashion. The $\mathrm{K}^{+}$ions are magenta and the $\mathrm{Cl}^{-}$ions are green. MD: A superimposition of 100 snapshots of ions every 50 ps from the 5 ns trajectory; all the ions in two other pores were superimposed into one pore by rotations. BD: A superimposition of 300 snapshots of ions every 60 ps from the 60 ns trajectory. PB: A ion distribution 3D grid map. Left: view from perpendicular to the three-fold symmetric axis. Middle: left view rotated by $120^{\circ}$. Right: left view rotated by $240^{\circ}$. The figure was produced with DINO (http://www.dino3d.org).

non-equilibrium GCMC/BD simulations, is readily incorporated into the static field via PB-V Eq. (125). The potential profile along the axis of the pore is illustrated in Fig. 21. It is observed that the dominant effect of the transmembrane potential is in the narrowest part of the pore, where the driving field is approximately linear. The profiles of the diffusion coefficients for $\mathrm{K}^{+}$and $\mathrm{Cl}^{-}$along the pore is also the important ingredient needed for GCMC/BD and PNP. The profiles of the diffusion coefficient of $\mathrm{K}^{+}$and $\mathrm{Cl}^{-}$along the channel axis, calculated using Eq. (107) from the all-atom MD simulation of $\mathrm{OmpF}$ at $1 \mathrm{M} \mathrm{KCl}$, are shown in Fig. 22. It is observed that, inside the pore, the diffusion coefficient of $\mathrm{K}^{+}$and $\mathrm{Cl}^{-}$is reduced to $\sim 50 \%$ relative to their value in bulk solution. The lack of abrupt variations in the diffusion coefficient suggests that the random walk of the ions inside $\mathrm{OmpF}$ is largely governed by the structure of the multi-ion PMF rather than by the dissipative factors themselves. 


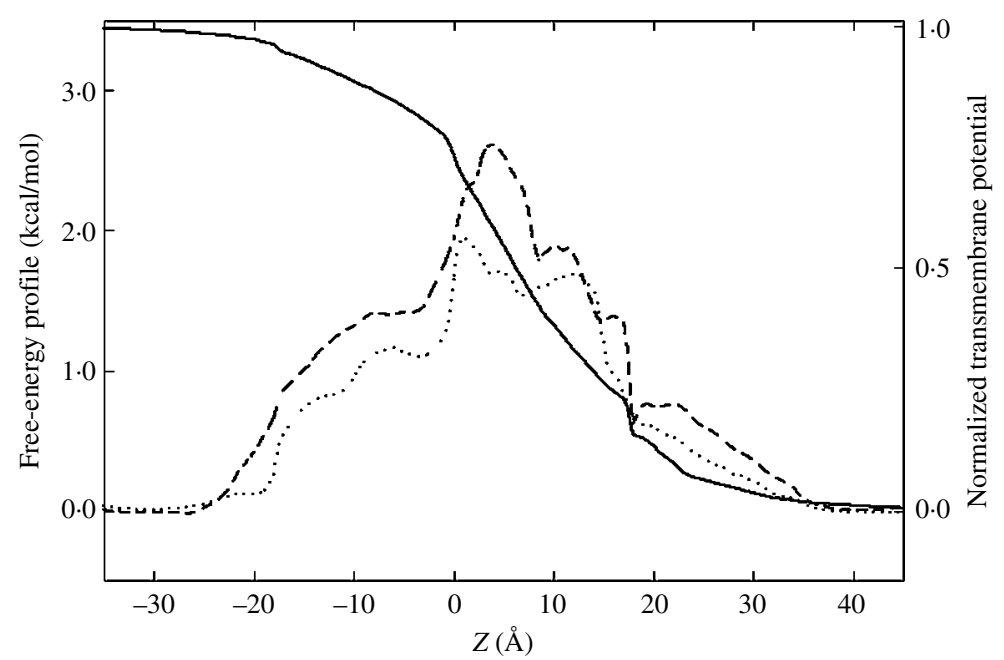

Fig. 21. One-dimensional free-energy profiles of $\mathrm{K}^{+}$(dotted line) and $\mathrm{Cl}^{-}$(dashed line) with the transmembrane potential of $+150 \mathrm{mV}$ (solid line). $150 \mathrm{mV}$ corresponds to $3.46 \mathrm{kcal} /(\mathrm{mol} \cdot e$ ). The fraction of the transmembrane potential is also shown on the right. The free-energy profiles of both ions were calculated as $\mathcal{W}_{\alpha}(z)=-k_{\mathrm{B}} T \ln \left(\rho_{\alpha}(\mathrm{z}) / \rho_{\alpha}^{\text {bulk }}\right)$ in a $1 \mathrm{M} \mathrm{KCl}$ symmetric solution, where $\rho_{\alpha}(\mathrm{z})$ was calculated using a $0.5 \AA$ bin along the $z$-axis and the cross-sectional area of the GCMC/BD system in the $x y$-plane.

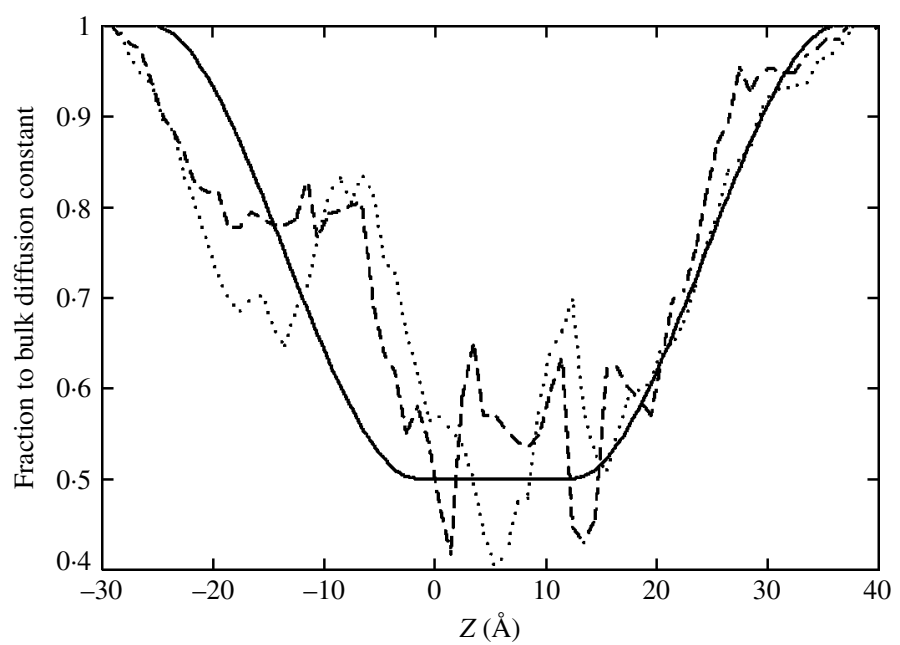

Fig. 22. A (fractional) non-uniform diffusion coefficient profile for $\mathrm{K}^{+}$and $\mathrm{Cl}^{-}$ions in GCMC/BD and PNP; 1 when $z>36.5$ or $z<-25.5,0.5\left(f_{\min }\right)$ when $-1.5<z<12.5$, and $f_{\min }+\left(1-f_{\min }\right) * g_{\text {sw }}$ in switching regions, where a switching function centered at $z_{c}=5.5$ over $L=24 \AA$ length, $g_{\text {sw }}=$ $\pm 2 *\left(\left(z-z_{c}\right) / L\right)^{3}-3 *\left(\left(z-z_{c}\right) / L\right)^{2}$, is applied. For comparison, the molecular dynamics results for $\mathrm{K}^{+}$ (dotted line) and $\mathrm{Cl}^{-}$(dashed line) scaled by their bulk values are also shown. We used $0.196\left(\mathrm{~K}^{+}\right)$and $0 \cdot 203\left(\mathrm{Cl}^{-}\right) \AA^{2} / \mathrm{ps}$ for the bulk diffusion coefficient (Hille, 2001).

The calculated current-voltage $(I-V)$ relation in $1 \mathrm{M} \mathrm{KCl}$ is shown in Fig. 23. The $I-V$ relation appears to be asymmetric, i.e. both $I_{\mathrm{K}}$ and $I_{\mathrm{Cl}}$ at a positive potential are always larger than those at the corresponding negative potential, resulting in the asymmetry in the channel conductance upon the polarity of the applied potential. At $1 \mathrm{~m} \mathrm{KCl}$, the conductance of $\mathrm{OmpF}$ 

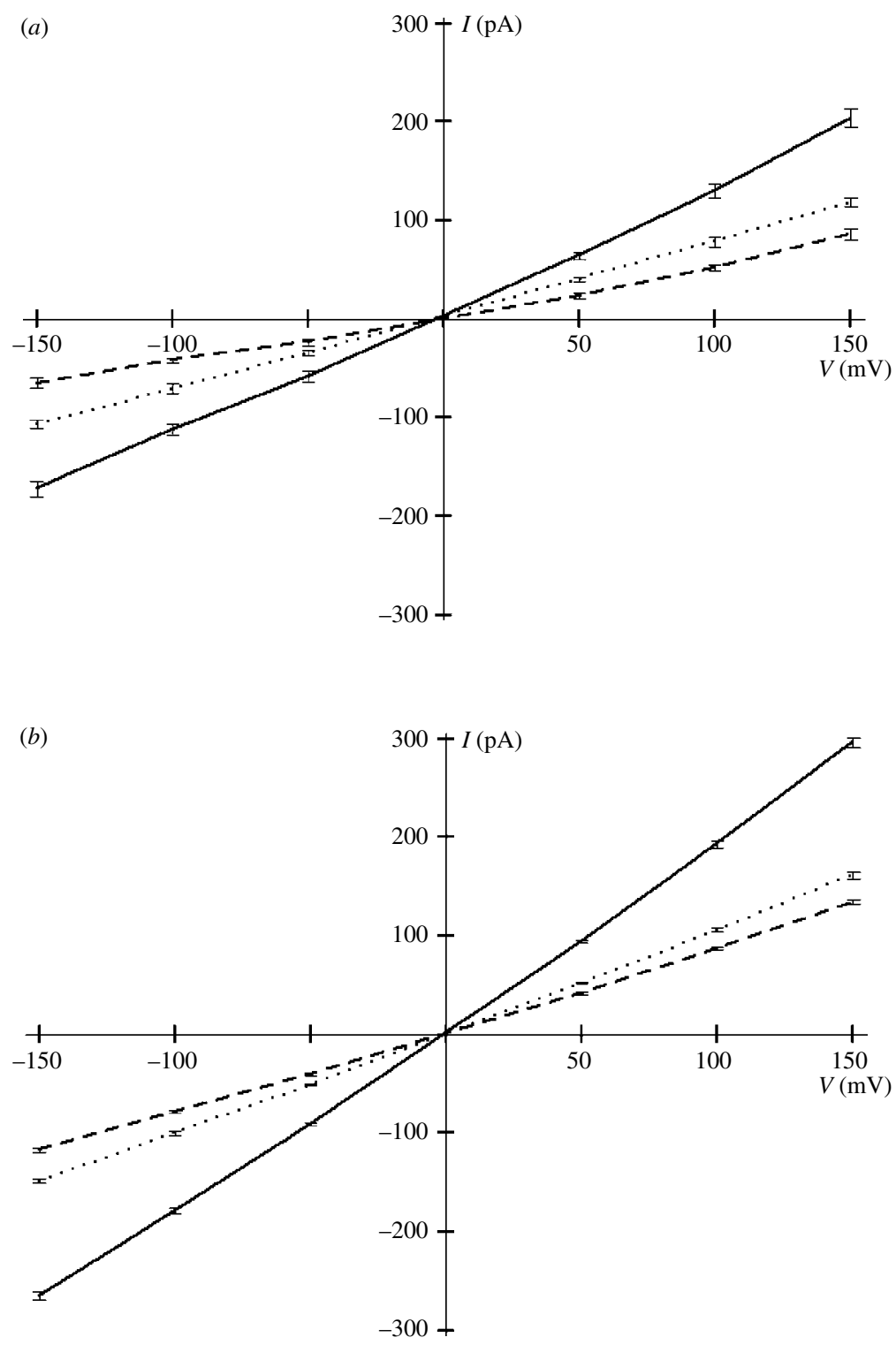

Fig. 23. Current-voltage curve in a $1 \mathrm{M} \mathrm{KCl} \mathrm{symmetric} \mathrm{solution} \mathrm{from} \mathrm{(a)} \mathrm{GCMC/BD} \mathrm{simulations} \mathrm{and} \mathrm{(b)}$ PNP calculations. The total current (solid line) is the sum of $\mathrm{K}^{+}$(dotted line) and $\mathrm{Cl}^{-}$(dashed line) currents.

calculated with GCMC/BD is 1.36 and $1.15 \mathrm{nS}$ for $\pm 150 \mathrm{mV}$ respectively. Under the same condition, the conductance calculated with PNP is 1.97 and $1.77 \mathrm{nS}$ for $\pm 150 \mathrm{mV}$ respectively. For comparison, the measured conductance of $\mathrm{OmpF}$ inserted in a planar lipid bilayer with the same salt concentration is 1.25 and $1.13 \mathrm{nS}$ at $\pm 160 \mathrm{mV}$ respectively (N. Saint, personal communication). Although the conductance and its asymmetry calculated from GCMC/BD is slightly higher than the experimental data, the agreement is excellent (no parameters were specifically adjusted to match those experimental data). The ion-accessible aqueous pore of $\mathrm{OmpF}$ is markedly asymmetric, with a relatively small extracellular vestibule and a large 


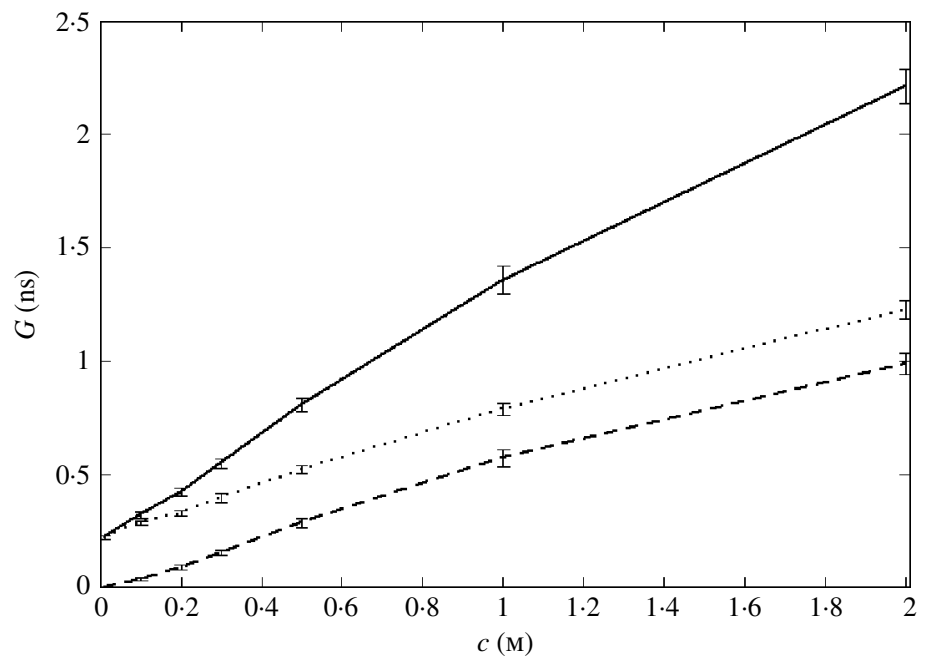

Fig. 24. Conductance-concentration (G-c) relation (solid line) from GCMC/BD at $V_{\mathrm{mp}}=+150 \mathrm{mV}$. The contributions from $\mathrm{K}^{+}$(dotted line) and $\mathrm{Cl}^{-}$(dashed line) ions are also shown. Similar results were obtained from $\mathrm{PB}$ or PNP, but are not shown for clarity.

intracellular vestibule. The asymmetry increases with decreasing salt concentration, indicating that the permanent charge distribution of $\mathrm{OmpF}$ porin is responsible rather than the irregular geometry of the pore itself.

The conductance-concentration relation, shown in Fig. 24, appears to be sublinear at high concentrations. There are $1.55 \mathrm{~K}^{+}$inside the pore in $0.01 \mathrm{M}$, which is only about three times smaller than the number of $\mathrm{K}^{+}$in $1 \mathrm{M} \mathrm{KCl}$, whereas the number of $\mathrm{Cl}^{-}$ions is then almost negligible (additional simulations indicate that there is still roughly $0.17 \mathrm{~K}^{+}$on average inside the pore even in $1 \mu \mathrm{M})$. As a result, the conductance at low concentration is significantly larger than a simple naive extrapolation from the value at high concentration would suggest. Qualitatively similar results are obtained with PNP.

\subsubsection{Reversal potential and selectivity}

According to both non-equilibrium GCMC/BD and PNP calculations, more current is carried by $\mathrm{K}^{+}$ions than $\mathrm{Cl}^{-}$ions. This is consistent with the cation selectivity of the channel (Saint $e t$ al. 1996). Assuming that the current ratio $I_{\mathrm{K}} / I_{\mathrm{Cl}}$ is representative of the channel selectivity at a given concentration, $\mathrm{OmpF}$ becomes increasingly more selective for $\mathrm{K}^{+}$and excludes $\mathrm{Cl}^{-}$as the concentration is decreased because of the reduction of ionic screening of the negative electrostatic potential arising from the protein charges. Experimentally, the selectivity of porins is extracted from a measurement of the reversal potential at zero net current in asymmetric salt solution. The permeability ratio is then obtained from the Goldman-Hodgkin-Katz (GHK) voltage equation (Goldman, 1943; Hodgkin \& Katz, 1949; Hille, 2001).

$$
V_{\mathrm{rev}}=\frac{k_{\mathrm{B}} T}{e} \ln \left[\frac{P_{\mathrm{K}}[C]_{\mathrm{o}}+P_{\mathrm{CI}}[C]_{\mathrm{i}}}{P_{\mathrm{K}}[C]_{\mathrm{i}}+P_{\mathrm{Cl}}[C]_{\mathrm{o}}}\right] \text {, }
$$

where $[C]_{\mathrm{i}}$ and $[C]_{\mathrm{o}}$ are the intracellular and extracellular $\mathrm{KCl}$ concentrations. Therefore, in order to rigorously examine the charge specificity of $\mathrm{OmpF}$, it is necessary to simulate the 

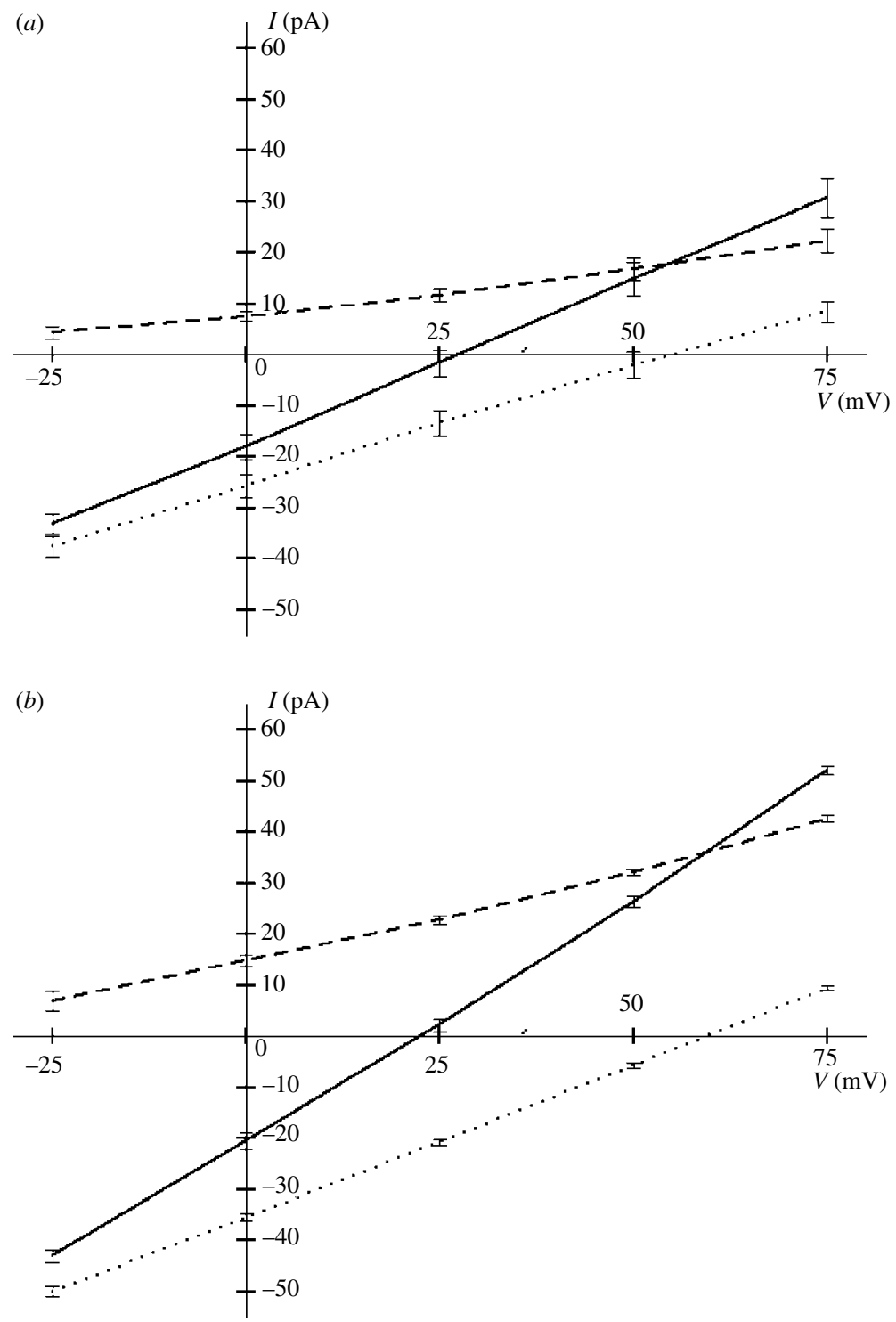

Fig. 25. Current-voltage curve in $0 \cdot 1: 1 \mathrm{M} \mathrm{KCl}$ asymmetric solution from (a) GCMC/BD simulations and (b) PNP calculations. The total current (solid line) is the sum of $\mathrm{K}^{+}$(dotted line) and $\mathrm{Cl}^{-}$(dashed line) currents.

correct non-equilibrium conditions that are used in experiments. Figure 25 shows the $I-V$ curves calculated from GCMC/BD and PNP in the case of asymmetric salt solution of $0 \cdot 1: 1 \mathrm{M} \mathrm{KCl}$. The reversal potential, $V_{\text {rev }}$, at zero net current is determined to be $27.4 \mathrm{mV}$ (GCMC/BD) and $22.1 \mathrm{mV}$ (PNP), in excellent accord with the experimental value of $24.3 \mathrm{mV}$ (Schirmer \& Phale, 1999).

The reversal potentials calculated from PNP are reasonable but the ion current is overestimated by $\sim 40-50 \%$ relative to GCMC/BD. Empirically scaling down the diffusion coefficient of the ions inside the pore produces a similar $I-V$ relation to GCMC/BD from PNP calculations. Such a scaling of the diffusion coefficient of both $\mathrm{K}^{+}$and $\mathrm{Cl}^{-}$changes only the 
slope of the $I-V$ curve but does not affect the value of the reversal potential. This suggests that the PNP mean-field approximation can be helpful to understand the dominant features of ion permeation and selectivity through $\mathrm{OmpF}$. Interestingly, the $I-V$ curves calculated from both GCMC/BD and PNP show that the current carried by each ionic species becomes nearly zero at its own Nernst equilibrium potential, as if the flux of one type of ion is not strongly affected by the flux of other ions. The value of the reversal potential from PNP follows directly from the sum of two currents of different magnitude that behave independently from one another. The value of the voltage at which the total current becomes zero is more or less determined by the relative magnitude of the two currents. In this context, one might anticipate that the ratio of the currents at zero potential under asymmetric conditions of concentration $\left|I_{\mathrm{K}} / I_{\mathrm{Cl}}\right|$ should be closely related to the ratio of the permeability coefficients $P_{\mathrm{K}} / P_{\mathrm{Cl}}$. The permeability ratios extracted from the GHK voltage equation are 3.9 (BD) and 2.9 (PNP). Remarkably, the calculated current ratios at zero potential are 3.4 (BD) and 2.4 (PNP), in excellent accord with the corresponding permeability ratios.

The apparent applicability of the GHK voltage equation in the context of a complex molecular pore is intriguing and surprising. The GHK equation is derived using a 1D-NP equation under the simplifying assumptions that the potential is linear over the length of the pore and that the diffusion coefficient is constant throughout the pore (Goldman, 1943; Hodgkin \& Katz, 1949; Hille, 2001). Those assumptions are not satisfied in the case of OmpF. Furthermore, the presence of $\mathrm{K}^{+}$ions in the $\mathrm{OmpF}$ pore is needed to screen the excess negative electrostatic potential arising from $\mathrm{OmpF}$ and help permeation of $\mathrm{Cl}^{-}$ions. This observation represents a clear violation of ion independence, which is an essential assumption in deriving the GHK equations (Goldman, 1943; Hodgkin \& Katz, 1949; Hille, 2001). However, within the framework of the GHK voltage equation, it can be shown that the equivalence between the current and permeability ratios is preserved if the free-energy barriers opposing the passage of cations and anions are located along the channel axis at a position where the transmembrane potential is roughly half of $V_{\mathrm{mp}}$ (Im \& Roux, 2002a). Average 'effective' 1D freeenergy profiles of $\mathrm{K}^{+}$and $\mathrm{Cl}^{-}$along the pore axis can be calculated from the average equilibrium density of ions,

$\mathcal{W}_{\alpha}(z)=-k_{\mathrm{B}} T \ln \left(\frac{\left\langle\rho_{\alpha}(z)\right\rangle}{\bar{\rho}_{\alpha}}\right)$

As shown in Fig. 21, the barrier in the effective free-energy profile is located where the transmembrane potential is approximately half of $V_{\mathrm{mp}}$. This is the reason why, despite the presence of strong ion-ion correlations, the system behaves as if there is some effective independence of the ion fluxes.

Based on a comparison of PB and PNP with BD in various simple model pores, Chung and co-workers argued that there should be large discrepancies between BD and so-called mean-field theories such as PB or PNP because the latter two methods overestimate the shielding effect by counterions due to underestimation of the self-energy in narrow channels (Moy et al. 2000; Corry et al. 2000). Such a large discrepancy between the two theories is not observed in our simulations of $\mathrm{OmpF}$, presumably because the pore is sufficiently wide and the electrostatic potential arising from the fixed charges of the protein atoms is quite strong. Interestingly, although $\mathrm{BD}$ and $\mathrm{PB}$ are in excellent accord for equilibrium situations, the ionic currents calculated from PNP are always larger than those from BD simulations. Since all the microscopic 
model and input parameters are identical in the two models, the difference between BD and PNP is necessarily caused by the mean-field approximation in which some dynamical ion-ion correlations are neglected. This is consistent with the fact that a significant amount of ion-ion pairing was observed in the narrowest region of the OmpF pore during MD simulation (Im \& Roux, 2002b). In bulk solution, it is known that such correlations which increase with ion concentration result in a small reduction of the effective ion diffusion coefficient (by a few per cent) (Mills \& Lobo, 1989). Correlations are particularly important here because the ion concentration inside the molecular pore is much higher than the bulk concentration, e.g. in $1 \mathrm{M} \mathrm{KCl}$ solution both $\mathrm{K}^{+}$and $\mathrm{Cl}^{-}$concentrations in the constric zone of $\mathrm{OmpF}$ are more than $5 \mathrm{~m}$. Thus, a reduction of ion mobility which is not captured in PNP is expected inside the pore.

GCMC/BD multi-ion simulations, constructed upon the information extracted from all-atom $\mathrm{MD}$, is a powerful approach to examine non-equilibrium ion flow in wide aqueous pores such as $\mathrm{OmpF}$ and address questions about the microscopic origin of their charge specificity. The existence of two well-separated pathways for cations and anions suggests that the charge specificity of $\mathrm{OmpF}$ porin does not arise from a few local interactions in the constriction zone, but rather from a number of residue distributed over a large fraction of the aqueous pore (Im \& Roux, 2002a, b). Future efforts will focus on quantitatively characterizing the effect of site-directed mutuations on the conductance and cation/anion selectivity for OmpF, PhoE and OmpK36, and comparing with available experimental data.

\subsection{Successes and limitations}

\subsection{Channel structure}

In trying to assess the strengths and weaknesses of the various computational models, it is important to clearly distinguish the equilibrium and the non-equilibrium aspects. As emphasized previously in sections $2-4$, an accurate representation of equilibrium properties is essential for meaningful studies of ion permeation. In particular, the overall stability and integrity of the channel structure during the dynamical trajectory is one important indicator of the validity of all-atom MD simulations. It should be emphasized that accurate experimental structures, determined at atomic resolution, are absolutely essential to carry detailed theoretical studies of ion permeation. Atomic resolution X-ray crystallographic structures were available for both KcsA and OmpF. During the MD trajectories of those channels, the protein conformation typically was very stable, with relatively small deviations from the experimental structures. In the case of gA, the available structures were determined by solution (Townsley et al. 2001) and solid-state NMR (Ketchem et al. 1997). The two structures are similar but display some differences and assessing their relative accuracy is difficult. Nonetheless, dynamical averages taken from the channel simulated in a membrane were shown to be in excellent agreement with the solid-state NMR experimental data (Woolf \& Roux, 1994b; Allen et al. 2003a), demonstrating that the overall accuracy of the current atomic model of gA.

\subsection{2 lon-binding sites}

A correct representation of the energetically favorable locations along the permeation pathway (binding sites) is of paramount importance to begin understanding the selectivity and the conduction properties of narrow biological channels. In the case of $\mathrm{gA}$, cation-binding sites are 
found around 9-11 $\mathrm{A}$, at each ends of the channel, in good agreement with experiments. However, the precise location of the cation-binding site appears to be very sensitive to the details of the potential function, with small variations of $1 \cdot 0-1 \cdot 5 \AA$. According to various experimental measurements, the actual position of the main binding site is probably close to $9.5 \AA$. Many of the previous calculations based on atomic models have found cation-binding sites at 9.5 and $11 \AA$, near the entrance of the gA channel. In the case of KcsA, the original X-ray structure provided only a few elements of information about the binding sites of $\mathrm{K}^{+}$in the selectivity filter (Doyle et al. 1998). The PMF calculation reproduced all the main four cation-binding sites located in the narrow selectivity filter (Doyle et al. 1998; Zhou et al. 2001b), though the position of site $\mathrm{S}_{0}$ differs by $\sim 1.5 \AA$ from the high-resolution structure. There is no direct information about the position of bound ions in the $\mathrm{X}$-ray structure of $\mathrm{OmpF}$, therefore, only a comparison of the computational results from MD, BD/GCMC and PB is possible. Remarkably, in all three approaches, $\mathrm{K}^{+}$and $\mathrm{Cl}^{-}$ions follow two well-separated left-handed screw-like average pathways undergoing a counter-clockwise rotation of $180^{\circ}$ from the extracellular vestibule to the pore periplasmic side that spans over $40 \AA$ along the axis of the pore. This shows that the approximate models $\mathrm{BD} / \mathrm{GCMC}$ and $\mathrm{PB}$ are able to capture the most important features of the electrostatic interactions between ions and the charge distribution of OmpF. The existence of two wellseparated pathways for cations and anions suggests that the charge specificity of OmpF porin does not arise from a few local interactions in the constriction zone, but rather from a number of residues distributed over a large fraction of the aqueous pore (Im \& Roux, 2002b). The results for the narrow channels gA and KcsA show that MD simulations based on carefully constructed atomic models are able to describe the location of the main ion binding sites with an accuracy of $\sim 1.0-1.5 \AA$. In contrast, models that use a rigid channel structure and a continuum solvent approximation are unable to reproduce this essential feature of those channels (Edwards et al. 2002). In the case of gA, a PNP calculation found the dominant binding site in the center of the dimer, nearly $10 \AA$ away from the correct position (Kurnikova et al. 1999). In the case of $\mathrm{KcsA}$, BD simulations do not reproduce any of the five most important $\mathrm{K}^{+}$-binding sites $\left(\mathrm{S}_{0}-\mathrm{S}_{4}\right)$ located in the selectivity filter (Mashl et al. 2001; Chung et al. 2002; Burykin et al. 2002, 2003). This in itself shows that simplified models, which treat the protein rigidly and represent the solvent as a dielectric continuum, are severely limited. Although such approximations provide a qualitative representation of a multi-ion channel, they cannot capture the influence of the structural dynamical fluctuations of the selectivity filter observed in MD (Guidoni et al. 1999; Allen et al. 2000; Åqvist \& Luzhkov, 2000; Bernèche \& Roux, 2000, 2001; Shrivastava \& Sansom, 2000).

\subsubsection{Ion conduction}

The ion conductance is by far the most difficult property of an ion channel to calculate with quantitative accuracy from an all-atom model. A small deviation in the equilibrium PMF of permeating ion is sufficient to change the ion flux by several orders of magnitude. Furthermore, the absolute conductance is generally more difficult to calculate because it is very sensitive to the all details of the environment of the channel, the buffer solution, and the lipid composition. In the case of $\mathrm{gA}$ and $\mathrm{KcsA}$, modern all-atom potential functions together with a computational strategy based on a systematic sampling of the PMF has allowed the conductance to be computed with reasonable success. For gA, the maximum conductance appears to be underestimated by 20 - to 30 -fold. In contrast, the calculated maximum conductance of the selectivity filter 
of $\mathrm{KcsA}$ is in remarkable agreement with experimental data. Because a small increase of about $k_{\mathrm{B}} T$ in one energy barrier along the ion conduction pathway would be sufficient to decrease the ion flux by a factor of almost 3, it is clear that such a striking success is partly fortuitous. Nonetheless, considering that none of the microscopic parameters used here were optimized specifically for these systems, the present results are very encouraging. In the case of OmpF, a multi-ion continuum electrostatic GCMC/BD approach was used and the conductance was reproduced over a very wide range of concentration, in excellent agreement with experiments. These results show that a simplified model in which the protein channel is represented as a rigid structure and the solvent as a continuum dielectric is reasonable to simulate ion permeation through a wide aqueous pore such as $\mathrm{OmpF}$. These approximations are valid in this case because the ions are not dehydrated and are not tightly coordinated by the protein they are translocated through the pore; it may be all right to ignore the $0 \cdot 5-1 \cdot 0 \AA$ RMS atomic fluctuations of the protein in the case of a pore of $6 \AA$ diameter.

Although gA is the simplest channel considered here, the calculated conductance displays the largest discrepancy with the experimentally value. Why are the results from MD apparently better for KcsA than for gA since both are narrow pores lined by backbone carbonyls? In the case of $\mathrm{gA}$, two corrections were introduced in the PMF: for the spurious destabilization arising from the finite-size and periodicity of the simulated system $(-1.6 \mathrm{kcal} / \mathrm{mol})$, and the neglect of induced polarization of the lipid hydrocarbon chains $(-2 \cdot 1 \mathrm{kcal} / \mathrm{mol})$. An estimate of the same corrections reveals that they would be much smaller in the case of KcsA. The finite-size periodicity effect is negligible because the size of the periodic box used for MD is much larger, the polarization of the hydrocarbon is also negligible because the lipid chains are much further away. Some additional energetic and structural effects are perhaps also at play. In gA, $\beta$-helical backbone hydrogen bonds involving the carbonyl groups must be slightly perturbed to stabilize the permeating cation; some amount of carbonyl reorientation is absolutely required for stabilizing the cation (Chiu et al. 1993; Dorman et al. 1996; Woolf \& Roux, 1997). Modeling this process requires a very accurate representation of not only intermolecular ion-channel and ionwater interactions, but also intramolecular channel-channel interactions. Clearly, such delicate balance of interactions (ion-peptide versus peptide-peptide) is imperfectly represented by current non-polarizable force fields. In contrast, the structure of the selectivity filter of the KcsA, which evolved for maximum throughput, can conduct $\mathrm{K}^{+}$without altering its structure appreciably. For instance, the carbonyl group point directly toward the center of the pore. For this reason, small inaccuracies in channel-channel interactions do not have as large an impact as in the case of gA. Nevertheless, the present results demonstrate that the calculation of the permeation properties of a selective ion channel with semi-quantitative accuracy, from first principles, is possible.

\subsubsection{Ion selectivity}

The selectivity is a very important property of ion channels which can be addressed by computational models. As discussed in Section 4.4, FEP calculations based on MD simulations are an appropriate strategy to examine the selectivity of narrow selective pore. For example, MD simulations and FEP calculations revealed the microscopic basis of the cation specificity of the gA channel (Roux, 1996). In the case of a wide multi-ion pore such as OmpF, which exhibits only a moderate charge-specific selectivity, it becomes more difficult to use FEP calculations because the number of relevant states of occupancy becomes unwieldy. Such a case is better described in terms of the reversal potential established at steady-state in the presence of 
asymmetric ionic solutions. The selectivity of $\mathrm{KcsA}$ for $\mathrm{K}^{+}$against $\mathrm{Na}^{+}$is a much more difficult problem, because it concerns two cations of very similar size. Remarkably, in this case as well, a number of FEP calculations based on different simulation methodologies are in general agreement with experimental data (Allen et al. 1999, 2000; Åqvist \& Luzhkov, 2000; Bernèche \& Roux, 2001; Luzhkov \& Aqvist, 2001a). This suggest that the dominant factors giving rise to the selectivity of $\mathrm{K}^{+}$channels is incorporated, at least in an approximate way, in the current models. Nonetheless, it is likely that induced polarization will play an important role and that current biomolecular force field can provide only a semi-quantitative representation of the interaction between small metal cations and the selectivity filter (Roux, 1993; Guidoni \& Carloni, 2002). Lastly, it should be stressed that questions about the ion selectivity in narrow channels only can be addressed in a meaningful way using all-atom MD models that allow the full flexibility of the channel. Models that treat the protein rigidly and represent the solvent as a dielectric continuum are not able to account for the exquisite ion selectivity observed in narrow channels because sub-angstrom atomic displacements of the channel can have a huge energetic impact.

\section{Conclusion}

In this review, we have developed a theoretical and computational framework based on a rigorous formulation of a hierarchy of dynamical models to describe ion permeation. As illustrated by the results discussed in Section 6, adopting this strategy helped considerably in making important progress in understanding $\mathrm{gA}, \mathrm{KcsA}$ and $\mathrm{OmpF}$. Comparison with experiments demonstrates that current computational models are rapidly approaching semi-quantitative accuracy. But beyond the specifics of the actual numerical results, we would like to emphasize that an important strength of the present strategy is to help provide a rigorous conceptual framework to discuss ion permeation rationally. Theoretical models, at any level, are approximations. While classical trajectories are generated using an approximate potential function in all-atom MD simulations, PB, PNP and BD representing the solvent as a continuum dielectric are based on further simplifications which leave out some of the atomic reality we know is important at the microscopic level (e.g. van der Waals interactions, core-core repulsion, hydrogen bonding, induced polarizability, hydration structure, and protein flexibility). Simplified models can still be very valuable when they assist in highlighting some fundamental principles in a particularly clear fashion. For example, Eisenman (1962) was able to use exceedingly simple arguments based on ion size to provide a very insightful perspective on selectivity; Parsegian (1969), Levitt (1978) and Jordan (1981) used calculations based on continuum electrostatic models to reveal important principles of ion permeation through membrane channels; a PNP mean-field treatment in one-dimension provides an incisive perspective on the importance of electrostatics in the selectivity of calcium channels for divalent and monovalent ions (Nonner et al. 2000); semi-microscopic models including a few discrete water molecules have clarified important energetic principles about ion solvation in narrow pores (Dorman et al. 1996; Garofoli \& Jordan, 2003); many fundamental aspects of single file diffusion in multi-ion pore were revealed by studies based on the rate models of Hille \& Schwarz (1978), BD in one-dimension (Bek \& Jakobsson, 1994) and three-dimensions (Allen \& Chung, 2001). In the last few years, the development of robust and stable finite-difference algorithms to solve the PNP equations (Kurnikova et al. 1999) as well as detailed multi-ion BD models in 3D space with a consistent treatment of continuum electrostatic static and reaction fields (Chung et al. 1998) made it possible 
to test explicitly the validity of PNP mean-field electrodiffusion theories for channel models of arbitrary geometries (Corry et al. 2000; Moy et al. 2000; Im \& Roux, 2002a).

But simple models must also be used wisely, within the limits of their validity. As a principle, all theoretical approaches used to describe ion permeation should be, as much as possible, solidly anchored into the physical reality of these macromolecular systems. The properties of the physical system being investigated dictate which approximations are suitable for a given problem; it is not a matter of personal preference. Ultimately, the significance of approximate models should not be expected to exceed the validity of the approximations upon which they are built and approaches that do not explicitly acknowledge this may be seriously in error. For example, most approaches related to continuum electrostatics need to map out the atomic charge distribution as well as the space-dependent dielectric constant onto a numerical grid for each calculation of the electrostatic potential. In those calculations, the channel is generally represented as a static rigid structure and the influence of atomic fluctuations is ignored. This is a drastic approximation which is incorrect in the case of narrow channels where the small atomic fluctuations of the protein can have a very large energetic impact. The importance of dynamical fluctuations and the structure of the water molecules in single file in a narrow pore were revealed by the MD simulations of the gA channel performed by Mackay et al. (1984). Yet, it seems that even 20 years later many of these observations need to be restated again. Atomic fluctuations of proteins are usually in the order of $0 \cdot 5-1 \cdot 0 \AA$ RMS. Ion-protein interactions are very large and the flexibility of ion channels, as of any proteins, plays an important role in its function. Furthermore, the validity of approximate treatments that represent the solvent as a structureless dielectric continuum is probably quite limited, particularly in the case of narrow pores. Although continuum electrostatics is successful in treating processes taking place in bulk solution, there are significant effects arising from the granularity of water molecules and their ability to form hydrogen bonds. In bulk solution, continuum dielectric behavior is observed only at distances larger than a few water diameters, i.e. 5-6 A (Pettitt \& Rossky, 1986); the effective ion-ion interaction energy has some microsocpic structure (wells and barriers) and deviates from the smooth and simple Coulomb's law, $q_{1} q_{2} / \varepsilon r_{12}$. For example, the PMF between two anions or two cations in bulk water are different, whereas continuum electrostatics is unable to make that distinction. In single-file channels the deviations from the continuum behavior are expected to be even more significant. For example, cation-cation interactions in the gA channel are speciesdependent even at a distance of $20 \AA$ because of the specific interactions with water molecules (Becker et al. 1992; Roux et al. 1995).

Representations that are more detailed and closer to the physical reality should serve as benchmarks to assess the validity of more approximate models. Even atomic models and potential functions used in MD simulations may correspond to varying degrees of accuracy. Some potential function include all atoms, e.g. AMBER (Cornell et al. 1995) and CHARMM PARAM22 (Schlenkrich et al. 1996; MacKerell et al. 1998), whereas others are extended-atom models which treat only the polar hydrogens (those able to form hydrogen bonds) explicitly, e.g. CHARMM PARAM19 (Brooks et al. 1983) and GROMOS (van Gunsteren et al. 1999). Furthermore, the treatment of electrostatic interactions, with or without truncation, has major implications for the significance of the results obtained by MD. Unavoidably, these differences lead to considerable variations in the accuracy and significance of MD simulations that are generated (Roux \& Bernèche, 2002). One direction where important progress is eagerly anticipated is in the development of a biomolecular force field including the explicit treatment of induced polarizability. Current biomolecular potential functions typically account for many-body 
polarization effects in an average way using an effective parametrization of the atomic partial charges (Becker et al. 2001). Because of this approximation, the optimal parametrization is the result of a compromise between an accurate representation of the microscopic energies and bulk solvation properties. Such potential functions can yield meaningful results of semi-quantitative accuracy, as long as they are based on effective potential functions that have been calibrated to correctly reproduce solvation free energies (Roux \& Bernèche, 2002). But such an effective force field may not be consistently reliable, under all the conditions relevant to biomolecules. For example, this is one reason why it is possible to obtain excellent agreement with experiments for KcsA while there are additional difficulties with gA (see sections 6.1, 6.2). If the potential function was an exact representation of the Born-Oppenheimer energy surface, success in reproducing the microscopic interactions would automatically lead to accurate thermodynamic properties (assuming quantum effects involving the nuclei are negligible). Accordingly, improvements in the representation of biomolecules are anticipated if non-additive manybody polarization is explicitly taken into account. Currently, the development of a new generation of force fields for computational studies of biological systems that will include induced polarization is actively pursued (Rick et al. 1994; Halgren \& Damm, 2001 ; Lamoureux et al. 2003). Lastly, a very powerful approach is to calculate the Born-Oppenheimer energy surface 'onthe-fly' by considering explicitly the electronic structure at the quantum mechanical level using density functional theory (DFT) (Sagnella et al. 1996; Guidoni \& Carloni, 2002). But this approach is computationally intensive, which severely limits the length of the trajectories that can be generated (typically 10-20 ps), the amount of sampling that can be achieved, and the possibility to make contact with experiments.

Our own perspective on ion permeation has been developed in the context of atomic models from the statistical mechanical Mori-Zwanzig projection-operator formalism (Zwanzig, 2001). Within this framework, the fundamental key ingredients such as the PMF and the memory function (related to the friction coefficient) are best calculated using all-atom detailed MD models. Many of these ideas were first evoked by Kent Wilson during the Biophysical Discussion on Ionic Channels in Membranes in 1983 (Airlie House, Virginia). As illustrated in Section 6, such a hierarchy of inter-related ion permeation models provides a powerful approach to set up and design practical computational strategies for calculating ion fluxes. It is extremely satisfying that many of the results are qualitatively coherent with classical theoretical models of ion permeation widely used by electrophysiologists (see Hille, 2001 and references therein). The present theoretical framework provides a road-map for interpreting the basic elements entering such classical models from the atomic and molecular level, without altering the useful conceptual language developed from those models over more than 50 years of electrophysiology.

Considering only the permeant ions as the most relevant dynamical variables has obvious advantages, but also some pitfalls. Effectively, this is valid only if there are no slow processes associated with the solvent or the protein and that the memory function decays very rapidly compared to all time-scales relevant to ion permeation (see Fig. 3). This is probably a reasonable approximation in the case of $\mathrm{gA}, \mathrm{KcsA}$ and $\mathrm{OmpF}$, as long as gating processes are not considered explicitly. For times long compared to the permeation of a single ion, these channels undergo small and rapid atomic fluctuations in the order of 0.5-1.0 A RMS around well-defined structures and the progression from Eq. (3) to Eq. (8). is valid. If inertial and memory effects become important, it is possible to use Eq. (3) instead of Eq. (8). In more complex cases, it might become necessary to include some protein and solvent degrees of freedom explicitly into the set of relevant variables. For example, the isomerization of acidic side-chains, as well 
as the permeating $\mathrm{Cl}^{-}$ions must be included in the set of relevant variables to describe the concentration-dependent gating properties of the ClC channel (Dutzler et al. 2002). Furthermore, there are some indications that the orientation of the water molecules in the single-file region of gA could be a slow variable which may need to be considered explicitly (Schumaker et al. 2000, 2001; Allen et al. 2003b). The current framework, based on the Mori-Zwanzig projection operator formalism (Zwanzig, 2001), can be extended to incorporate all these other 'relevant' degrees of freedom, if necessary. Alternatively, it is possible to use special techniques to determine multi-atom reaction coordinates for the permeation process (Fischer \& Karplus, 1992; Siva \& Elber, 2003). The genuine dynamical path linking two stable states can be sampled and characterized in its full details using the powerful transition path sampling (TPS) method developed by Chandler (Bolhuis et al. 2000; Bolhuis \& Chandler, 2000). One can anticipate that such considerations will become increasingly important when examining large conformational transitions associated with gating processes. Undoubtedly, one day computers will be sufficiently powerful to allow the direct simulation of non-equilibrium ion fluxes from MD (Crozier et al. 2001a, b; Yang et al. 2003). But even then, the basic elements discussed in sections $2-5$ will continue to provide a fundamental conceptual framework to rigorously 'translate' the properties of the atomic level into the language of simple phenomenological permeation models.

\section{Acknowledgments}

This work was supported by NIH grant no. GM62342 (B.R.) and by the Revson and Keck Foundations (T.W.A.). We are grateful to Olaf S. Andersen for insightful comments and his support of T.W.A. through NIH grant no. GM21342. Comments on the manuscript by Jose Faraldo-Gomez, Vishwanath Jogini, Esin Kutluay, Guillaume Lamoureux, Sergey Noskov and Peter Tieleman are gratefully acknowledged.

\section{References}

Adelman, S. A. (1980). Generalized Langevin equation and many-body problems in chemical dynamics. Advances in Chemical Physics 44, 143-253.

Agmon, N. \& Hopfield, J. J. (1983). Transient kinetics of chemical-reactions with bounded diffusion perpendicular to the reaction coordinate - intramolecular processes with slow conformational-change. Journal of Chemical Physics 78, 6947-6959.

Allen, M. P. \& Tildesley, D. J. (1989). Computer Simulation of Liquids. Oxford: Oxford Science Publications, Clarendon Press.

Allen, T. W., Andersen, O. S. \& Roux, B. (2003a). The structure of gramicidin A in a lipid bilayer environment determined using molecular dynamics simulations and solid-state NMR data. Journal of the American Chemical Society 125, 9868-9877.

Allen, T. W., Andersen, O. S. \& Roux, B. (2003b). Energetics of ion conduction through the gramicidin channel. Proceedings of the National Academy of Sciences USA 101, 117-122.

Allen, T. W., Bastug, T., Kuyucak, S. \& Chung, S. H. (2003c). Gramicidin a channel as a test ground for molecular dynamics force fields. Biophysical Journal 84, 2159-2268.

Allen, T. W., Bliznyuk, A., Rendell, A. P., Kuyucak, S. \& Chung, S. H. (2000). The potassium channel: structure, selectivity and diffusion. Journal of Chemical Physics 112, 8191-8204.

Allen, T. W. \& Chung, S. H. (2001). Brownian dynamics of an open-state KcsA potassium channel. Biophysica et Biochemica Acta 1515, 83-91.

Allen, T. W., Kuyucak, S. \& Chung, S. H. (1999). Molecular dynamics study of the KcsA potassium channel. Biophysical Journal 77, 2502-2516.

Andersen, O.S. (1983). Ion movement through gramicidin A channels. Interfacial polarization effects on single-channel measurements. Biophysical Journal 41, 135-146.

Andersen, O. S. \& Koeppe, R. E. (1992). Molecular determinants of channel function. Physiological Review 72, S89-S158.

Andersen, O. S. \& Procopio, J. (1980). Ion movement through gramicidin A channels. Acta Physiologica (Suppl.) 481, 27-35. 
ÅQvist, J. \& Luzhkov, V. (2000). Ion permeation mechanism of the potassium channel. Nature 404, 881-884.

Arseniev, A. S., Barsukov, I. L., Bystrov, V. F., Lomize, A. L. \& OvchinNikov, Y. A. (1985). ${ }^{1}$ H-NMR study of gramicidin A transmembrane ion channel Head-tohead, right-handed, single-stranded helices. FEBS Letters 186, 168-174.

BALESCU, R. (1975). Equilibrium and Non-equilibrium Statistical Mechanics. John Wiley and Sons, New York.

Banavali, N. K. \& Roux, B. (2002). Atomic radii for continuum electrostatics calculations on nucleic acids. Journal of Physical Chemistry (B) 106, 11026-11035.

Becker, M. D., Koeppe, R. E. \& Andersen, O. S. (1992). Amino acid substitutions and ion channel function. Model-dependent conclusions. (Biophysial Discussions) Biophysical Journal 62, 25-27.

Becker, O. M., MacKerell, A. D., Roux, B. \& Watanabe, M. (eds.) (2001). Computational Biochemistry and Biophysics. New York: Marcel Dekker.

Beglov, D. \& Roux, B. (1995). Numerical solution of the HNC equation for a solute of arbitrary geometry in three dimensions. Journal of Chemical Physics 103, 360-364.

Beglov, D. \& Roux, B. (1996). Solvation of complex molecules in a polar liquid: an integral equation theory. Journal of Chemical Physics 104, 8678-8689.

Beglov, D. \& Roux, B. (1997). An integral equation to describe the solvation of polar molecules in liquid water. Journal of Physical Chemistry (B) 101, 7821-7826.

BeK, S. \& JAKOBsson, E. (1994). Brownian dynamics study of a multiply-occupied cation channel: application to understanding permeation in potassium channels. Biophysical Journal 66, 1028-1038.

Berard, D. R. \& Patey, G. N. (1992). A mean fieldtheory for fluids of multipolar particles in contact with a polarizable wall. Journal of Chemical Physics 97, 4372-7379.

Berne, B. J., Borkovec, M. \& Straub, J. E. (1988). Classical and modern methods in reaction rate theory. Journal of Physical Chemistry 92, 3711-3725.

Berne, B. J., Tuckerman, M. E., Straub, J. E. \& Bug, A. L. R. (1990). Dynamic friction on rigid and flexible bonds. Journal of Chemical Physics 93, 5084-5095.

Bernèche, S. \& Roux, B. (2000). Molecular dynamics of the $\mathrm{KcsA} K(+)$ channel in a bilayer membrane. Biophysical Journal 78, 2900-2917.

Bernèche, S. \& Roux, B. (2001). Energetics of ion conduction through the $\mathrm{K}^{+}$channel. Nature 414, 73-77.

Bernèche, S. \& Roux, B. (2003). A microscopic view of ion conduction through the KcsA $\mathrm{K}^{+}$channel. Proceedings of the National Academy of Sciences USA 100, 8644-8648.

Biggin, P. C., Smith, G. R., Shrivastava, I., Choe, S. \& SAnsom, M. S. (2001). Potassium and sodium ions in a potassium channel studied by molecular dynamics simulations. Biochimica et Biophysica Acta 1510, 1-9.
Bjorksten, J., Soares, C. M., Nilsson, O. \& Tapia, O. (1994). On the stability and plastic properties of the interior L3 loop in R. capsulatus porin. A molecular dynamics study. Protein Engineering 7, 487-493.

Bockris, J. O. M. \& Reddy, A. K. N. (1970). Moderm Electrochemistry. London: McDonald.

Boczko, E. M. \& Brooks III., C. L. (1993). Constanttemperature free energy surfaces for physical and chemical processes. Journal of Physical Chemistry 97, 4509-4513.

Bolmuis, P. G. \& Chandler, D. (2000). Transition path sampling of cavitation between molecular scale solvophobic surfaces. Journal of Chemical Physics 113, 8154 8160 .

Bolhuis, P. G., Dellago, C. \& Chandler, D. (2000). Reaction coordinates of biomolecular isomerization. Proceedings of the National Academy of Sciences USA 97, 5877-5882.

Bond, P. J., Faraldo-Gomez, J. D. \& Sansom, M. S. (2002). OmpA: a pore or not a pore? Simulation and modeling studies. Biophysical Journal 83, 763-775.

Born, M. (1920). Volumen and hydratationswarme der ionen. Zeitschrift für Physik 1, 45-48.

Brewer, M. L., Schmitt, U. W. \& Voth, G. A. (2001). The formation and dynamics of proton wires in channel environments. Biophysical Journal 80, 1691-1702.

Brooks, B. R., Bruccoleri, R. E., Olafson, B. D., States, D. J., Swaminathan, S. \& Karplus, M. (1983). CHARMM: a program for macromolecular energy minimization and dynamics calculations. Journal of Computational Chemistry 4, 187-217.

Burykin, A., Kato, M. \& Warshel, A. (2003). Exploring the origin of the ion selectivity of the KcsA potassium channel. Proteins: Structure, Function, Genetics 52, 412-426.

Burykin, A., Schutz, C. N., Villa, J. \& Warshel, A. (2002). Simulations of ion current in realistic models of ion channels: the KcsA potassium channel. Proteins: Structure, Function, Genetics 47, 265-280.

Calef, D. \& Wolynes, P. G. (1983). SmoluchowskyVlasov theory of charge solvation dynamics. Journal of Chemical Physics 78, 4145-4153.

Capener, C. E., Shrivastava, I. H., Ranatunga, K. M., Forrest, L. R., Smith, G. R. \& SAnsom, M. S. (2000). Homology modeling and molecular dynamics simulation studies of an inward rectifier potassium channel. Biophysical Journal 78, 2929-2942.

Cardenas, A. E., Coalson, R. D. \& Kurnikova, M. G. (2000). Three-dimensional Poisson-Nernst-Planck theory studies: influence of membrane electrostatics on gramicidin A channel conductance. Biophysical Journal 79, 80-93.

Chandler, D. (1977). The dielectric constant and related properties of molecular fluids: interaction cluster theory analysis. Journal of Chemical Physics 67, 1113-1124.

Chandrasekar, S. (1943). Stochastic problem in physics and astronomy. Reviews of Modern Physics 15, 1-89. 
Chang, G., Spencer, R. H., Lee, A. T., Barclay, M. T. \& ReEs, D. C. (1998). Structure of the mscl homolog from mycobacterium tuberculosis: a gated mechanosensitive ion channel. Science 282, 2220-2226.

Chapman, D. L. (1913). A contribution to the theory of electrocapillarity. Philosophical Magazine 25, 475-481.

Chiu, S. W. \& Jakobsson, E. (1989). Stochastic theory of singly occupied ion channels. II. Effects of access resistance and potential gradient extending into the bath. Biophysical Journal 55, 147-157.

Chiu, S. W., Novotny, J. A. \& Jakobsson, E. (1993). The nature of ion and water barrier crossing in a simulated ion channel. Biophysical Journal 64, 98-109.

Chiu, S. W., Subramaniam, S. \& Jakobsson, E. (1999a). Simulation study of a gramicidin/lipid bilayer system in excess water and lipid. I. Structure of the molecular complex. Biophysical Journal 76, 1929-1938.

Chiu, S. W., Subramaniam, S. \& Jakobsson, E. (1999b). Simulation study of a gramicidin/lipid bilayer system in excess water and lipid. II. Rates and mechanisms of water transport. Biophysical Journal 76, 1939-1950.

Chung, S. H., Allen, T.W. \& Kuyucak, S. (2002). Modeling diverse range of potassium channels with Brownian dynamics. Biophysical Journal 83, 263-277.

Chung, S. H., Hoyles, M., Allen, T. W. \& Kuyucak, S. (1998). Study of ionic currents across a model membrane channel using Brownian dynamics. Biophysical Journal 75, 793-809.

Chung, S. H. \& Kuyucak, S. (2002). Recent advances in ion channel research. Biocbimica et Biophysica Acta 1565, 267-286.

Colombo, G., Marrink, S. J. \& Mark, A. E. (2003). Simulation of MscL gating in a bilayer under stress. Biophysical Journal 84, 2331-2337.

Cooper, K. E., Jakobsson, E. \& Wolynes, P. G. (1985). The theory of ion transport through membrane channels. Progress in Biophysics and Molecular Biology 46, 51-96. Cornell, W. D., Cieplak, P., Bayly, C. I., Gould, I. R., Merz Jr., K. M., Ferguson, D. M., Spellmeyer, D. C., Fox, T., Caldwell, J. W. \& Kollman, P. A. (1995). A second generation force field for the simulation of proteins and nucleic acids. Journal of the American Chemical Society 117, 5179-5197.

Corry, B., Kuyucak, S. \& Chung, S. H. (2000). Tests of continuum theories as models of ion channels. II. Poisson-Nernst-Planck theory versus Brownian dynamics. Biophysical Journal 78, 2364-2381.

Cortes, D. M., Cuello, L. G. \& Perozo, E. (2001). Molecular architecture of full-length KcsA: role of cytoplasmic domains in ion permeation and activation gating. Journal of General Physiology 117, 165-180.

Cortis, C. M., Rossky, P. J. \& Friesner, R. A. (1997). A three-dimensional reduction for the Orstein-Zernicke equation for molecular liquids. Journal of Chemical Physics 107, 6400-6414.
Cowan, S. W., Schirmer, T., Rummel, G., Steiert, M., Gosh, R., Pauptit, R. A., Jansonius, J. N. \& Rosenbusch, J. P. (1992). Crystal structures explain functional properties of two E. coli porins. Nature 358, $727-733$.

Cox, B. G., Hedwig, G. R., Parker, A. J. \& Watts, D. W. (1974). Solvation of ions XIX. Thermodynamic properties for transfer of single ions between protic and dipolar aprotic solvents. Australian Journal of Chemistry 27, 477-501.

Crouzy, S., Bernèche, S. \& Roux, R. (2001). Extracellular blockade of $\mathrm{K}^{+}$channels by TEA: results from molecular dynamics simulations of the KcsA channel. Journal of General Physiology 118, 207-217.

Crouzy, S., Woolf, T. B. \& Roux, B. (1994). A molecular dynamics study of gating in dioxolane-linked gramicidin A channels. Biophysical Journal 67, 1370-1386.

Crozier, P. S., Henderson, D., Rowley, R. L. \& Busath, D. D. (2001a). Model channel ion currents in $\mathrm{NaCl}$ extended simple point charge water solution with applied-field molecular dynamics. Biophysical Journal 81, 3077-3389.

Crozier, P. S., Rowley, R. L., Holladay, N. B., Henderson, D. \& Busath, D. D. (2001b). Molecular dynamics simulation of continuous current flow through a model biological membrane channel. Physical Review Letters 86, 2467-2470.

Danelon, C., Suenaga, A., Winterhalter, M. \& Yamato, I. (2003). Molecular origin of the cation selectivity in OmpF porin: single channel conductances vs. free energy calculation. Biophysical Chemistry 104, 591-603.

Davis, M. E., Madura, J. D., Luty, B. \& McCammon, J. A. (1991). Electrostatic and diffusion of molecules in solution: simultations with the University of Houston Brownian Dynamics program. Comparative Physics Communications 62, 187-197.

Debye, P. \& HüCKel, E. (1923). Zur Theorie der Elektrolyte. II. Das Grenzgesetz für die elktrische Leitfähigkeit. Physikalische Zeitschrift 24, 305-325.

deGroot, B. L., Tieleman, D. P., Pohl, P. \& Grubmuller, H. (2002). Water permeation through gramicidin A: desformylation and the double helix: a molecular dynamics study. Biophysical Journal 82, 2934-2942.

Deleeuw, S. W., Perram, J. W. \& Smith, E. R. (1980). Simulation of electrostatic systems in periodic boundary-conditions. 1. Lattice sums and dielectric-constants. Proceedings of the Royal Society of London (A) 373, 27-56.

Dorman, V., Partenskit, M. B. \& Jordan, P. C. (1996). A semi-microscopic Monte Carlo study of permeation energetics in a gramicidin-like channel: the origin of cation selectivity. Biophysical Journal 70, 121-134.

Doyle, D. A., Cabral, J. M., Pfuetzner, R. A., Kuo, A., Gulbis, J. M., Cohen, S. L., Chait, B. T. \& MacKINNON, R. (1998). The structure of the potassium channel: molecular basis of $\mathrm{K}^{+}$conduction and selectivity. Science 280, 69-77. 
Du, Q., Beglov, D. \& Roux, B. (1999). Hydration free energy of polar and non-polar molecules: an extended site-reduced integral equation theory. Journal of Physical Chemistry (B) 104, 796-805.

Dutzler, R., Campbell, E. B., Cadene, M., Chait, B. T. \& MacKinnon, R. (2002). X-ray structure of a CIC chloride channel at 3.0 A reveals the molecular basis of anion selectivity. Nature 415, 287-294.

Dutzler, R., Rummel, G., Alberti, S., Hernandez-Alles, S., Phale, P., Rosenbusch, J., Benedi, V. \& Schirmer, T. (1999). Crystal structure and functional characterization of OmpK36, the osmoporin of Klebsiella pneumoniae. Structure 7, 425-434.

Edwards, S., Corry, B., Kuyucak, S. \& Chung, S. H. (2002). Continuum electrostatics fails to describe ion permeation in the gramicidin channel. Biophysical Journal 83, 1348-1360.

Einstein, A. (1926). Investigation on the Theory of Brownian Movement. Translation by A. D. Cowper of papers published 1905-1908. New York: Dover Publications.

Eisenman, G. (1962). Cation selective electrodes and their mode of operation. Biophysical Journal 2 (Suppl. 2), 259-323.

Elber, R., Chen, D. P., Rojewska, D. \& Eisenberg, R. (1995). Sodium in gramicidin: an example of a permion. Biophysical Journal 68, 906-924.

Elmore, D. E. \& Dougherty, D. A. (2001). Molecular dynamics simulations of wild-type and mutant forms of the Mycobacterium tuberculosis MscL channel. Biophysical Journal 81, 1345-1359.

ERMAK, D. L. (1975). A computer simulation of charged particles in solution. I. Technique and equilibrium properties. Journal of Chemical Physics 62, 4162-4196.

Everitt, C. T. \& Haydon, D. A. (1968). Electrical capacitance of a lipid membrane separating two aqueous phases. Journal of Theoretical Biology 18, 371-379.

Faraldo-Gomez, J. D., Smith, G. R. \& Sansom, M. S. (2002). Setting up and optimization of membrane protein simulations. European Biophysical Journal 31, 217-227.

Faraldo-Gomez, J. D., Smith, G. R. \& Sansom, M. S. (2003). Molecular dynamics simulations of the bacterial outer membrane protein FhuA: a comparative study of the ferrichrome-free and bound states. Biophysical Journal 85, 1406-1420.

Feller, S. E., Pastor, R. W., Rojnuckarin, A., Bogusz, S. \& Brooks, B. R. (1996). Effect of electrostatic force truncation on interfacial and transport properties of water. Journal of Physical Chemistry 100, 17011-17020.

Finkelstein, A. \& Andersen, O.S. (1981). The Gramicidin A Channel: a review of its permeability characteristics with special reference to the singlefile aspect of transport. Journal of Membrane Biology 59, 155-171.

Fischer, S. \& Karplus, M. (1992). Conjugate peak refinement - an algorithm for finding reaction paths and accurate transition-states in systems with many degrees of freedom. Chemical Physics Letters 194, 252-261.

Fischer, W., Brickmann, J. \& Läuger, P. (1981). Molecular dynamics study of ion transport in transmembrane protein channels. Biophysical Chemistry 13, 105-116.

Forrest, L. R., Kukol, A., Arkin, I. T., Tieleman, D. P. \& SANSOM, M. S. (2000). Exploring models of the influenza A M2 channel: MD simulations in a phospholipid bilayer. Biophysical Journal 78, 55-69.

Fowler, R. H. \& Guggenheim, E. A. (1939). Statistical Thermodynamics. Cambridge University Press.

Fries, P. H. \& Patey, G. N. (1985). The solution of the hypernetted-chain approximation for fluids of nonspherical particles - a general-method with application to dipolar hard-spheres. Journal of Chemical Physics 82, 429-440.

Garofoli, S. \& Jordan, P. C. (2003). Modeling permeation energetics in the KcsA potassium channel. Biophysical Journal 84, 2814-2830.

Gennis, R. B. (1989). Biomembranes: Molecular Structure and Functions. New York: Springer-Verlag.

Gillespie, D., Nonner, W. \& Eisenberg, R. S. (2002). Coupling Poisson-Nernst-Planck and density functional theory to calculate ion flux. Journal of Physics: Condensed Matter 14, 12129-12145.

Gilson, M. K. \& Honig, B. H. (1986). The dielectric constant of a folded protein. Biopolymers 25, 2097-2119.

Gilson, M. K., Sharp, K. A. \& Honig, B. H. (1987). Calculating the electrostatic potential of molecules in solution-method and error assessment. Journal of Comparative Chemistry 9, 327-335.

Goldman, D. E. (1943). Potential, impedence and rectification in membranes. Journal of General Physiology 27, $37-60$.

Gouy, G. (1910). Sur la constitution de la charge electrique a la surface d'un electrolyte. Journal of Physiolog (Paris), 9, 457-468.

Graf, P., Nitzan, A., Kurnikova, M. G. \& Coalson, R. D. (2000). A dynamic lattice Monte Carlo model of ion transport in inhomogeneous dielectric environments: method and implementation. Journal of Physical Chemistry (B) 104, 12324-12338.

Gross, A., Columbus, L., Hideg, K., Altenbach, C. \& Hubbell, W. L. (1999). Structure of the KcsA potassium channel from Streptomyces lividans: a site-directed spin labeling study of the second transmembrane segment. Biochemistry 38, 10324-11035.

Gross, A. \& Hubbell, W. L. (2002). Identification of protein side chains near the membrane-aqueous interface: a site-directed spin labeling study of KcsA. Biochemistry 41, 1123-1128.

GuÀrdia, E. \& PARdRó, J. (1991). Potential of mean force by constrained molecular dynamics: a sodium chloride ion-pair in water. Chemical Physics 155, 187-195. 
Guidoni, L. \& Carloni, P. (2002). Potassium permeation through the KcsA channel: a density functional study. Biochimica et Biophysica Acta 1563, 1-6.

Guidoni, L., Torre, V. \& Carloni, P. (1999). Potassium and sodium binding to the outer mouth of the $\mathrm{K}^{+}$ channel. Biochemistry 38, 8599-8604.

Guidoni, L., Torre, V. \& Carloni, P. (2000). Water and potassium dynamics inside the KcsA $\mathrm{K}(+)$ channel. FEBS Letters 477, 37-42.

Gullingsrud, J., Kosztin, D. \& Schulten, K. (2001). Structural determinants of MscL gating studied by molecular dynamics simulations. Biophysical Journal 80, 2074-2081.

Gullingsrud, J. \& Schulten, K. (2003). Gating of MscL studied by steered molecular dynamics. Biophysical Journal 85, 2087-2299.

Halgren, T. A. \& Damm, W. (2001). Polarizable force fields. Current Opinion in Structural Biology 11, 236-242.

Hansen, J. P. \& McDonald, I. R. (1986). Theory of Simple Liquids, 2nd edn. London: Academic Press.

Heffelfinger, G. S. \& Ford, D. M. (1998). Massively parallel dual control volume grand canonical molecular dynamics with ladera $\mathrm{i}$-gradient driven diffusion in Lennard-Jones fluids. Molecular Physics 94, 659-671.

Heginbotham, L., LeMasurier, M., KolmakovaPartensky, L. \& Miller, C. (1999). Single Streptomyces lividans $\mathrm{K}(+)$ channels. Functional asymmetries and sidedness of proton activation. Journal of General Physiology 114, 551-560.

Helfand, E. (1960). Transport coefficient from dissipation in a canonical ensemble. Physics Review 119, 1-9.

Hille, B. (2001). Ionic Channels of Excitable Membranes, 3rd edn. Sunderland, MA: Sinauer.

Hille, B. \& Schwarz, W. (1978). Potassium channels as multi-ion single-file pores. Journal of General Physiology 72, 409-442.

Hinton, J. F., Fernandez, J. Q., Shungu, D. C., Whaley, W. L., Koeppe II., R. E. \& Millett, F. S. (1988). TI-205 nuclear magnetic resonance determination of the thermodynamic parameters for the binding of monovalent cations to Gramicidin A and C. Biophysical Journal 54, $527-533$.

Hinton, J. F., Whaley, W. L., Shungu, D. C., Koeppe II., R. E. \& Millett, F. S. (1986). Equilibrium binding constant for the group I metal cations with gramicidinA determined by competition studies and $\mathrm{T}^{+}{ }^{+}-205$ nuclear magnetic resonance spectroscopy. Biophysical Journal 50, 539-544.

Hirata, F., Redfern, P. \& Levy, R. M. (1988). Viewing the born model for ion hydration through a microscope. International Journal of Quantum Chemistry: Quantum Biology Symposium 15, 179.

Hodgkin, A. L. \& Katz, B. (1949). The effect of sodium ions on the electrical activity of the giant axon of the squid. Journal of Physiology (London) 108, 37-77.
Hodgkin, A. L. \& Keynes, R. D. (1955). The potassium permeability of a giant nerve fibre. Journal of Physiology (London) 128, 61-88.

Hollerbach, U., Chen, D. P., Busath, D. D. \& Eisenberg, B. (2000). Predicting function from structure using the Poisson-Nernst-Planck equations: sodium current in the gramicidin A channel. Langmuir 16, 5509-5514.

Hollerbach, U., Chen, D. P., Busath, D. D. \& Eisenberg, B. (2002). Concentration-dependent shielding of electrostatic potentials inside the gramicidin A channels. Langmuir 18, 3626-3631.

Honig, B. \& Nicholls, A. (1995). Classical electrostatics in biology and chemistry. Science 268, 1144.

Hunenberger, P. H. \& McCammon, J. A. (1999). Effect of artificial periodicity in simulations of biomolecules under Ewald boundary conditions: a continuum electrostatics study. Biophysical Chemistry 78, 69-88.

Im, W., Beglov, D. \& Roux, B. (1998). Continuum solvation model: electrostatic forces from numerical solutions to the Poisson-Bolztmann equation. Comparative Physics Communications 111, 59-75.

Im, W. \& Roux, B. (2001). Brownian dynamics solutions of ions channels: a general treatment of electrostatic reaction fields for molecular pores arbitrary geometry. Journal of Chemical Physics 115, 4850-4861.

Im, W. \& Roux, B. (2002a). Ion permeation and selectivity of OmpF porin: a theoretical study based on molecular dynamics, Brownian dynamics, and continuum electrodiffusion theory. Journal of Molecular Biology 322, 851-869.

Im, W. \& Roux, B. (2002b). Ions and counterions in a biological channel: a molecular dynamics simulation of OmpF porin from Escherichia coli in an Explicit membrane with $1 \mathrm{M} \mathrm{KCl}$ aqueous salt solution. Journal of Molecular Biology 319, 1177-1197.

Im, W., Seefeld, S. \& Roux, B. (2000). A Grand Canonical Monte Carlo - Brownian Dynamics algorithm for simulating ion channels. Biophysical Journal 79, 788-801.

JACKson, J. D. (1962). Classical Electrodynamics. New York: John Wiley \& Sons.

Jakobsson, E. \& Chiu, S. W. (1987). Stochastic theory of ion movement in channels with single-ion occupancy. Application to sodium permeation of gramicidin channels. Biophysical Journal 52, 33-45.

Jardat, M., Bernard, O., Turq, P. \& Kneller, G. R. (1999). Transport coefficients of electrolyte solutions from Smart Brownian dynamics simulations. Journal of Chemical Physics 110, 7993-7999.

Jayaram, B., Fine, R., Sharp, K. \& Honig, B. (1989). Free energy calculations of ion hydration: an analysis of the born model in terms of microscopic simulations. Journal of Physical Chemistry 93, 4320.

Jean-Charles, A., Nicholls, A., Sharp, K., Honig, B., Tempczyk, A., Hendrickson, T. \& Still, W. C. (1991). 
Electrostatic contributions to solvation energies comparison of free-energy perturbation and continuum calculations. Journal of the American Chemical Society 113, 1454-1455.

Jiang, Y., Lee, A., Chen, J., Cadene, M., Chait, B. T. \& MacKinnon, R. (2002a). Crystal structure and mechanism of a calcium-gated potassium channel. Nature $417,515-522$

Jiang, Y., Lee, A., Chen, J., Cadene, M., Chait, B. T. \& MacKinnon, R. (2002b). The open pore conformation of potassium channels. Nature 417, 523-526.

Jiang, Y., Lee, A., Chen, J., Ruta, V., Cadene, M., Chait, B. T. \& MAcKinnon, R. (2003). X-ray structure of a voltage-dependent $\mathrm{K}^{+}$channel. Nature 423, 33-41.

Jiang, Y. \& MacKinnon, R. (2000). The barium site in a potassium channel by X-ray crystallography. Journal of General Physiology 115, 269-272.

Jing, N., Prasad, K. U. \& Urry, D. W. (1995). The determination of binding constants of micellar-packaged gramicidin A by $13 \mathrm{C}$-and 23Na-NMR. Biocbimica et Biophysica Acta 1238, 1-11.

JoRDAN, P. C. (1981). Energy barriers for passage of ions through channels. Exact solution of two electrostatic problems. Biophysical Chemistry 13, 203-212.

Jordan, P. C. (1984). The total electrostatic potential in a gramicidin channel. Journal of Membrane Biology 78, 91-102.

JoRDAN, P. C. (1987). Microscopic approach to ion transport through transmembrane channels. The model system gramicidin. Journal of Physical Chemistry 91, 6582-6591.

Jordan, P. C., Bacquet, R. J., McCammon, J. A. \& Tran, P. (1989). How electrolyte shielding influences the electrical potential in transmembrane ion channels. Biophysical Journal 55, 1041-1052.

Jordan, P. C. \& VAYL, S. (1985). How shortening a channel may lower its conductance. The case of des$\mathrm{Val}^{7}-d \mathrm{Val}^{8}$-gramicidin A. Biochimica et Biophysica Acta 818, 416-420.

Karshikoff, A., Spassov, A., Cowan, S. W., Ladenstein, R. \& Schirmer, T. (1994). Electrostatic properties of two porin channels from E. coli. Journal of Molecular Biology 240, 372-384.

Kelly, B. L. \& Gross, A. (2003). Potassium channel gating observed with site-directed mass tagging. Nature Structural Biology 10, 280-284.

Ketchem, R. R., Hu, W. \& Cross, T. A. (1993). Highresolution conformation of gramicidin A in lipid bilayer by solid-state NMR. Science 261, 1457-1460.

Ketchem, R. R., Roux, B. \& Cross, T. A. (1997). High resolution refinement of a solid-state NMR-derived structure of gramicidin A in a lipid bilayer environment. Structure 5, 11655-11669.

Kim, S., Quine, J. R. \& Cross, T. A. (2001). Complete cross-validation and R-factor calculation of a solid-state NMR derived structure. Journal of the American Chemical Society 123, 7292-7298.
King, G., Lee, F. S. \& Warshel, A. (1991). Microscopic simulation of macroscopic dielectric constants of solvated proteins. Journal of Chemical Physics 95, 4365-4377.

KIRKWOOD, J. G. (1934). Theory of solution of molecules containing widely separated charges with application to zwiterions. Journal of Chemical Physics 2, 351.

Kirkwood, J. G. (1935). Statistical mechanics of fluid mixtures. Journal of Chemical Physics 3, 300-313.

Klapper, I., Hagstrom, R., Fine, R., Sharp, K. \& Honig, B. (1986). Focusing of electric fields in the active site of $\mathrm{Cu}-\mathrm{Zn}$ superoxide dismutase: effects of ionic strength and amino-acid modification. Proteins 1, 47.

Kollman, P. A. (1993). Free energy calculations: applications to chemical and biochemical phenomena. Chemical Review 93, 2395-2417.

Koumanov, A., Zachariae, U., Engelhardt, H. \& Karshikoff, A. (2003). Improved 3D continuum calculations of ion flux through membrane channels. European Biophysics Journal 32, 689-702.

Kovalenko, A. \& Hirata, F. (1998). Three-dimensional density profiles of water in contact with a solute of arbitrary shape-a RISM approach. Chemical Physics Letters 290, 237-244.

Kubo, R. (1966). The fluctuation-dissipation theorem. Reviews in Modern Physics 29, 255-284.

Kumar, S., Bouzida, D., Swendsen, R. H., Kollman, P. A. \& Rosenberg, J. M. (1992). The Weighted Histogram Analysis Method for free-energy calculations on biomolecules. I. The method. Journal of Comparative Chemistry 13, 1011-1021.

Kuo, A., Gulbis, J. M., Antcliff, J. F., Rahman, T., Lowe, E. D., Zimmer, J., Cuthbertson, J., Ashcroft, F. M., Ezaki, T. \& Doyle, D. A. (2003). Crystal structure of the potassium channel KirBac1.1 in the closed state. Science 300, 1922-1926.

Kurnikova, M. G., Coalson, R. D., Graf, P. \& Nitzan, A. (1999). A lattice relaxation algorithm for threedimensional Poisson-Nernst-Planck theory with application to ion transport through the gramicidin A channel. Biophysical Journal 76, 642-656.

Kusalik, P. G. \& Patey, G. N. (1988). On the molecular theory of aqueous-electrolyte solutions. 1. The solution of the RHNC approximation for models at finite concentration. Journal of Chemical Physics 88, 7715-7738.

Kutluay, E., Roux, B. \& Heginbotham, L. (In Press). Intracellular blockade of KcsA by tea. Journal of General Physiology.

Kuyucak, S., Andersen, O. S. \& Chung, S. H. (2001). Models of permeation in ion channels. Reports on Progress in Physics 64, 1427-1472.

Lamoureux, G., MacKerell, A. D. \& Roux, B. (2003). A simple water model with Drude-oscillator polarizability. Journal of Chemical Physics 119, 5185-5197.

LäUger, P. (1973). Ion transport through pores: a rate theory analysis. Biochimica et Biophysica Acta 311, 423-441. 
Läuger, P., Lesslauer, W., Marti, E. \& Richter, J. (1967). Electrical properties of bimolecular phospholipid membranes. Biochimica et Biophysica Acta 135, 20-32.

Lebowitz, J. L. \& Percus, J. K. (1966). Mean spherical model of lattice gases with extended hard cores and continuum fluids. Physics Review 144, 251.

LeMasurier, M., Heginbotham, L. \& Miller, C. (2001). KcsA: it's a potassium channel. Journal of General Physiology 118, 303-314.

LevitT, D. G. (1978). Electrostatic calculations for an ion channel. I. Energy and potential profiles and interactions between ions. Biophysical Journal 22, 209-219.

LevitT, D. G. (1986). Interpretation of biological ion channel flux data-Reaction-rate theory versus continuum theory. Annual Review of Biophysics and Biophysical Chemistry 15, 29-57.

LevitT, D. G. (1999). Modeling of ion channels. Jorunal of General Physiology 113, 789-794.

Liu, Y. S., Sompornpisut, P. \& Perozo, E. (2001). Structure of the KcsA channel intracellular gate in the open state. Nature Structure Biology 8, 883-887.

Llano-Restrepo, M. \& Chapman, W. (1994). Monte carlo simulation of the structural properties of concentrated aqueous alkali halide solutions at $25^{\circ}$ using a simple civilized model. Journal of Chemical Physics 100, 83218339.

Luzhkov, V. B. \& ÅQvist, J. (2000). A computational study of ion binding and protonation states in the KcsA potassium channel. Biochimica et Biophysica Acta 1481, 360-370.

Luzhkov, V. B. \& Åqvist, J. (2001a). K(+)/NA(+) selectivity of the KcsA potassium channel from microscopic free energy perturbation calculations. Biochimica et Biophysica Acta 1548, 194-202.

Luzhkov, V. B. \& ÅQvist, J. (2001b). Mechanisms of tetraethylammonium ion block in the KcsA potassium channel. FEBS Letters 495, 191-196.

Lyubartsev, A. P. \& Laaksonen, A. (1996). Concentration effects in aqueous nacl solutions: a molecular dynamics simulation. Journal of Chemical Physics 100, 16410-16418.

Mackay, D. H., Berens, P. H. \& Wilson, K. R. (1984). Structure and dynamics of ion transport through Gramicidin A. Biophysical Journal 46, 229-248.

Mackay, D. H. \& Wilson, K. R. (1986). Possible allosteric significance of water structures in proteins. Journal of Biomolecular and Structural Dynamics 4, 491-500.

MacKerell Jr., A. D., Bashford, D., Bellot, M., Dunbrack, R. L., Evanseck, J. D., Field, M. J., Fischer, S., Gao, J., Guo, H., Joseph-McCarthy, D., Ha, S., Kuchnir, L., Kuczera, K., Lau, F. T. K., Mattos, C., Michnick, S., Ngo, T., Nguyen, D. T., Prodhom, B., Reiher III., W. E., Roux, B., Schlenkrich, M., Smith, J., Stote, R., Straub, J., Watanabe, M., Wiorkiewicz-Kuczera, J. \& Karplus, M. (1998). Allatom empirical potential for molecular modeling and dynamics studies of proteins. Journal of Physical Chemistry (B) 102, 3586-3616.

Mamonov, A. B., Coalson, R. D., Nitzan, A. \& Kurnikova, M. G. (2003). The role of the dielectric barrier in narrow biological channels: a novel composite approach to modeling single-channel currents. Biophysical Journal 84, 3646-3661.

Mashl, R. J., Tang, Y., Schnitzer, J. \& Jakobsson, E. (2001). Hierarchical approach to predicting permeation in ion channels. Biophysical Journal 81, 2473-2483.

Mazur, P. \& Oppenheim, I. (1970). Molecular theory of Brownian motion. Physica 50, 241-258.

Mazur, P. \& Oppenheim, I. (1982). On the exact and phenomenological Langevin equation for a harmonic oscillator in a fluid. Physica 115A, 1-20.

McCammon, J. A., Gelin, B. R. \& Karplus, M. (1977). Dynamics of folded proteins. Nature 267, 585-590.

McCammon, J. A. \& Straatsma, T. P. (1992). Alchemical free energy simulation. Annual Review of Physical Chemistry 43, 407.

MCClesky, E. (1999). Calcium channel permeation: a field in flux. Journal of General Physiology 113, 765-772.

McGill, P. \& Schumaker, M. F. (1996). Boundary conditions for single-ion diffusion. Biophysical Journal 71, 1723-1742.

McQuarrie, D. A. (1976). Statistical Mechanics. New York: Harper and Row.

MiLLeR, C. (1999). Ion hopping defended. Journal of General Physiology 113, 783-787.

Miller, C. (2001). See potassium run. Nature 414, $23-24$.

Milis, R. \& Lobo, V. (1989). Self-Diffusion in Electrolyte Solutions. Amsterdam: Elsevier.

Misra, V. K., Нecht, J. L., Yang, A. S. \& Honig, B. (1998). Electrostatic contributions to the binding free energy of the lambd aci repressor to DNA. Biophysical Journal 75, 2262-2273.

Misra, V. K. \& Honig, B. (1996). The electrostatic contribution to the $\mathrm{B}$ to $\mathrm{Z}$ transition of DNA. Biochemistry 35, 1115-1124.

Morais-Cabral, J. H., Zhou, Y. \& MacKinnon, R. (2001). Energetic optimization of ion conduction rate by the $\mathrm{K}^{+}$selectivity filter. Nature $414,37-42$.

Mori, H. (1965). Transport, collective motion, and Brownian motion. Progress in Theoretical Physics 33, 423-455.

Moy, G., Corry, B., Kuyucak, S. \& Chung, S. H. (2000). Tests of continuum theories as models of ion channels. I. Poisson-Boltzmann theory versus Brownian dynamics. Biophysical Journal 78, 2349-2363.

Nagle, J. F. \& Morowitz, H. J. (1978). Molecular mechanisms for proton transport in membranes. Proceedings of the National Academy of Science USA 75, 298-302.

Nernst, W. (1889). Die elektromotorische Wirksamkeit der Ionen. Zeitschrift für physikalische Chemie 4, 129-181. 
Nernst, W. (1890). Ueber die Erregung von Elektricitat und Warme in Elektrolyten. Annalen der Physik und Chemie (Neue Folge) 39, 161-186.

Neyton, J. \& Miller, C. (1988a). Discrete $\mathrm{Ba}^{2+}$ block as a probe of ion occupancy and pore structure in the high-conductance $\mathrm{Ca}^{2+}$-activated $\mathrm{K}^{+}$channel. Journal of General Physiology 92, 569-586.

Neyton, J. \& Miller, C. (1988b). Potassium blocks barium permeation through a calcium-activated potassium channel. Journal of General Physiology 92, 549-567.

Nienhuis, G. \& Deutch, J. M. (1971). Structure of dielectric fluids. 1. 2-particle distribution function of polar fluids. Journal of Chemical Physics 55, 4213.

Nienhuis, G. \& Deutch, J. M. (1972). Structure of dielectric fluids. 3. Interaction between impurities and dielectric saturation in polar fluids. Journal of Chemical Physics 56, 1819-1834.

Nimigean, C. M. \& Miller, C. (2002). Na(+) block and permeation in a $\mathrm{K}(+)$ channel of known structure. Journal of General Physiology 120, 323-335.

Nina, M., Beglov, D. \& Roux, B. (1997). Atomic radii for continuum electrostatics calculations based on molecular dynamics free energy simulations. Journal of Physical Chemistry (B) 101, 5239-5248.

Nonner, W., Catacuzzeno, L. \& Eisenberg, B. (2000). Binding and selectivity in L-type calcium channels: a mean spherical approximation. Biophysical Journal 79, 1976-1992.

Nonner, W., Chen, D. \& Eisenberg, B. (1999). Progress and prospects in permeation. Journal of General Physiology 113, 773-782.

Noyes, R. M. (1962). Thermodynamics of ion hydration as measure of effective dielectric properties of water. Journal of the American Chemical Society 84, 513-522.

Nyquist, H. (1928). Physics Review 32, 110.

Olah, G. A., Huang, H. W., Liu, W. \& Wu, Y. (1991). The thallium ion distribution in the Gramicidin channel by X-ray diffraction. Journal of Molecular Biology 218, 847.

Onsager, L. (1926). Zur theorie der elektrolyte (1). Physikalische Zeitschrift 27, 388-392.

Onsager, L. (1927). Zur theorie der elekrolyte (2). Physikalische Zeitschrift 28, 277-298.

OnSAGER, L. (1936). Electric moment of molecules in liquids. Journal of the American Chemical Society 58, 1468-1493.

Parsegian, A. (1969). Energy of an ion crossing a low dielectric membrane: solution to four relevant electrostatic problems. Nature 221, 844-846.

Partenskit, M. B. \& Jordan, P. C. (1992a). Nonlinear dielectric behavior of water in transmembrane ion channels: ion energy barriers and the channel dielectric constant. Journal of Physical Chemistry 96, 3906-3910.

Partenskit, M. B. \& Jordan, P. C. (1992b). Theoretical perspectives on ion-channel electrostatics: continuum and microscopic approaches. Quarterly Reviews of Biophysics 25, 477-510.
PAuling, L. (1960). Nature of the Chemical Bond and Structure of Molecules and Crystals, 3rd edn. Ithaca, New York: Cornell University Press.

Percus, J. K. \& Yevick, G. J. (1958). Analysis of classical statistical mechanics by means of collective coordinates. Physics Review 110, 1-13.

Petтiтt, B. M. \& Rossky, P. J. (1986). Alkali halides in water: ion-solvent and ion-ion potential of mean force at infinite dilution. Journal of Chemical Physics 84, 5836-5844.

Phale, P. S., Philippsen, A., Widmer, C., Phale, V. P., Rosenbusch, J. P. \& Schirmer, T. (2001). Role of charged residues at the ompf porin channel constriction probed by mutagenesis and simulation. Biochemistry $\mathbf{4 0}$ 6319-6325.

Pohl, P. I. \& Heffelfinger, G. S. (1999). Massively parallel molecular dynamics simulation of gas permeation across porous silica membranes. Journal of Membrane Science 155, 1-7.

Pomes, R. \& Roux, B. (1996). Structure and dynamics of a proton wire: a theoretical study of $\mathrm{H}^{+}$translocation along the single-file water chain in the gramicidin A channel. Biophysical Journal 71, 19-39.

Pomes, R. \& Roux, B. (1998). Free energy profiles for $\mathrm{H}^{+}$ conduction along hydrogen-bonded chains of water molecules. Biophysical Journal 75, 33-40.

Purcell, E. M. (1977). Life at low Reynolds number. American Journal of Physics 45, 3-11.

Ramanathan, P. S. \& Friedman, H. L. (1971). Study of a refined model for aqueous 1-1 electrolytes. Journal of Chemical Physics 54, 1086-1099.

Ranatunga, K. M., Shrivastava, I. H., Smith, G. R. \& SAnsom, M. S. (2001). Side-chain ionization states in a potassium channel. Biophysical Journal 80, 1210-1219.

Rashin, A. A. \& Honig, B. (1985). Reevaluation of the Born model of ion hydration. Journal of Physical Chemistry 89, 5588 .

Rick, S. W., Stuart, S. J. \& Berne, B. J. (1994). Dynamical fluctuating charge force field: application to liquid water. Journal of Chemical Physics 101, 6141-6156.

Roux, B. (1993). Nonadditivity in cation-peptide interactions: a molecular dynamics and $\mathrm{Ab}$ initio study of $\mathrm{Na}^{+}$ in the gramicidin channel. Chemical Physics Letters 212, 231-240

Roux, B. (1995). The calculation of the potential of mean force using computer simulations. Computer Physics Communications 91, 275-282.

Roux, B. (1996). Valence selectivity of the gramicidin channel: a molecular dynamics free energy perturbation study. Biophysical Journal 71, 3177-3185.

Roux, B. (1997). The influence of the membrane potential on the free energy of an intrinsic protein. Biophysical Journal 73, 2980-2989.

Roux, B. (1999a). Statistical mechanical equilibrium theory of selective ion channels. Biophysical Journal 77, 139-153. 
Roux, B. (1999b). Theories of ion permeation: a chaser. Journal of General Physiology 114, 605-608.

Roux, B. (2002a). Computational studies of the gramicidin channel. Accounts of Chemical Research 35, 366-375.

Roux, B. (2002b). Theoretical and computational models of ion channels. Current Opinion in Structural Biology 12, 182-189.

Roux, B. \& Bernèche, S. (2002). On the potential functions used in molecular dynamics simulations of ion channels. Biophysical Journal 82, 1681-1684.

Roux, B., Bernèche, S. \& Im, W. (2000). Ion channels, permeation and electrostatics: insight into the function of KcsA. Biochemistry 39, 13295-13306.

Roux, B. \& Karplus, M. (1991a). Ion trnsport in a gramicidin-like channel: dynamics and mobility. Journal of Physical Chemistry 95, 4856-4868.

Roux, B. \& Karplus, M. (1991b). Ion transport in a gramicidin-like channel: structure and thermodynamics. Biophysical Journal 59, 961-981.

Roux, B. \& Karplus, M. (1993). Ion transport in the gramicidin channel: free energy of the solvated righthanded dimer in a model membrane. Journal of American Chemical Society 115, 3250-3262.

Roux, B. \& Karplus, M. (1994). Molecular dynamics simulations of the gramicidin channel. Annual Review of Biomolecular and Structural Dynamics 23, 731-761.

Roux, B. \& Karplus, M. (1995). Potential energy function for cations-peptides interactions: an ab initio study. Journal of Comparative Chemistry 16, 690-704.

Roux, B. \& MacKinnon, R. (1999). The cavity and pore helices in the KcsA $\mathrm{K}+$ channel: electrostatic stabilization of monovalent cations. Science 285, 100-102.

Roux, B., Pron'hom, B. \& Karplus, M. (1995). Ion transport in the gramicidin channel: molecular dynamics study of single and double occupancy. Biophysical Journal 68, 876-892.

Roux, B. \& Simonson, T. (1999). Implicit solvent models. Biophysical Chemistry 78, 1-20.

Roux, B., Yu, H. A. \& Karplus, M. (1990). Molecular basis for the Born model of ion solvation. Journal of Physical Chemistry 94, 4683-4688.

Sagnella, D. E., Laasonen, K. \& Klein, M. L. (1996). $\mathrm{Ab}$ initio molecular dynamics study of proton transfer in a polyglycine analog of the ion channel gramicidin A. Biophysical Journal 71, 1172-1178.

Sagnella, D. E. \& Voth, G. A. (1996). Structure and dynamics of hydronium in the ion channel gramicidin A. Biophysical Journal 70, 2043-2051.

Saint, N., Lou, K. L., Widmer, C., Luckey, M., Schirmer, T. \& Rosenbusch, J. P. (1996). Structural and functional characterization of $\mathrm{OmpF}$ porin mutants selected for larger pore size. II. Functional characterization. Journal of Biological Chemistry 271, 20676-20680.

Schirmer, T. (1998). General and specific porins from bacterial outer membranes. Journal of Structural Biology 121, 101-109.
Schirmer, T. \& Phale, P. (1999). Brownian dynamics simulation of ion flow through porin channels. Journal of Molecular Biology 294, 1159-1168.

Schlenkrich, M. J., Brickmann, J., MacKerell Jr., A. D. \& Karplus, M. (1996). An empirical potential energy function for phospholipids: criteria for parameters optimization and applications. In Biological Membranes. A Molecular Perspective from Computation and Experiment (ed. K. M. Merz \& B. Roux), pp. 31-81. Boston: Birkhauser.

Schumaker, M. F., Pomes, R. \& Roux, B. (2000). A combined molecular dynamics and diffusion model of single proton conduction through gramicidin. Biophysical Journal 79, 2840-2857.

Schumaker, M. F., Pomes, R. \& Roux, B. (2001). Framework model for single proton conduction through gramicidin. Biophysical Journal 80, 12-30.

Schuss, Z., Nadler, B. \& Eisenberg, R. S. (2001). Derivation of Poisson and Nernst-Planck equations in a bath and channel from a molecular model. Phys Rev (E) 64, 036116.

SchutZ, C. N. \& Warshel, A. (2001). What are the dielectric constants of proteins and how to validate electrostatic models? Protein: Structure, Function, Genetics 44, 400-417.

Sharp, K. A. \& Honig, B. (1995). Salt effects on nucleic acids. Current Opinion in Structural Biology 5, 323-328.

Shrivastava, I. H. \& SAnsom, M. S. (2000). Simulations of ion permeation through a potassium channel: molecular dynamics of KcsA in a phospholipid bilayer. Biophysical Journal 78, 557-570.

Sigworth, F. J. (1993). Voltage gating of ion channels. Quarterly Reviews of Biophysics 27, 1-40.

Simonson, T. (2003). Electrostatics and dynamics of proteins. Report of Progress in Physics 66, 737-787.

Sitkoff, D., Sharp, K. A. \& Honig, B. (1994). Accurate calculation of hydration free energies using macroscopic solvent models. Journal of Physical Chemistry 98, 1978.

Siva, K. \& Elber, R. (2003). Ion permeation through the gramicidin channel: atomically detailed modeling by the Stochastic Difference Equation. Proteins 50, 63-80.

Smart, O.S., Goodfellow, J. M. \& Wallace, B. A. (1993). The pore dimension of gramicidin A. Biophysical Journal 65, 2455-2460.

Smith, G. R. \& SAnsom, M. S. (1999). Effective diffusion coefficients of $\mathrm{K}^{+}$and $\mathrm{Cl}^{-}$ions in ion channel models. Biophysical Chemistry 79, 129-151.

Smith, G. R. \& Sansom, M. S. (2002). Free energy of a potassium ion in a model of the channel formed by an amphipathic leucine-serine peptide. European Biophysical Journal 31, 198-206.

Smith, R., Thomas, D. E., Atkins, A. R., Separovic, F. \& Cornelu, B. A. (1990a). Solid-state ${ }^{13} \mathrm{C}-\mathrm{NMR}$ studies of the effects of sodium ions on the gramicidin a ion channel. Biochimica et Biophysica Acta 1026, 161-166. 
Smith, R., Thomas, D. E., Atkins, A. R., Separovic, F. \& Cornell, B. A. (1990b). Solid-state ${ }^{13} \mathrm{C}-\mathrm{NMR}$ studies of the effects of sodium ions on the gramicidin a ion channel. Biocbimica et Biophysica Acta 1026, 161-166.

Smoluchowski, M. V. (1916). Drei Vorträge Über Diffusion, Brownsche Molekularbewegung und Koagulation von Kolloidteilchen. Physikalische Zeitschrift 17, $557-571$.

Soares, C. M., BJorksten, J. \& TApia, O. (1995). L3 loopmediated mechanisms of pore closing in porin: a molecular dynamics perturbation approach. Protein Engineering 8, 5-12.

Stephan, W., Kleutsch, B. \& Frehland, E. (1983). Rate theory models for ion transport through rigid pores. III. Continuum vs discrete models in single file diffusion. Journal of Theoretical Biology 105, 287-310.

Stern, H. A. \& Feller, S. E. (2003). Calculation of the dielectric permittivity profile of a nonuniform system: application to a lipid bilayer simulation. Journal of Chemical Physics 118, 3401-3412.

Straub, J. E., Berne, B. J. \& Roux, B. (1990). Spatialdependent friction for pair diffusion in a simple fluid. Journal of Physical Chemistry 93, 6804-6812.

Suenaga, A., Komeij, Y., Uebayasi, M., Meguro, T., Saito, M. \& Yamato, I. (1998). Computational observation of an ion permeation through a channel protein. Bioscience Report 18, 39-48.

TANG, P. \& Xu, Y. (2002). Large-scale molecular dynamics simulations of general anesthetic effects on the ion channel in the fully hydrated membrane: the implication of molecular mechanisms of general anesthesia. Proceedings of the National Academy of Sciences USA 99, 16035-16040.

Thompson, A. P., Ford, D. M. \& Heffelfinger, G. S. (1998). Direct molecular simulation of gradient-driven diffusion. Journal of Chemical Physics 109, 6406-6414.

Thompson, A. P. \& Heffelfinger, G. S. (1999). Direct molecular simulation of gradient-driven diffusion of large molecules using constant pressure. Journal of Chemical Physics 110, 10693-10705.

Tian, F., Lee, K. C., Hu, W. \& Cross, T. A. (1996). Monovalent cation transport: lack of structural deformation upon cation binding. Biochemistry 35, 11959-11966.

Tieleman, D. P., Berendenen, H. J. \& Sansom, M. S. (2001a). Voltage-dependent insertion of alamethicin at phospholipid/water and octane/water interfaces. Biophysical Journal 80, 331-346.

Tieleman, D. P. \& Berendenen, H. J. C. (1998). A molecular dynamics study of the pores formed by $E$. coli OmpF porin in a fully hydrated POPE bilayer. Biophysical Journal 74, 2786-2801.

Tieleman, D. P., Berendsen, H. J. C. \& Sansom, M. S. P. (1999). An alamethicin channel in a lipid bilayer: molecular dynamics simulations. Biophysical Journal 76, $1757-1769$.
Tieleman, D. P., Biggin, P. C., Smith, G. R. \& Sansom, M. S. (2001b). Simulation approaches to ion channel structure-function relationships. Quarterly Reviews of Biophysics 34, 473-561.

Tieleman, D. P., Forrest, L. R., Sansom, M. S. \& Berendsen, H. J. (1998). Lipid properties and the orientation of aromatic residues in $\mathrm{OmpF}$, influenza M2, and alamethicin systems: molecular dynamics simulations. Biochemistry 37, 17554-17561.

Tobias, D. J. \& Brooks III., C. L. (1987). Calculation of free energy surface using the methods of thermodynamic perturbation theory. Chemical Physics Letters 142, 472-476.

Torrie, G. M. \& Valleau, J. P. (1974). Monte Carlo Free Energy Estimates Using Non-Boltzmann Sampling: application to the sub-critical Lennard-Jones fluid. Chemical Physics Letters 28, 578-581.

Torrie, G. M., Kusalik, P. G. \& Patey, G. N. (1988). Molecular-solvent model for an electrical doublelayer-reference hypernetted-chain (RHNC) results for solvent structure at a charged surface. Journal of Chemical Physics 88, 7826-7840.

Townsley, L. E., Tucker, W. A., Sham, S. \& Hinton, J. F. (2001). Structures of gramicidins A, B, and C incorporated into sodium dodecyl sulfate micelles. Biochemistry 40, 11676-11686.

Urry, D. W. (1971). The gramicidin A transmembrane channel: a proposed $\pi_{L D}$ helix. Proceedings of the National Academy of Sciences USA 68, 672-676.

Urry, D. W., Prasad, K. U. \& Trapane, T. L. (1982). Location of monovalent cation binding sites in the gramicidin channel. Proceedings of the National Academy of Sciences USA 79, 390-394.

Valleau, J. P. \& Torrie, G. M. (1977). A guide for Monte Carlo for statistical mechanics. In Statistical Mechanics, Part A (ed. Berne), pp. 169-194. New York: Plenum Press.

van Dujneveldt, S. \& Frenkel, D. (1992). Computersimulation study of free-energy barriers in crystal nucleation. Journal of Chemical Physics 96, 4655-4668.

van Gunsteren, W. F., Daura, X. \& Mark, A. E. (1999). GROMOS force field. In Encyclopaedia of Computational Chemistry, vol. 2 (editor-in-chief P. von Ragu Schelyer), pp. 1211-1216. New York: John Wiley \& Sons.

Walz, D., Bamberg, E. \& Lauger, P. (1969). Nonlinear electrical effects in lipid bilayer membranes. I. Ion injection. Biophysical Journal 9, 1150-1159.

Wang, J., Kim, S., Kovacs, F. \& Cross, T. A. (2001). Structure of the transmembrane region of the M2 protein $\mathrm{H}(+)$ channel. Protein Science 10, 2241-2250.

Warshel, A. \& Åqvist, J. (1991). Electrostatic energy and macromolecular function. Annual Review of Biophysics and Biophysical Chemistry 20, 267-298.

Warshel, A. \& Papazyan, A. (1998). Electrostatic effects in macromolecules: fundamental concepts and practical modeling. Current Opinion in Structural Biology 8, 211-217. 
Warwicker, J. \& Watson, H. C. (1982a). Calculation of the electric potential in the active site cleft due to alphahelix dipoles. Journal of Molecular Biology 157, 671-679.

WARwicker, J. \& Watson, H. C. (1982b). Calculation of the electric potential in the active site cleft due to alpha-helix dipoles. Journal of Molecular Biology 57, 671-679.

Watanabe, M., Rosenbusch, J., Schirmer, T. \& Karplus, M. (1997). Computer simulations of the OmpF porin from the outer membrane of Escherichia coli. Biophysical Journal 72, 2094-2102.

Weiss, M. S., Abele, U., Weckesser, J., Welte, W., Schiltz, E. \& Schulz, G. E. (1991). Molecular architecture and electrostatic properties of a bacterial porin. Science 254, 1627-1630.

Wood, M. D. \& Friedman, H. L. (1987). Brownian dynamics simulation of models for aqueous $1 \mathrm{M} \mathrm{NaCl}$ and 1/2M CuSO4. Zeitschrift für Physikalische Chemie Neue Folge Bd. 155, 121-132.

Woolf, T. B. \& Roux, B. (1994a). The conformational flexibility of o-phosphorylcholine and o-phosphorylethanolamine: a molecular dynamics study of solvation effects. Journal of American Chemical Society 116, 5916-5926.

Woolf, T. B. \& Roux, B. (1994b). Molecular dynamics simulation of the gramicidin channel in a phospholipid bilayer. Proceedings of the National Academy of Sciences USA 91, 11631-11635.

Woolf, T. B. \& Roux, B. (1996). Structure, energetics and dynamics of lipid-protein interactions: a molecular dynamics study of the gramicidin A channel in a DMPC bilayer. Protein: Structure, Function Genetics 24, 92-114.

Woolf, T. B. \& Roux, B. (1997). The binding site of sodium in the gramicidin A channel: a comparison of molecular dynamics simulations with solid state NMR data. Biophysical Journal 72, 1930-1945.
Yang, Y., Henderson, D. \& Busath, D. (2003). Appliedfield molecular dynamics study of a model calcium channel selectivity filter. Journal of Chemical Physics (B) 118, 4213-4220.

Zachariae, U., Helms, V. \& Engelhardt, H. (2003). Multistep mechanism of chloride translocation in a strongly anion-selective porin channel. Biophysical Journal 85, 954-962.

Zachariae, U., Koumanov, A., Engelhardt, H. \& Karshikoff, A. (2002). Electrostatic properties of the anion selective porin Omp32 from Delftia acidovorans and of the arginine cluster of bacterial porins. Protein Science 11, 1309-1319.

Zhong, Q., Newns, D. M., Pattnaik, P., Lear, J. D. \& KLeIN, M. L. (2000). Two possible conducting states of the influenza A virus M2 ion channel. FEBS Letters 473, 195-198.

Zhou, M., Morais-Cabral, J. H., Mann, S. \& MacKinnon, R. (2001a). Potassium channel receptor site for the inactivation gate and quaternary amine inhibitors. Nature 411, 657-661.

Zhou, Y., Morais-Cabral, J.H., Kaufman, A. \& MacKinnon, R. (2001b). Chemistry of ion coordination and hydration revealed by a $\mathrm{K}^{+}$channel-Fab complex at 2.0 A resolution. Nature 414, 43-48.

ZWANZIG, R. W. (1954). High temperature equation of state by a perturbation method. Journal of Chemical Physics 22, 1420-1426.

Zwanzig, R. W. (1961). In Lectures in Theoretical Physics, vol. III (eds. W. E. Britton, B. W. Downs \& J. Down), p. 135. New York: Interscience.

Zwanzig, R. W. (1965). Time-correlation functions and transport coefficients in statistical mechanics. Annual Review of Physical Chemistry 16, 67-102.

Zwanzig, R. W. (2001). Nonequilibrium Statistical Mechanics. New York, Oxford: Oxford University Press. 